\title{
Status of the Development of an Embedded Transport Treatment of Control Rods and of Radial Flux Expansion in Cylindrical Nodal Diffusion Codes
}

Frederick N. Gleicher II Abderrafi M. Ougouag

September 2009
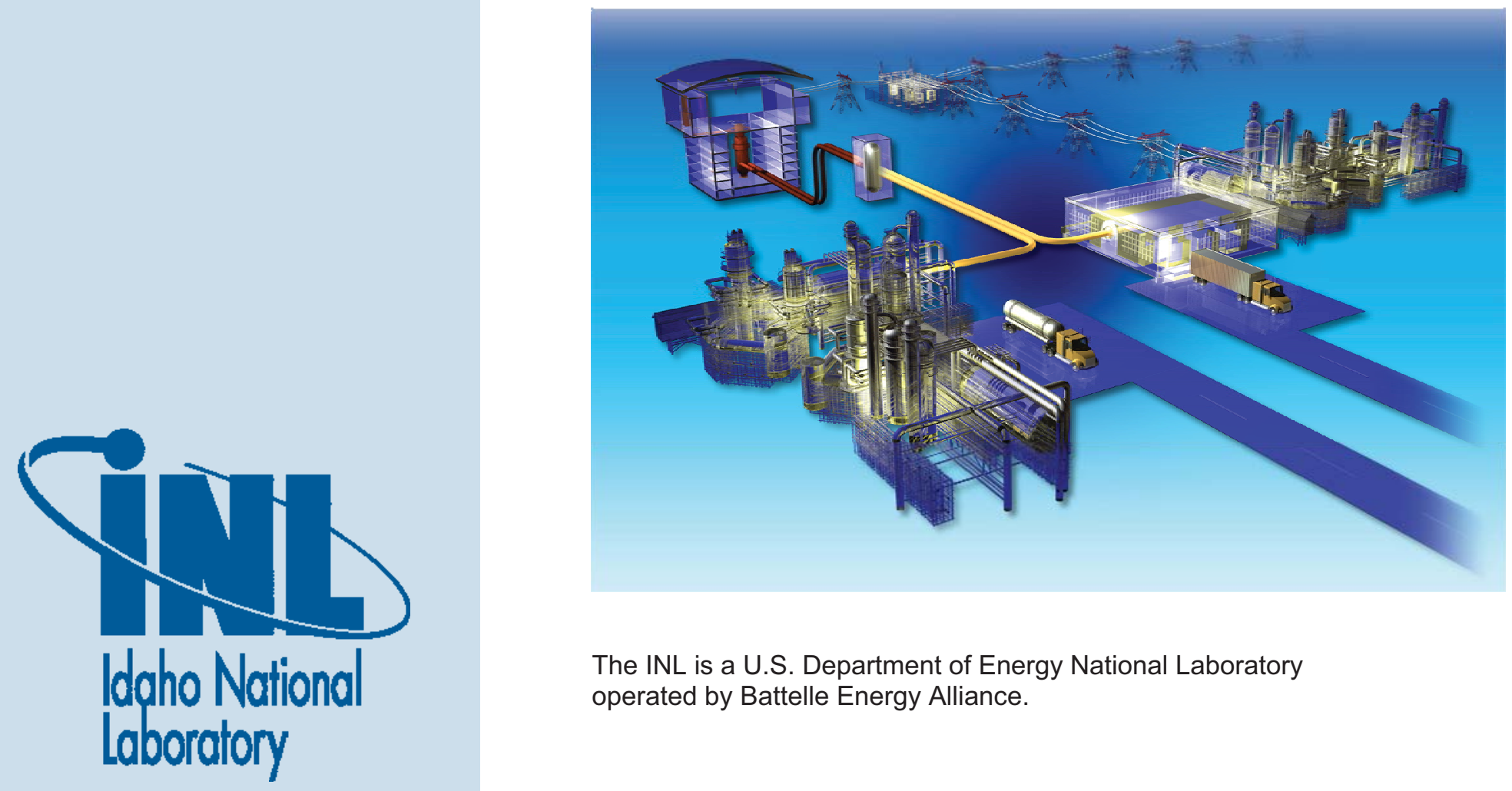

The INL is a U.S. Department of Energy National Laboratory operated by Battelle Energy Alliance. 


\section{DISCLAIMER}

This information was prepared as an account of work sponsored by an agency of the U.S. Government. Neither the U.S. Government nor any agency thereof, nor any of their employees, makes any warranty, expressed or implied, or assumes any legal liability or responsibility for the accuracy, completeness, or usefulness, of any information, apparatus, product, or process disclosed, or represents that its use would not infringe privately owned rights. References herein to any specific commercial product, process, or service by trade name, trade mark, manufacturer, or otherwise, does not necessarily constitute or imply its endorsement, recommendation, or favoring by the U.S. Government or any agency thereof. The views and opinions of authors expressed herein do not necessarily state or reflect those of the U.S. Government or any agency thereof. 


\section{Status of the Development of an Embedded Transport Treatment of Control Rods and of Radial Flux Expansion in Cylindrical Nodal Diffusion Codes}

Frederick N. Gleicher II, Abderrafi M. Ougouag

September 2009

Idaho National Laboratory

Next Generation Nuclear Plant Project

Idaho Falls, Idaho 83415

Prepared for the

U.S. Department of Energy

Office of Nuclear Energy

Under DOE Idaho Operations Office

Contract DE-AC07-05ID14517 



\title{
Next Generation Nuclear Plant Project
}

\section{Status of the Development of an Embedded Transport Treatment of Control Rods and of Radial Flux Expansion in Cylindrical Nodal Diffusion Codes}

\author{
INL/EXT-09-16788
}

September 2009

Approved by:
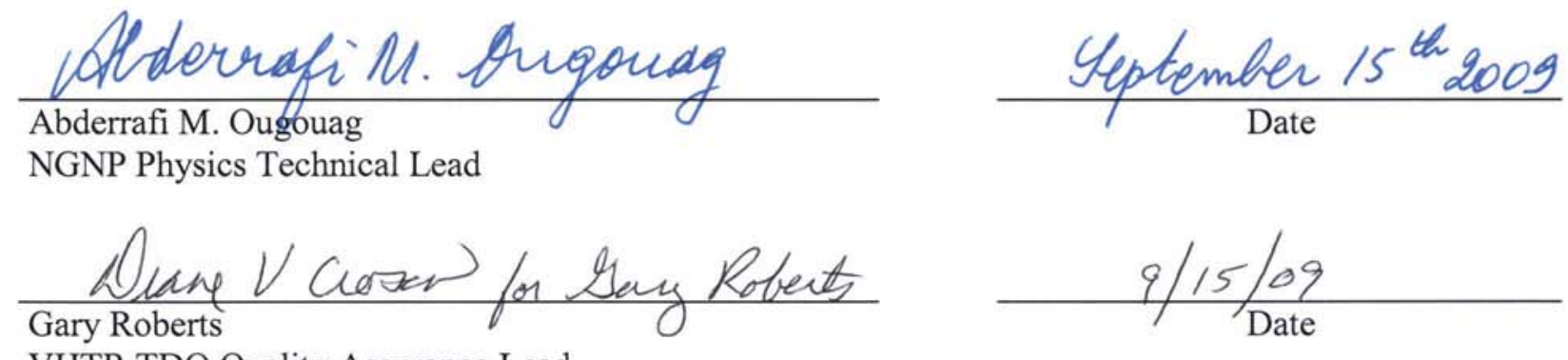

VHTR TDO Quality Assurance Lead
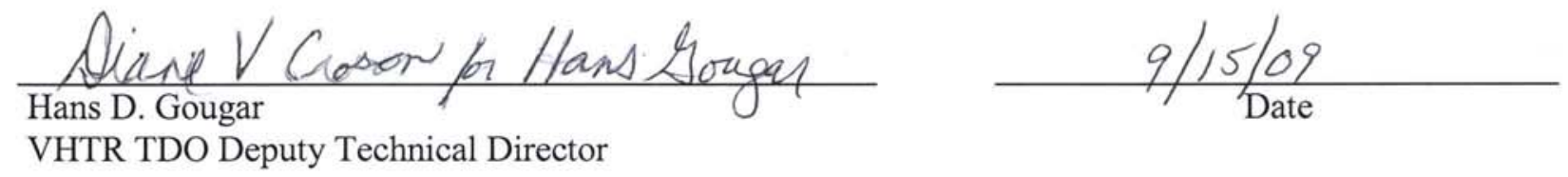



\begin{abstract}
A new diffusion-transport hybrid nodal method in $\mathrm{R}-\mathrm{Z}$ is presented that can effectively treat non-multiplying zones in pebble bed reactors. The new method seamlessly combines the analytic coarse mesh finite difference (CMFD) diffusion formulation and a transport theory based response matrix formulation while retaining the properties and structure of the CMFD diffusion solver. The resulting combined formulation is utilized in selected non-multiplying nodes to capture angular effects on the flux. Test results indicate that the method has been implemented correctly into the CYNOD reactor kinetics code.

This document also presents a status report on the development of a better source approximation for the Green's function nodal solution in the radial direction of cylindrical geometry. The basic theory has been developed, including obtaining polynomials that are orthonormal over the domain of integration and the derivation of approximately half of the required matrix elements (single and double integrals in the source expansions).
\end{abstract}




\section{ACKNOWLEDGEMENTS}

Work supported by the U.S. Department of Energy, Assistant Secretary for the office of Nuclear Energy, under DOE Idaho Operations Office Contract DEAC07-05ID14517. 


\section{CONTENTS}

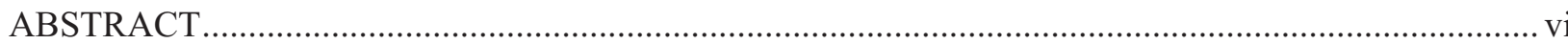

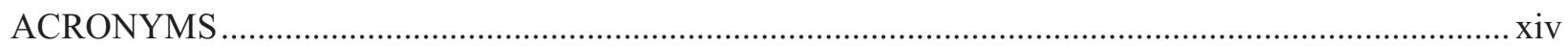

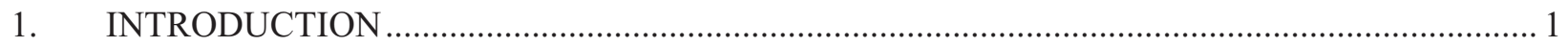

2. OVERVIEW OF METHODS FOR TREATING CONTROL RODS IN HTRS ............................. 2

3. THE AVERAGED NODAL RESPONSE BALANCE EQUATION …....................................... 4

4. DERIVATION OF THE COMMON AXIAL INTERFACE NET CURRENT

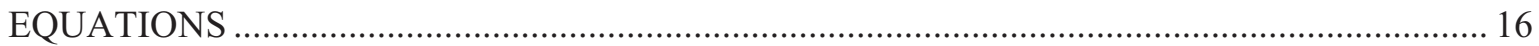

4.1 Common Interface Current for Two Response Nodes Adjoining an Axial Boundary........... 16

4.2 Common Interface Current for a Response Node Adjoining a Diffusion Node on the

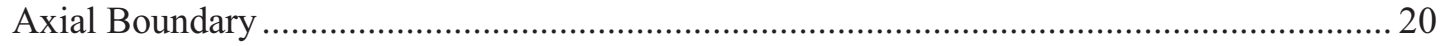

4.3 Common Interface Current for a Response Node Adjoining an Axial Boundary................. 24

5. COMMON RADIAL INTERFACE NET CURRENT EQUATIONS............................................28

5.1 Common Interface Current for Two Response Nodes With an Adjoining Boundary

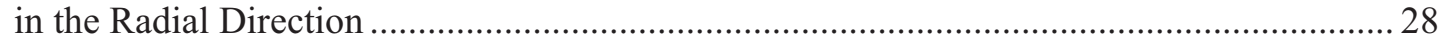

5.2 Common Interface Current for a Response Node Adjoining a Diffusion Node on the

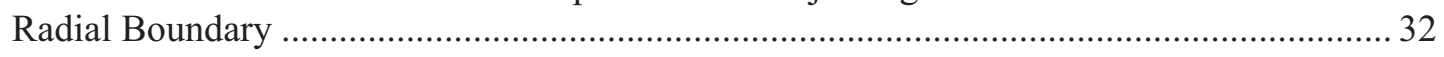

5.3 Common Interface Current for a Response Node Adjoining a Radial Boundary .................. 36

6. EMBEDDING THE RESPONSE FUNCTION NODES INTO THE MATRIX FOR THE

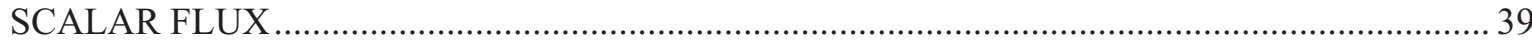

7. COMPUTATIONAL TESTS AND VERIFICATION OF METHOD

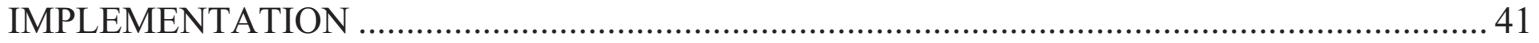

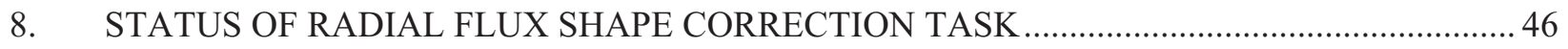

8.1 Solution for the Transverse-Integrated Radial Nodal Flux ............................................... 46

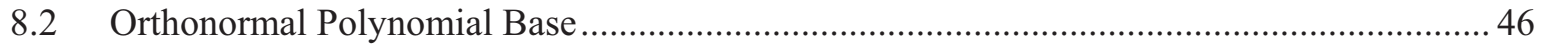

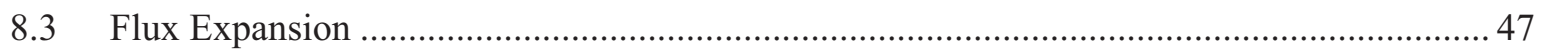

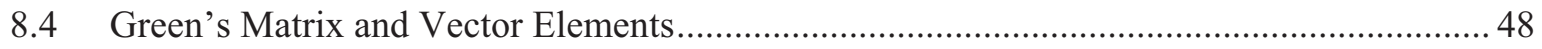

8.4.1 Identification of Required Integrals ........................................................................ 48

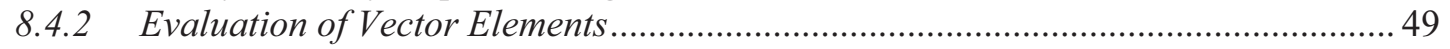

8.4.3 Compact form for the Vector Elements .................................................................... 51

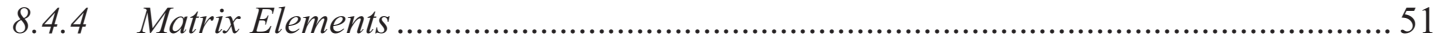

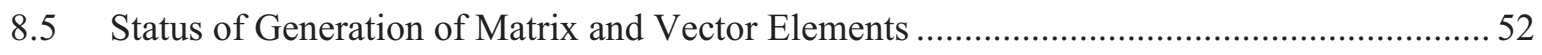

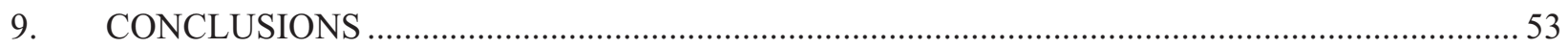

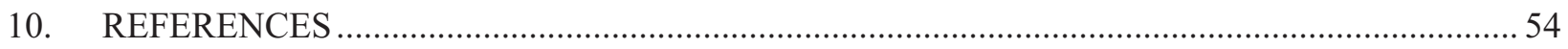




\section{FIGURES}

Figure 1. Test model with partially inserted control rods in top portion of reactor................................... 42

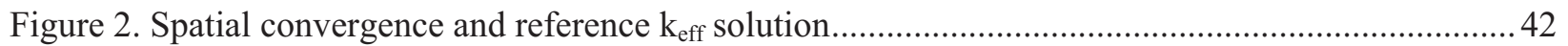

Figure 3 Test model with partially inserted control rods in bottom portion of reactor..............................43

Figure 4. Test model with fully inserted curtain control rods................................................................... 44 


\section{TABLES}

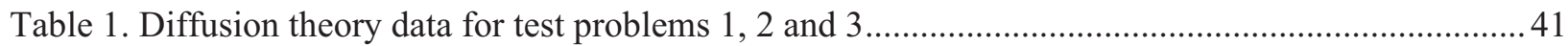

Table 2. Testing matrix for the hybrid method (partially inserted control rods from top)........................43

Table 3. Testing matrix for the hybrid method (partially inserted control rods from bottom) ..................43

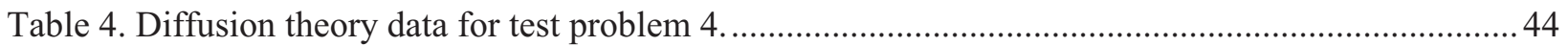

Table 5. Testing matrix for the hybrid method (fully inserted control rods). .......................................... 44 


\section{ACRONYMS}

CYNOD

CMFD

HTR

INL

LWR

MECS

NGNP

PBMR

VSOP
CYlindrical NODal code

Coarse Mesh Finite Difference

High Temperature Reactor

Idaho National Laboratory

Light Water Reactor

Method of Equivalent Cross Sections

Next Generation Nuclear Plant

Pebble Bed Modular Reactor

Very Superior Old Programs 


\section{Status of the Development of an Embedded Transport Treatment of Control Rods and of Radial Flux Expansion in Cylindrical Nodal Diffusion Codes}

\section{INTRODUCTION}

Accurate knowledge of the neutron as a function of space and time is essential to the correct modeling of the behavior and safety of nuclear reactors. Diffusion theory yields a good approximation to a neutron field when scattering dominates within the medium under consideration, i.e., when the medium is predominantly diffusive, and when the scattering is isotropic or only weakly anisotropic. In regions with strong neutron absorbers these conditions are not satisfied. In particular, the neutron field depends highly on angle and the approximation of diffusion breaks down. Yet, diffusion theory solvers that rely on nodal methods have been successfully developed for light water reactor (LWR) analysis because the transport effects of these absorbers are captured in the auxiliary process of homogenization that uses detailed single-node (assembly) transport calculations. These assembly homogenization calculations yield effective nodal diffusion parameters and discontinuity factors that allow the (mild) transport effects of LWR physics to be captured in full core diffusion simulations. This approach yields very accurate corewide neutron flux distributions using a computationally efficient algorithm.

For historical reasons, application of the nodal method approach to high temperature rectors (HTR) has lagged. This is in part the consequence of an absence of commercial development or deployment of this type of reactor concept over the past two decades. The peculiarities of HTRs (long mean free path, cylindrical or hexagonal geometry, and moving fuel in the case of the pebble bed reactor) are not adequately treated by existing LWR analysis tools. The INL is developing the PEBBED and CYNOD codes for nodal static (including depletion) and kinetic analysis of pebble bed reactors. The new codes bring a modern nodal formulation to the design and safety analysis of the NGNP. However, the development of cylindrical geometry nodal methods for the modeling of the pebble bed HTR presents significant technical challenges. One of these challenges is the correct treatment of control rods embedded in reflectors, i.e., in nonmultiplying regions, which contrasts with the placement of control rods within or adjacent to fueled zones in LWRs. The placement of control rods in nonmultiplying zones renders the application of modern equivalence theory inappropriate for the preparation of homogenized diffusion theory data and the related discontinuity factors. This challenge is addressed in this work through the use of an embedded transport theory treatment seamlessly within the nodal diffusion code.

In Section 2 a succinct, noncomprehensive, survey of methods used for the treatment of control rods in HTRs is presented and the motivation for the method proposed in this report is given. Section 3 summarizes the derivation of the balance equation for a given response node. A node is termed a "response node" when it is treated using the response function approach as opposed to the nodal diffusion approach. In Section 4, starting from response function, equations are derived that express interface net currents for the axial direction for nodes near a boundary. The net current expressions are subsequently incorporated into the nodal balance equation in order to produce a coarse mesh finite difference (CMFD) scheme that seamlessly mixes diffusion and transport solutions. In Section 5 the equations for the net current on radial nodal interfaces are derived for combinations of inner and outer nodes. Section 6 discusses the formation of the three-stripe or five-stripe matrix for the scalar (diffusion-equivalent) flux. The matrix seamlessly incorporates diffusion treatment in some nodes and transport treatment in others. In Section 7 preliminary results are given that test the implementation. Section 8 presents a summary of the progress on the development of radial source expansions, and Section 9 presents some conclusions. Section 10 lists the previous works cited in the report. 


\section{OVERVIEW OF METHODS FOR TREATING CONTROL RODS IN HTRS}

The modeling of control rods in HTRs has been approached in various ways with varying degrees of success. One approach is the Method of Equivalent Cross Sections (MECS)used in the VSOP code that was evaluated by the Pebble Bed Modular Reactor (PBMR) project. ${ }^{1}$ In the MECS, a fine mesh transport solution is obtained for the heterogeneous region of interest, and the reaction rates of interest are computed. The region of interest is often known as a super cell. Calculated leakage rates of the fine mesh transport are matched to the calculated leakage rates of the neighboring nodes where the neighboring nodes are treated with diffusion. An equivalent average flux for the super cell is calculated based on the average cell flux and edge cell flux values of the neighboring diffusion treated nodes. The equivalent cross sections are calculated from the super cell average flux and the reaction rates. This is done in the MECS through analyst intervention - careful specification of the thickness of the super cell—such that the leakage rates of the transport solution are equivalent to those of an auxiliary diffusion solution.

Transport and diffusion leakage rates must match at the edges of the super cell. Since the control rod is often at the center of the super cell, the resulting restrictions on the location and size of the absorber node that can be inconsistent with the nodalization of the rest of the model and lead to some analytical uncertainty. Overall, this method requires considerable engineering judgment (in other words, analytical iterative analyst intervention) and is thus sensitive to core design and prone to uncertainty. Nonetheless, the MECS approach has been validated for a configuration of the Russian ASTRA critical facility as part of the overall validation of the VSOP code used in PBMR core design. ${ }^{1}$

A second method for the treatment of control rods is the use of modified boundary conditions with response functions. ${ }^{2}$ In traditional whole core simulation, the coupling between nodes is achieved using coupling coefficients derived from the diffusion theory parameters generated in an assembly transport calculation. Such calculations are difficult to perform for reflector nodes because of the lack of a neutron source. Usually a small amount of fuel is artificially added to the reflector composition to generate the neutron flux, which in reality streams in from adjacent nodes. This approach yields spatial and spectral errors in the nodal flux. In Fen et al.'s approach, response functions are generated from the assembly calculation and used as modified boundary conditions in the whole core diffusion calculation. Response functions relate the normalized partial current impinging on one face of a node to the currents leaving that face and the other, remaining, faces. Like the normal diffusion parameters, response functions can be generated from assembly transport calculations but they do not require an internal source and they directly account for the spatial asymmetry of internodal currents.

The response function method avoids having to choose possibly thick regions to capture equivalent leakages when control rods are present. The Fen method does not, however, obtain the averaged scalar flux for the internal boundary regions and does not obtain the net currents inside internal boundary regions. Furthermore, should a response region need to be changed or extended, the analyst must rerun the entire response calculation to obtain new boundary conditions.

In this report a new method is proposed where response functions are used to model the control rod region. The method differs significantly from the second method discussed above by incorporating the response functions directly as an embedded scheme within the nodal formulation; the response functions are used to generate coupling coefficients morphologically identical to the diffusion-theory-based coefficients they replace. Different types of response functions are required depending on whether the node is an interior node in the whole core model or a single boundary node, or a node belonging to a continuous set of nodes, one of which is a boundary node. The response functions for the different node types and compositions can be computed using the usual assembly calculations. 
The embedded formulation has significant advantages over the previous methods. By formulating the response functions into a nodal form consistent with the existing whole core diffusion model, the average node flux is obtained even in the response function region. Also, should the analyst have the appropriate response functions available and wish to extend the response function region, the analyst just needs to change the nodes to the correct type. A whole new response function does not need to be generated with this method.

Another advantage is that thick regions do not have to be prescribed for the treatment of control rods. There is no requirement that internodal leakage values of the assembly transport calculation match those of the whole core diffusion calculation. Instead, responses are generated based upon the incoming currents of a response region. This response node is allowed to be of any thickness, and the incoming partial currents, outgoing partial currents, and fluxes are calculated directly by the new hybrid method. This embedded nodal formulation has been shown to work in 1-D in the axial direction, and the purpose of this report is to present the extension of this technique to 2-D R-Z geometry. In the 1-D work, the response functions were incorporated into a highly accurate Green's function-based nodal implementation, ${ }^{3}$ and for the 2-D case, the embedded nodal formulation is incorporated into a highly accurate R-Z Green's function nodal code. The R-Z Green's function method is well documented elsewhere ${ }^{3}$ and will not be discussed in detail in this report. Instead, the hybridization - the direct incorporation of response functions into the nodal solver-will be discussed in detail. 


\section{THE AVERAGED NODAL RESPONSE BALANCE EQUATION}

The nodal balance equation for nodes with the response function treatment is derived in this section. The nodal balance equation is the foundation from which the CMFD form of nodal diffusion solvers is constructed. An equivalent equation, based on response functions, is the foundation for the transformation of response function-based neutron conservation laws into the CMFD form. Therefore, the response nodal balance equation is the starting point for the formation of either the three- or the five-stripe row of the global coupling matrix for the average nodal scalar fluxes. The equations for the interface currents involve the average scalar flux of two adjacent nodes, sources within said nodes, and transverse leakage currents into or out of the nodes. To form the matrix of the scalar flux, the expressions for the common interface currents are substituted into the appropriate nodal balance equation for the node. For a matrix row that is centered on a node with the response function treatment, the balance equation that is used is the response balance equation.

Another use of the response balance equation is as a means to eliminate selected net currents. The net currents opposite of a common interface are eliminated by substituting an expression for that particular net current derived from the balance equation. The result is an equation for the common interface net current that contains the average fluxes of the two adjacent nodes, source terms within those nodes, and net transverse leakage terms into those nodes. This equation is free of any net currents on the opposite faces of the common boundary.

The derivation of the response balance equation involves obtaining the outgoing currents (exiting partial currents) either numerically or analytically as responses to the incoming currents for the node. This is done using the response function treatment. From the response equations that express the outgoing currents, a set of equations for the net currents is obtained. These equations are then manipulated to obtain a set of equations in which the incoming currents are expressed in terms of the net currents. The equations for the incoming currents are then substituted into the response equation for the average scalar flux.

The derivation for a response node starts with the formulation of the exiting currents as responses to the incoming currents. The equations for the exiting currents for a 2-D r-z response node are

$$
\begin{aligned}
& J_{i, k}^{\text {out }, g, r_{a}}=\sum_{g^{\prime}=1}^{G} \sum_{f=r_{a}, r_{b} \cdot a_{z^{\prime}} a_{-z}} R_{g^{\prime} \rightarrow g, f \rightarrow r_{a}}^{J_{i, k}^{\text {out, }, r_{a}}} J_{i, k}^{i n, g^{\prime}, f} \\
& J_{i, k}^{\text {out }, g, \boldsymbol{r}_{b}}=\sum_{g^{\prime}=1}^{G} \sum_{f=\boldsymbol{r}_{a^{\prime}}, \boldsymbol{r}_{b^{\prime}}, a_{z^{\prime}}, a_{-z}} R_{g \rightarrow g, f \rightarrow \boldsymbol{r}_{b}}^{J_{i, k}^{\text {out, , , } r_{b}}} J_{i, k}^{i n, g^{\prime}, f} \\
& J_{i, k}^{\text {out }, g, a_{z}}=\sum_{g^{\prime}=1}^{G} \sum_{f=r_{a^{\prime}} r_{b^{\prime}} \cdot a_{z}, a_{-z}} R_{g^{\prime} \rightarrow g, f \rightarrow a_{z}}^{J_{i, k}^{\text {out }, g, a_{z}}} J_{i, k}^{i n, g^{\prime}, f}
\end{aligned}
$$

and

$$
J_{i, k}^{\text {out }, g, a_{-z}}=\sum_{g^{\prime}=1}^{G} \sum_{f=r_{a^{\prime}}, r_{b^{\prime}} \boldsymbol{a}_{z^{\prime}} \boldsymbol{a}_{-z}} R_{g^{\prime} \rightarrow g, f \rightarrow a_{-z}}^{J_{i, k}^{\text {out }, g, a_{-z}}} J_{i, k}^{i n, g^{\prime}, f}
$$

In Equations (1), (2), (3), and (4) the exiting current is determined from the response of the node to an incoming current and the left, right, top, and bottom edges of the response node are labeled as $r_{a}, r_{b}, a_{z}$, and $-\mathrm{a}_{\mathrm{z}}$, respectively. The energy group is shown by the index $\mathrm{g}$. The upper symbol (symbol for a current) on 
the response function $(R)$ indicates the original response. The arrow indicates either the response path from a given energy group to an outgoing energy group or a boundary face to the response boundary face response ( $f$, the dummy summation index refers to a face). The exiting current for a face is determined by the summation of responses from all incoming currents from all faces over all contributing energy groups. The exiting currents in Equations (1), (2), (3), and (4) can also be expressed as

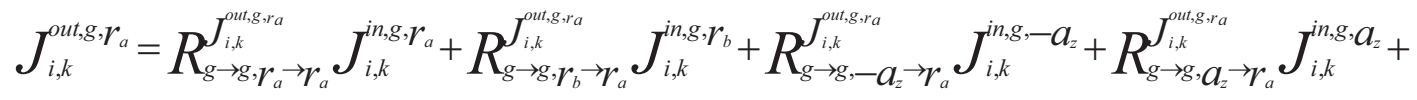

$$
\begin{aligned}
& \sum_{g^{\prime}=1, g^{\prime} \neq g}^{G} \sum_{f=r_{a}, r_{b} \cdot a_{z} a_{-z}} R_{g^{\prime} \rightarrow g, f \rightarrow r_{a}}^{J_{i, k, g, r_{a}}^{\text {on }}} J_{i, k}^{i n, g^{\prime}, f}
\end{aligned}
$$

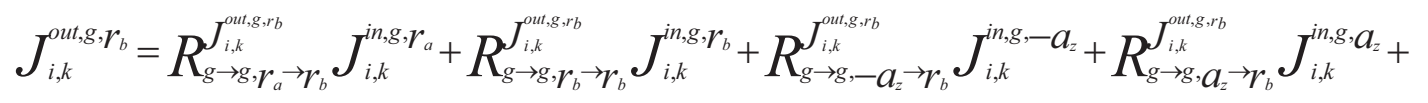

$$
\begin{aligned}
& \sum_{g^{\prime}=1, g^{\prime} \neq g}^{G} \sum_{f=r_{a}, r_{b}{ }^{\prime} a_{z} a_{-z}} R_{g^{\prime} \rightarrow g, f \rightarrow r_{b}}^{J_{i, k, g, r_{b}}^{\text {on }}} J_{i, k}^{i n, g^{\prime}, f}
\end{aligned}
$$

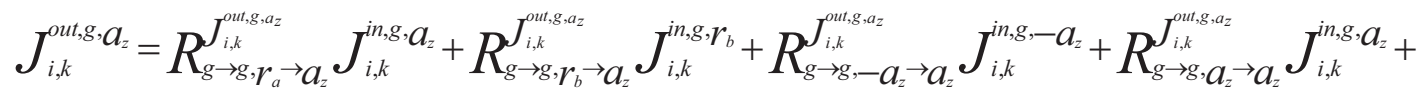

$$
\begin{aligned}
& \sum_{g^{\prime}=1, g^{\prime} \neq g}^{G} \sum_{f=r_{a} r_{b} \cdot a_{z} a_{-z}} R_{g^{\prime} \rightarrow g, f \rightarrow a_{z}}^{J_{i, k, g, a_{z}}^{o n g}} J_{i, k}^{i n, g^{\prime}, f}
\end{aligned}
$$

and

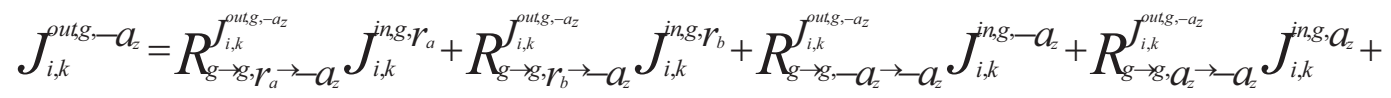

$$
\begin{aligned}
& \sum_{g^{\prime}=1, g^{\prime} \neq g}^{G} \sum_{f=r_{d}, r_{b} a_{z} \cdot a_{z}} R_{g^{\prime} \rightarrow g, f \rightarrow-a_{z}}^{J_{i, k, g,-a_{z}}} J_{i, k}^{i n, g^{\prime}, f}
\end{aligned}
$$

The source terms for the exiting current equations are defined as

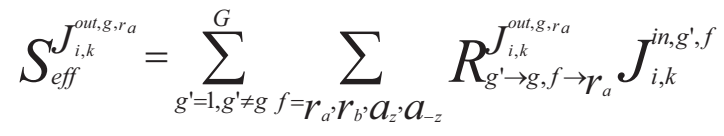

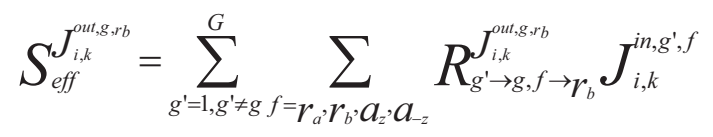

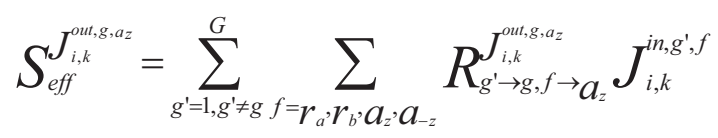

and

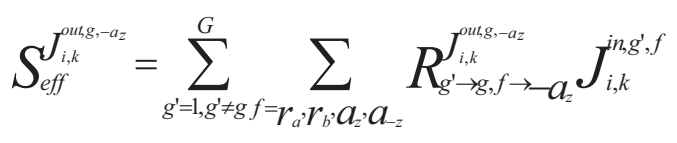


Equations (9), (10), (11), and (12) are the effective sources for the response node (i,k) and are held constant for energy group g. Equations (9), (10), (11), and (12) are substituted into Equations (5), (6), (7), and (8) respectively to obtain

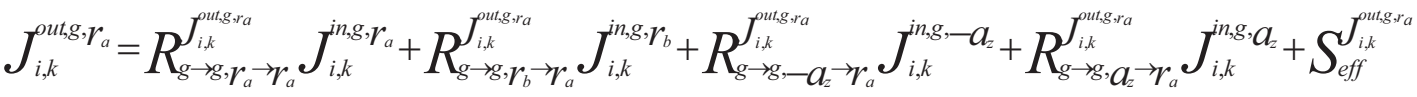

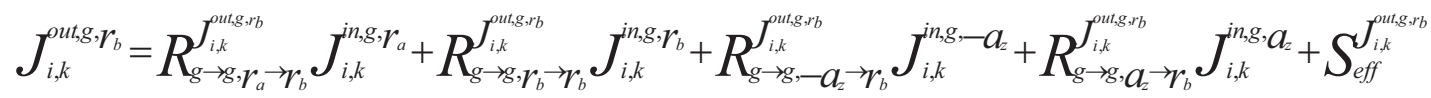

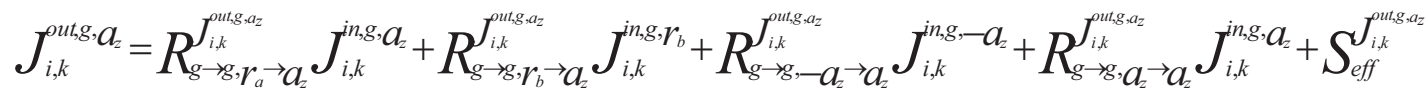

and

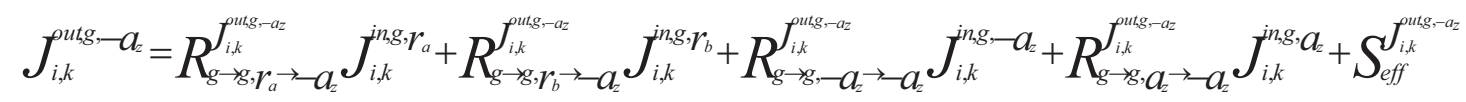

To obtain equations relating the incoming current to the net current, the exiting current in Equations (13), (14), (15), and (16) must be replaced with an expression containing the net current and the incoming current. At each response node edge, the net current can be defined as a combination of incoming and exiting current. The equations relating the side net current to the node incoming and exiting currents are

$$
\begin{aligned}
& J_{i, k}^{\text {netg, }, \boldsymbol{r}_{a}}=-J_{i, k}^{\text {outg, } \boldsymbol{r}_{a}}+J_{i, k}^{i n, g, \boldsymbol{r}_{a}} \\
& J_{i, k}^{\text {net,g, } \boldsymbol{r}_{b}}=J_{i, k}^{\text {out,g, } \boldsymbol{r}_{b}}-J_{i, k}^{i n, g, \boldsymbol{r}_{b}} \\
& J_{i, k}^{\text {net, }, \boldsymbol{a}_{z}}=J_{i, k}^{\text {out, }, \boldsymbol{a}_{z}}-J_{i, k}^{i n, g, \boldsymbol{a}_{z}}
\end{aligned}
$$

and

$$
J_{i, k}^{\text {netg, }-a_{2}}=-J_{i, k}^{\text {outg,- }-a_{2}}+J_{i, k}^{i, g, r_{a}}
$$

The outward currents are obtained from Equations (17), (18), (19), and (20), and then substituted into Equations (13), (14), (15), and (16), respectively. The terms with incoming currents are collected together to obtain

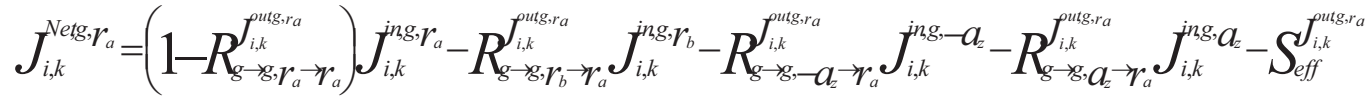

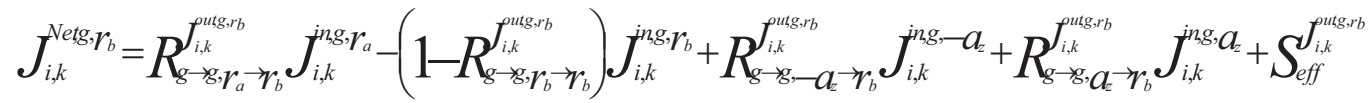

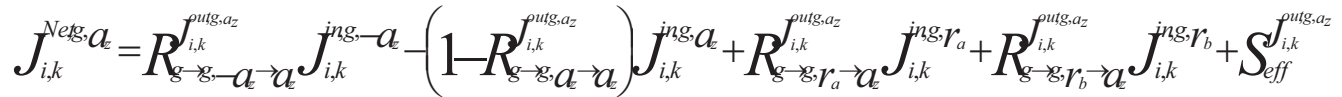

and 


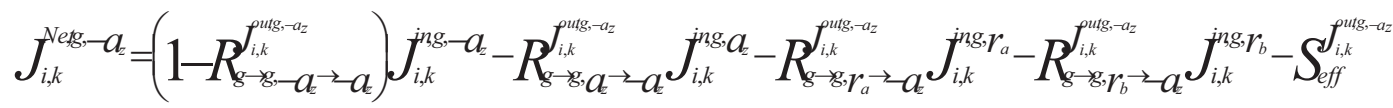

Equations (21), (22), (23), and (24) express the net currents of a response node as a sum of the incoming currents multiplied by coefficients composed of response functions. But the equation for the average scalar flux of a response node is expressed as a sum of the response functions multiplied by the incoming currents. Thus, Equations (21), (22), (23), and (24) must be algebraically manipulated to yield the incoming currents as a sum of the net currents multiplied by coefficients made up of response functions. The incoming currents for the above system of equations can be inverted in multiple ways. Unlike a strictly numerical inversion, an algebraic inversion can be shown to give the correct (exact) balance coefficients when analytical expressions for the response functions are available. In this section, the equations for the axial net currents are manipulated to obtain the axial incoming currents and the equations for the radial net currents are manipulated to obtain the radial incoming currents. Through substitution, the axial incoming currents are eliminated from the radial incoming current equations and the radial incoming currents are eliminated from the axial incoming current equations. The new axial and radial incoming current equations are then inverted per Cramer's rule to obtain a set of equations that express the incoming current as the sum of the net currents of the response node edge multiplied by coefficients composed of response functions.

To obtain an equation with the incoming current on the response node side $r_{b}$, Equation (21) is multiplied by $R_{g \rightarrow g, \boldsymbol{r}_{a} \rightarrow \boldsymbol{r}_{b}}^{J_{i, k, g, r_{b}}}$ and Equation (22) is multiplied by $\left(1-R_{g \rightarrow g, \boldsymbol{r}_{a} \rightarrow \boldsymbol{r}_{a}}^{J_{i, k}^{\text {out }, r_{a}}}\right)$. The two equations are then subtracted and algebraically manipulated to obtain the incoming current on side $r_{b}$. The resulting equation for the incoming current $r_{b}$ is

$$
\begin{aligned}
& J_{i, k}^{i n, g, r_{b}}=-\frac{R_{g \rightarrow g, r_{a} \rightarrow r_{b}}^{J_{i, k, g, r_{b}}^{o n}}}{\bar{R}_{g, r_{a}, r_{a}}} J_{i, k}^{N e t, g, r_{a}}+\frac{\left(1-R_{g \rightarrow g, r_{a} \rightarrow r_{a}}^{J_{i, k}^{o u, g, r_{a}}}\right)}{\bar{R}_{g, \boldsymbol{r}_{a}, \boldsymbol{r}_{a}}} J_{i, k}^{N e t, g, \boldsymbol{r}_{b}} \\
& -\frac{1}{R_{g, r_{a}, r_{a}}}\left[R_{g \rightarrow g, r_{a} \rightarrow r_{b}}^{J_{i, k, g, r_{b}}^{o u n}} R_{g \rightarrow g, a_{z} \rightarrow r_{a}}^{J_{i, k, g, r_{a}}^{o u}}+\left(1-R_{g \rightarrow g, r_{a} \rightarrow r_{a}}^{J_{i, k, r_{a}}^{o u n}}\right) R_{g \rightarrow g, a_{z} \rightarrow r_{b}}^{J_{i, k, g, r_{a}}^{\text {out }}}\right] J_{i, k}^{i n, g, a_{z}}
\end{aligned}
$$

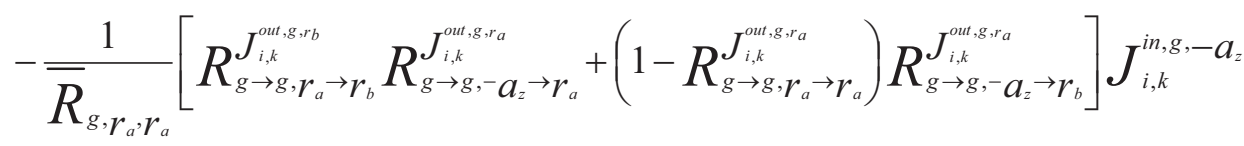

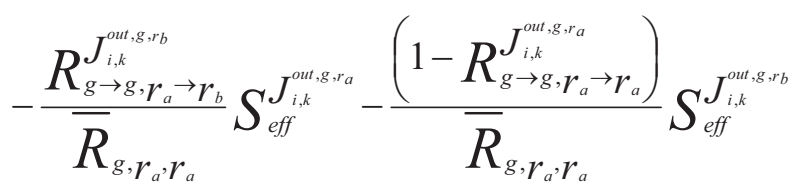

where

$$
\bar{R}_{g, \boldsymbol{r}_{a}, \boldsymbol{r}_{b}}=R_{g \rightarrow g, \boldsymbol{r}_{a} \rightarrow \boldsymbol{r}_{b}}^{J_{i, t, g, r_{b}}^{\text {out }}} R_{g \rightarrow g, \boldsymbol{r}_{b} \rightarrow \boldsymbol{r}_{a}}^{J_{i, t, g, r_{a}}^{\text {out }}}-\left(1-R_{g \rightarrow g, \boldsymbol{r}_{a} \rightarrow \boldsymbol{r}_{a}}^{J_{i, t, g, r_{a}}^{\text {out }}}\right)\left(1-R_{g \rightarrow g, \boldsymbol{r}_{b} \rightarrow \boldsymbol{r}_{b}}^{J_{i, t, g, r_{b}}^{\text {out }}}\right)
$$


To obtain the equation for the incoming current on the response node side $r_{a}$, Equation (21) is

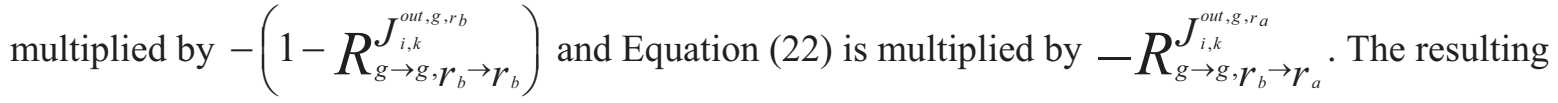
equations are subtracted and algebraically manipulated to obtain the incoming current on side $r_{a}$. The resulting equation for side $r_{a}$ is

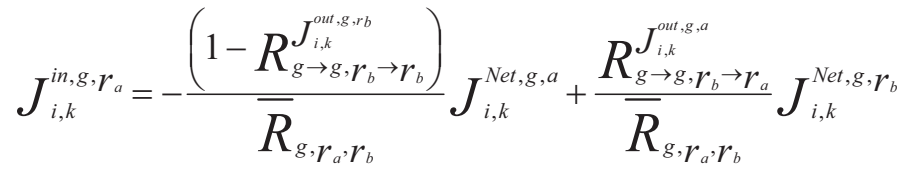

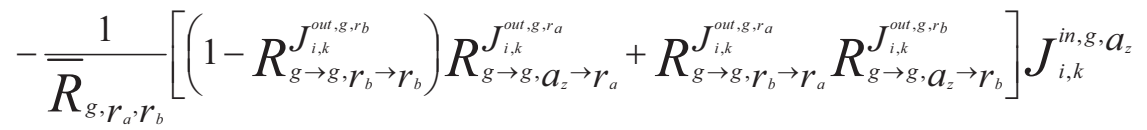

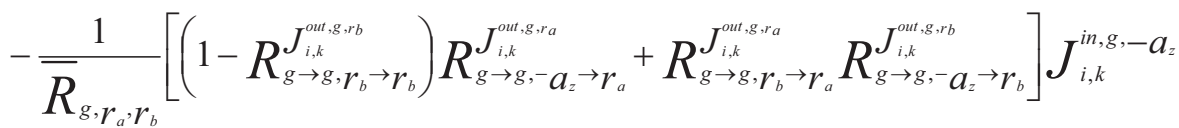

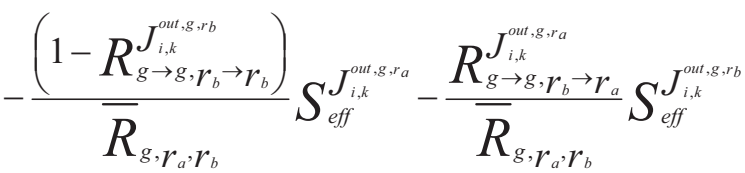

where $\bar{R}_{g, r_{a}, r_{b}}$ is expressed in Equation (26). The incoming axial currents are obtained in a similar manner as the radial incoming currents. Equations (23) and (24) are multiplied by similar coefficients and the resulting product equations are subtracted. The subtraction of the two product equations removes one of the incoming current and the resulting equation contains the incoming current of interest, the incoming radial currents, the axial net currents, and the effective sources of the exiting axial currents. The resulting expressions of the two incoming axial currents are

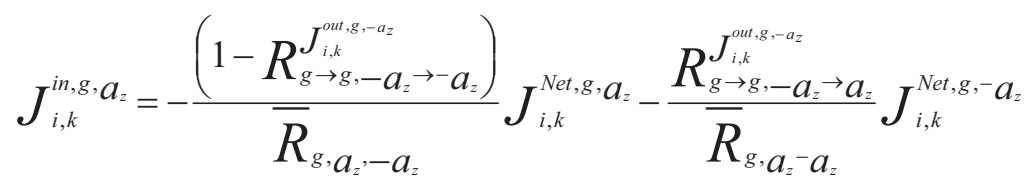

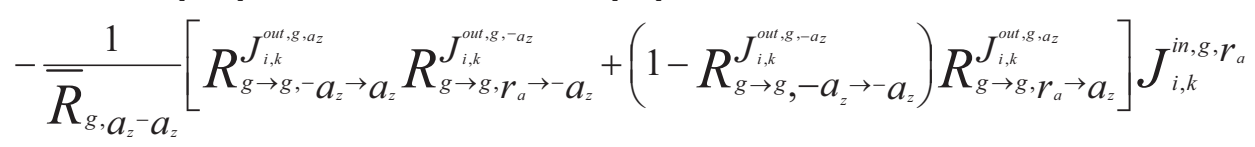

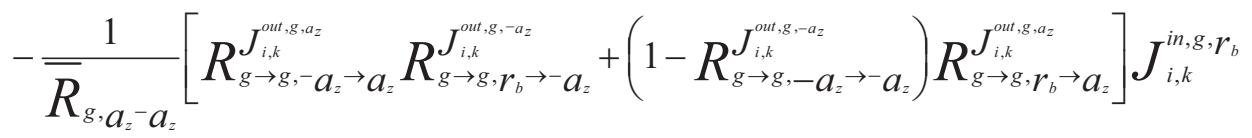

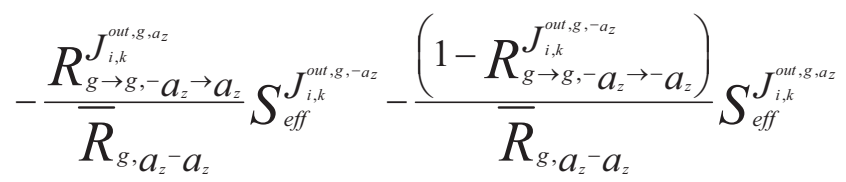

and 


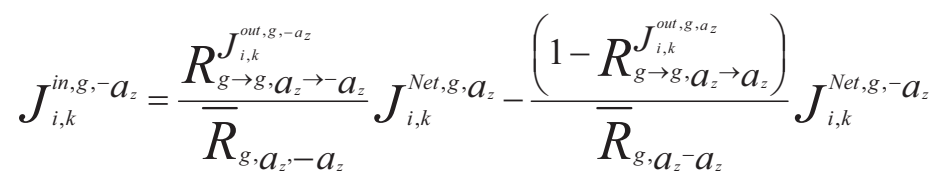

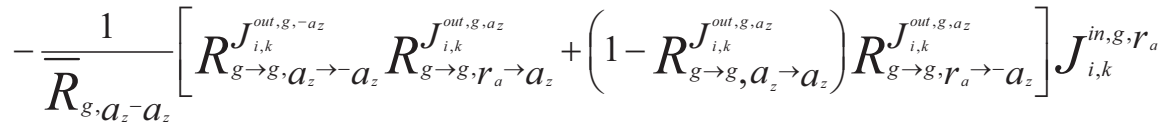

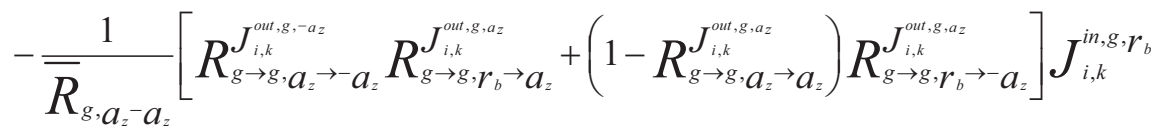

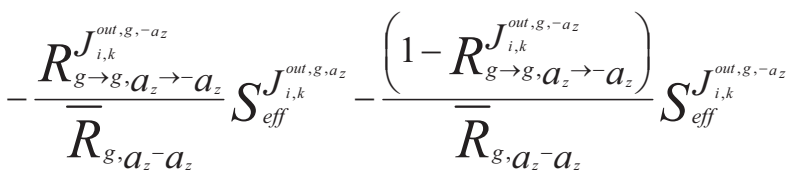

where

$$
\bar{R}_{g, a_{z}, a_{z}}=R_{g \rightarrow g,-a_{z} \rightarrow a_{z}}^{J_{i, k, g, a_{z}}^{\text {out }}} R_{g \rightarrow g, a_{z} \rightarrow-a_{z}}^{J_{i, k, g, a_{z}}^{\text {out }}}-\left(1-R_{g \rightarrow g, a_{z} \rightarrow a_{z}}^{J_{i, k}^{\text {out }, g, a_{z}}}\right)\left(1-R_{g \rightarrow g,-a_{z} \rightarrow-a_{z}}^{J_{i, k}^{\text {out }, g, a_{z}}}\right)
$$

To obtain the set of equations that contain only the incoming radial currents, the four edge net currents, and effective sources for the outgoing currents, the incoming axial currents of Equations (28) and (29) are substituted into Equations (26) and (27). After some algebra to collect terms for the radial incoming currents, net currents, and effective nodal sources for the outgoing current, the two resulting equations are

$$
\begin{aligned}
& -\frac{1}{\bar{R}_{g, r_{a}, r_{a}}} \bar{R}_{g, a_{z^{\prime},} a_{z}}\left[\bar{R}_{g, r_{a}, r_{a}} \bar{R}_{g, a_{z},-a_{z}}-R_{r_{a}}^{a_{z}} R_{a_{z}}^{r_{a}}-R_{r_{a}}^{-a_{z}} R_{-a_{z}}^{r_{a}}\right] J_{i, k}^{i n, g, r_{a}} \\
& +\frac{1}{\overline{R_{g, r_{a}, r_{a}}}}\left[R_{R_{, a_{z},-} a_{z}}^{a_{z}} R_{a_{z}}^{r_{a}}+R_{r_{a}}^{-a_{z}} R_{-a_{z}}^{r_{a}}\right] J_{i, k}^{i n, g, r_{b}} \\
& =-\frac{\left(1-R_{g \rightarrow g, r_{b} \rightarrow r_{b}}^{J_{i, t, g, r_{b}}}\right)}{\bar{R}_{g, r_{a}, r_{a}}} J_{i, k}^{N e t, g, r_{a}}+\frac{R_{g \rightarrow g, r_{b} \rightarrow r_{a}}^{J_{i, k, g, r_{a}}^{o u}}}{\bar{R}_{g, r_{a}, r_{b}}^{N e t, g, r_{b}}}
\end{aligned}
$$

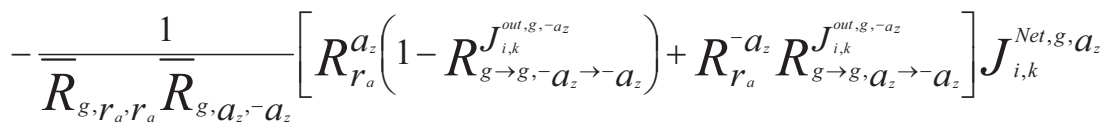

$$
\begin{aligned}
& +\frac{1}{\bar{R}_{g, \boldsymbol{r}_{a}, \boldsymbol{r}_{a}}} \bar{R}_{g, a_{z},-a_{z}}\left[R_{r_{a}}^{a_{z}} R_{g \rightarrow g,-a_{z} \rightarrow a_{z}}^{J_{i, k, g, a_{z}}^{\text {out }}}+R_{r_{a}}^{-a_{z}}\left(1-R_{g \rightarrow g, a_{z} \rightarrow a_{z}}^{J_{i, k, g, a_{z}}^{\text {out }}}\right)\right] J_{i, k}^{\text {Net, }, g,-a_{z}}
\end{aligned}
$$

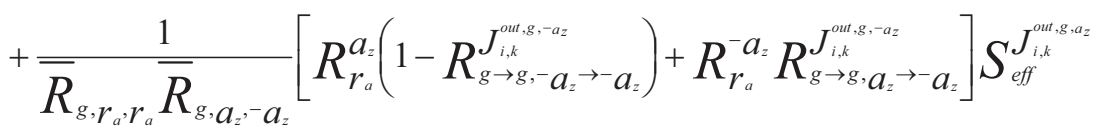

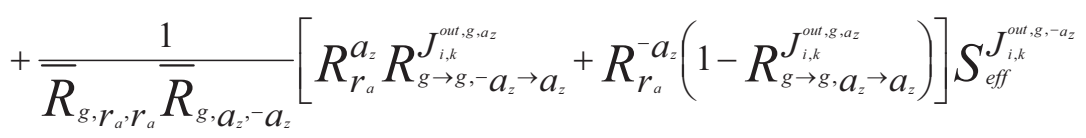

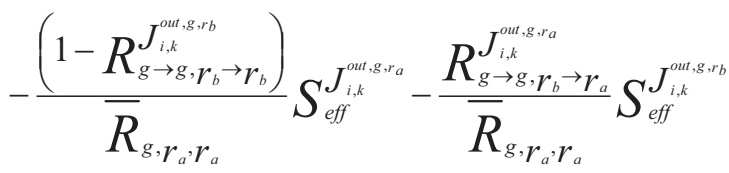


and

$$
\begin{aligned}
& -\frac{1}{R_{g, r_{a}, r_{a}}} \bar{R}_{g, a_{z},-a_{z}}\left[R_{r_{b}}^{a_{z}} R_{a_{z}}^{r_{b}}+R_{r_{b}}^{-a_{z}} R_{-a_{z}}^{r_{b}}\right] J_{i, k}^{i n, g, r_{a}} \\
& +\frac{1}{\bar{R}_{g, r_{a}, r_{a}} \bar{R}_{g, a_{z},-a_{z}}}\left[\bar{R}_{g_{, r_{a}, r_{a}}} \bar{R}_{g, a_{z},-a_{z}}-R_{r_{b}}^{a_{z}} R_{a_{z}}^{r_{b}}-R_{r_{b}}^{-a_{z}} R_{-a_{z}}^{r_{b}}\right] J_{i, k}^{i n, g, r_{b}} \\
& =-\frac{R_{g \rightarrow g, r_{a} \rightarrow r_{b}}^{J_{i, t, g, r_{b}}}}{R_{g, r_{a}, r_{a}}^{N e t, g, r_{a}}} J_{i, k}^{N}+\frac{\left(1-R_{g \rightarrow g, r_{a} \rightarrow r_{a}}^{J_{i, k, g, r_{a}}}\right.}{R_{g, r_{a}, r_{b}}} J_{i, k}^{N e t, g, r_{b}} \\
& -\frac{1}{\bar{R}_{g, r_{a}, r_{a}} \bar{R}_{g, a_{z},-} a_{z}}\left[R_{r_{b}}^{a_{z}}\left(1-R_{g \rightarrow g,-a_{z} \rightarrow-a_{z}}^{J_{i, k, g,-a_{z}}^{o u n}}\right)+R_{r_{b}}^{-a_{z}} R_{g \rightarrow g, a_{z} \rightarrow-a_{z}}^{J_{i, k, g,-a_{z}}^{o u n}}\right] J_{i, k}^{N e t, g, a_{z}}
\end{aligned}
$$

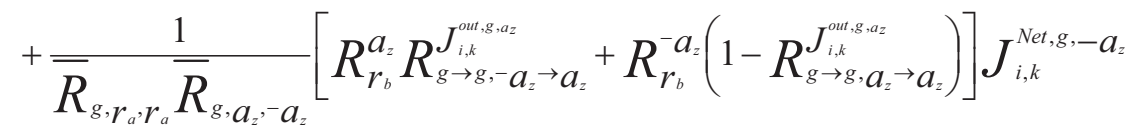

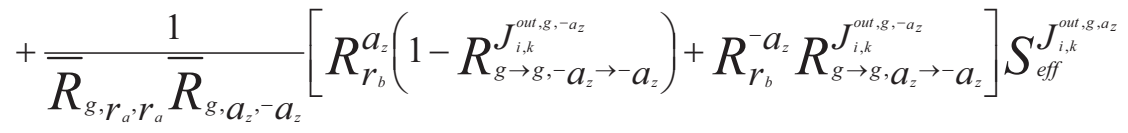

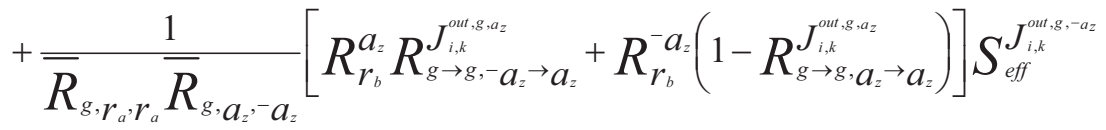

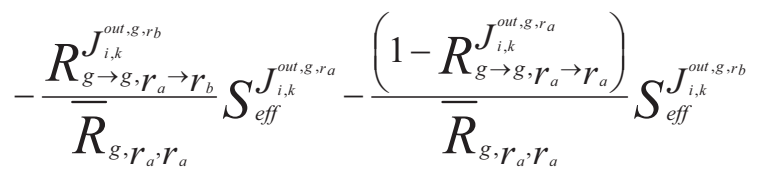

where

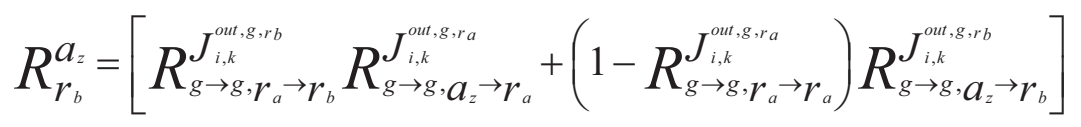

$$
\begin{aligned}
& R_{\boldsymbol{r}_{b}}^{-a_{z}}=\left[R_{g \rightarrow g, \boldsymbol{r}_{a} \rightarrow \boldsymbol{r}_{b}}^{J_{i, k}^{\text {out },, r_{b}}} R_{g \rightarrow g,-a_{z} \rightarrow \boldsymbol{r}_{a}}^{J_{i, k}^{\text {out }, g, r_{a}}}+\left(1-R_{g \rightarrow g, \boldsymbol{r}_{a} \rightarrow \boldsymbol{r}_{a}}^{J_{i, k}^{\text {out, }, r_{a}}}\right) R_{g \rightarrow g,-a_{z} \rightarrow \boldsymbol{r}_{b}}^{J_{i, k}^{\text {out }, r_{b}}}\right] \\
& R_{r_{a}}^{a_{z}}=\left[\left(1-R_{g \rightarrow g, r_{b} \rightarrow r_{b}}^{J_{i, k}^{\text {out }, g, r_{b}}}\right) R_{g \rightarrow g, a_{z} \rightarrow r_{a}}^{J_{i, k}^{\text {out, }, r_{a}}}+R_{g \rightarrow g, \boldsymbol{r}_{b} \rightarrow \boldsymbol{r}_{a}}^{J_{i, k}^{\text {out, }, r_{a}}} R_{g \rightarrow g, \boldsymbol{a}_{z} \rightarrow \boldsymbol{r}_{b}}^{J_{i, t, g, r_{b}}^{\text {out }}}\right] \\
& R_{r_{a}}^{-a_{z}}=\left[\left(1-R_{g \rightarrow g, r_{b} \rightarrow r_{b}}^{J_{i, k}^{\text {out, }, r_{b}}}\right) R_{g \rightarrow g,-a_{z} \rightarrow r_{a}}^{J_{i, k}^{\text {out }, g, r_{a}}}+R_{g \rightarrow g, r_{b} \rightarrow r_{a}}^{J_{i, k}^{\text {out }, g, r_{a}}} R_{g \rightarrow g,-a_{z} \rightarrow r_{b}}^{J_{i, t, g, r_{b}}^{\text {of }}}\right] \\
& R_{a_{z}}^{r_{a}}=\left[\left(1-R_{g \rightarrow g,-a_{z} \rightarrow-a_{z}}^{J_{i, k}^{\text {out },,-a_{z}}}\right) R_{g \rightarrow g, r_{a} \rightarrow a_{z}}^{J_{i, k}^{\text {out }, g, a_{z}}}+R_{g \rightarrow g,-a_{z} \rightarrow a_{z}}^{J_{i, k}^{\text {out }, a_{z}}} R_{g \rightarrow g, \boldsymbol{r}_{a} \rightarrow-a_{z}}^{J_{i, k}^{\text {out }, g,-a_{z}}}\right] \\
& R_{a_{z}}^{r_{b}}=\left[\left(1-R_{g \rightarrow g,-a_{z} \rightarrow-a_{z}}^{J_{i, k}^{\text {out },,-a_{z}}}\right) R_{g \rightarrow g, \boldsymbol{r}_{b} \rightarrow a_{z}}^{J_{i, k}^{\text {out }, g, a_{z}}}+R_{g \rightarrow g,-a_{z} \rightarrow a_{z}}^{J_{i, k}^{\text {out }, a_{z}, a_{z}}} R_{g \rightarrow g, \boldsymbol{r}_{b} \rightarrow-a_{z}}^{J_{i, k}^{\text {out }, g,-a_{z}}}\right]
\end{aligned}
$$




$$
R_{-a_{z}}^{r_{a}}=\left[\left(1-R_{g \rightarrow g, a_{z} \rightarrow a_{z}}^{J_{i, k}^{\text {out }, g, a_{z}}}\right) R_{g \rightarrow g, \boldsymbol{r}_{a} \rightarrow-a_{z}}^{J_{i, k}^{\text {out }, g, a_{z}}}+R_{g \rightarrow g, a_{z} \rightarrow-a_{z}}^{J_{i, k}^{\text {out }, g,-a_{z}}} R_{g \rightarrow g, \boldsymbol{r}_{a} \rightarrow a_{z}}^{J_{i, k}^{\text {out }, g, a_{z}}}\right]
$$

and

$$
R_{-a_{z}}^{r_{b}}=\left[\left(1-R_{g \rightarrow g, a_{z} \rightarrow a_{z}}^{J_{i, k}^{\text {out, }, a_{z}}}\right) R_{g \rightarrow g, r_{b} \rightarrow-a_{z}}^{J_{i, k}^{\text {out, },, a_{z}}}+R_{g \rightarrow g, a_{z} \rightarrow-a_{z}}^{J_{i, k}^{\text {out }, g, a_{z}}} R_{g \rightarrow g, r_{b} \rightarrow a_{z}}^{J_{i, k}^{\text {out, }, a_{z}}}\right]
$$

The matrix equations formed by Equations (31) and (32)Error! Reference source not found. are analytically inverted to yield expressions for the inward radial currents as

$$
\begin{aligned}
& J_{i, k}^{i n, g, r_{a}}=C_{i, k, g, r_{a}}^{r_{a}} J_{i, k}^{N e t, g, r_{a}}+C_{i, k, g, r_{a}}^{r_{b}} J_{i, k}^{N e t, g, r_{a}}+C_{i, k, g, r_{a}}^{a_{z}} J_{i, k}^{\text {Net,g, } a_{z}}+C_{i, k, g, r_{a}}^{-a_{z}} J_{i, k}^{N e t, g,-a_{z}}
\end{aligned}
$$

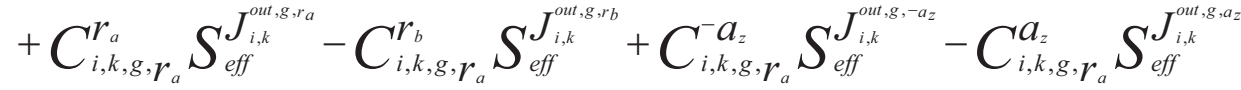

and

$$
\begin{aligned}
& J_{i, k}^{i n, g, r_{b}}=C_{i, k, g, r_{b}}^{r_{a}} J_{i, k}^{N e t, g, r_{a}}+C_{i, k, g, r_{b}}^{r_{b}} J_{i, k}^{N e t, g, r_{a}}+C_{i, k, g, r_{b}}^{a_{z}} J_{i, k}^{N e t, g, a_{z}}+C_{i, k, g, r_{b}}^{-} J_{i, k}^{N e t, g,-a_{z}}
\end{aligned}
$$

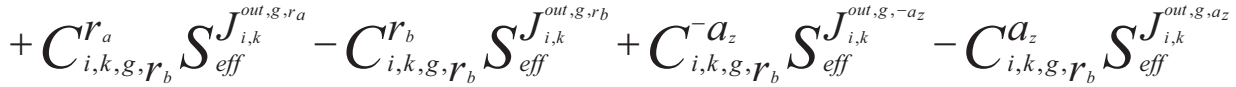

The coefficients that are in front of the net currents and source terms are composed of response functions. The coefficients are:

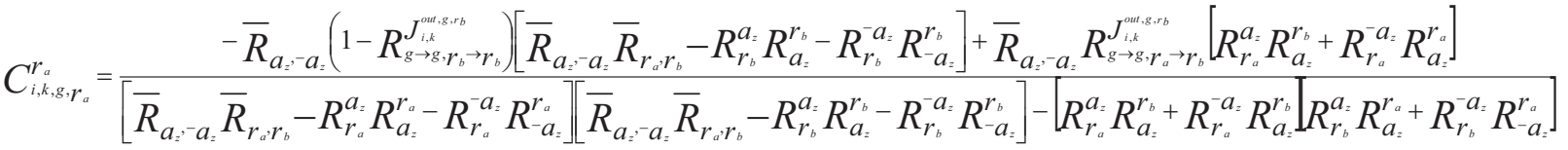

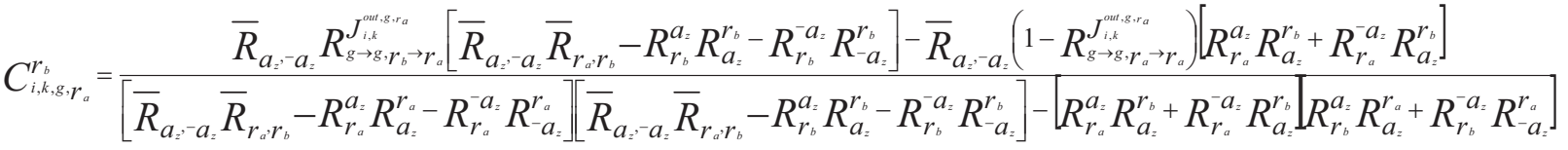

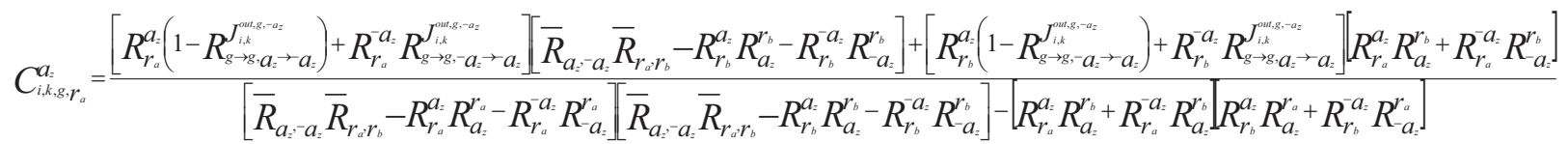

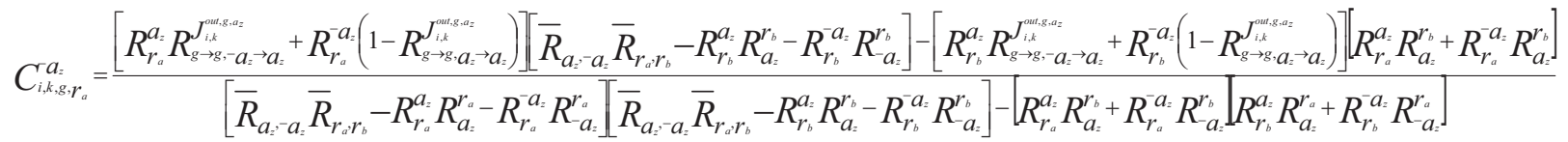

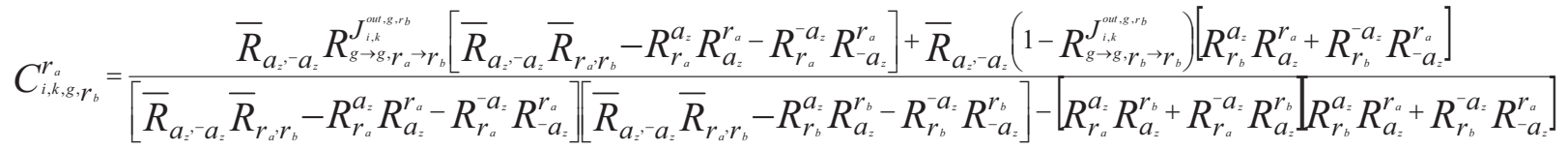

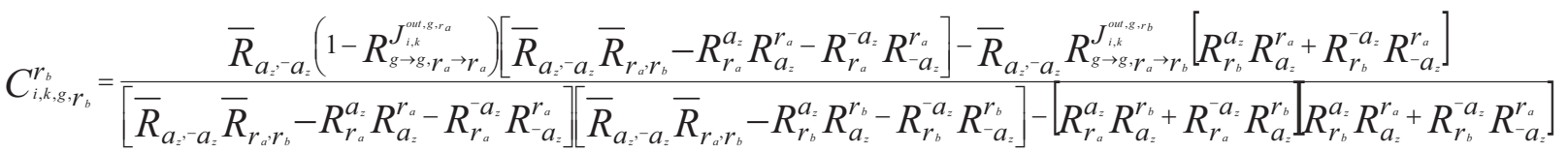




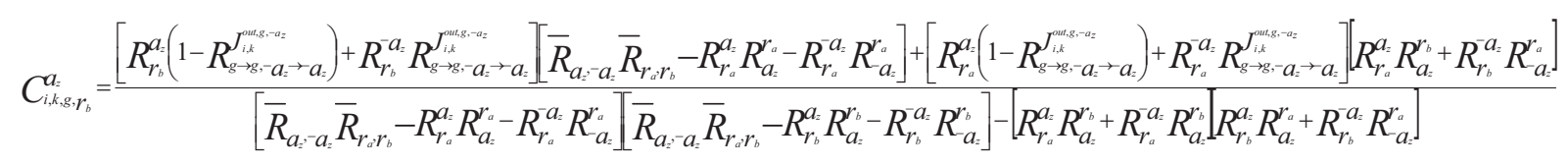

and

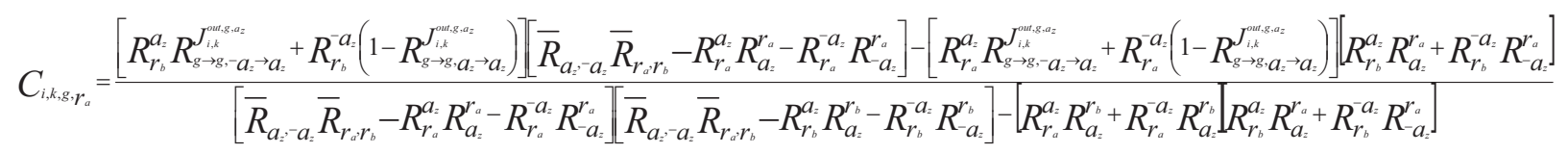

To obtain the set of equations that contain only the incoming axial currents, the four edge net currents, and effective sources for the outgoing currents, the incoming radial currents of Equations (26) and (27) are substituted into Equations (28) and (29). After some algebra to collect terms for the incoming axial currents, net currents, and effective nodal sources for the outgoing current, the two resulting equations are

$$
\begin{aligned}
& \overline{\bar{R}_{g, a_{z}, a_{z}}} \frac{1}{R_{g, r_{a}, r_{b}}}\left[\bar{R}_{g, a_{z},-a_{z}} \bar{R}_{g, r_{a}, r_{b}}-R_{r_{a}}^{a_{z}} R_{a_{z}}^{r_{a}}-R_{r_{b}}^{a_{z}} R_{a_{z}}^{r_{b}}\right] J_{i, k}^{i n, g, a_{z}} \\
& -\frac{1}{\overline{R_{g, a_{z},-a_{z}}}} \overline{R_{g, r_{a}, r_{b}}}\left[R_{a_{z}}^{r_{a}} R_{r_{a}}^{-a_{z}}+R_{a_{z}}^{r_{b}} R_{r_{b}}^{-a_{z}}\right] J_{i, k}^{i n, g,-a_{z}} \\
& =\frac{\left(1-R_{g \rightarrow g,-a_{z} \rightarrow-}^{J_{i, k}^{o u, g,-a_{z}}}\right)}{\bar{R}_{g, a_{z},-a_{z}}} J_{i, k}^{N e t, g, a_{z}}-\frac{R_{g \rightarrow g,-a_{z} \rightarrow a_{z}}^{J_{i, k, g,-a_{z}}^{o f}} J_{i, k}^{N e t, g,-a_{z}}}{\bar{R}_{g, a_{z}-a_{z}}}
\end{aligned}
$$

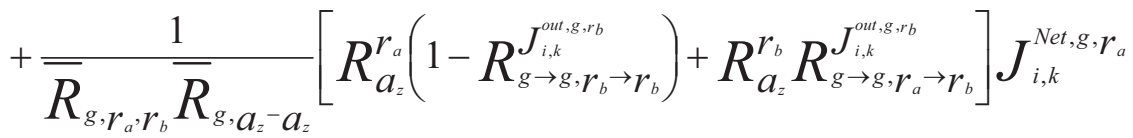

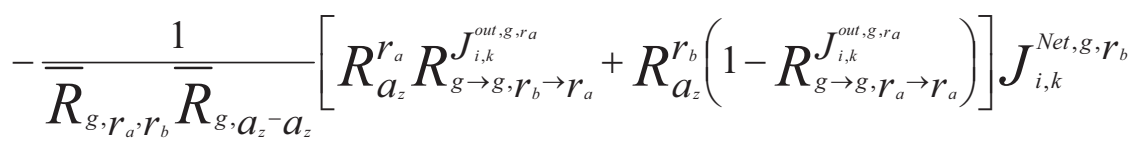

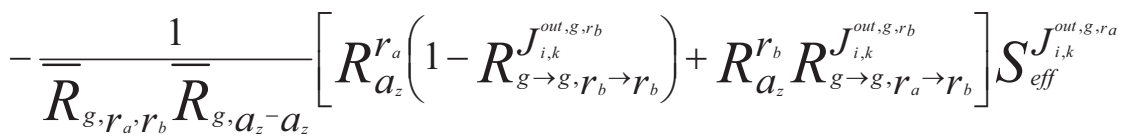

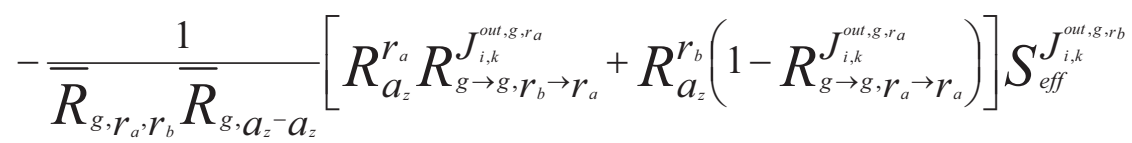

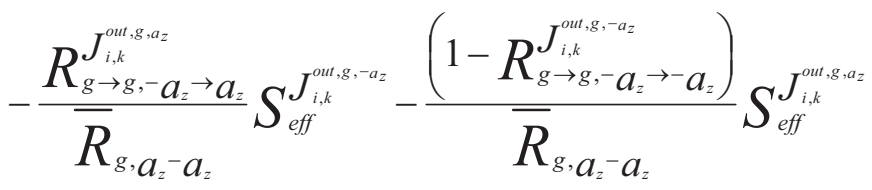

and 


$$
\begin{aligned}
& \frac{1}{\bar{R}_{g, a_{z},-a_{z}} \bar{R}_{g, r_{a}, r_{b}}}\left[\bar{R}_{g, a_{z},-a_{z}} \bar{R}_{g, r_{a}, r_{b}}-R_{r_{a}}^{-a_{z}} R_{-a_{z}}^{r_{a}}-R_{r_{b}}^{-a_{z}} R_{-a_{z}}^{r_{b}}\right] J_{i, k}^{i n, g,-a_{z}} \\
& +\frac{1}{\bar{R}_{g, a_{z}, a_{z}} \bar{R}_{g, r_{a}, r_{b}}}\left[R_{-a_{z}}^{r_{a}} R_{r_{a}}^{-a_{z}}+R_{-a_{z}}^{r_{b}} R_{r_{b}}^{-a_{z}}\right] J_{i, k}^{i n, g, a_{z}}
\end{aligned}
$$

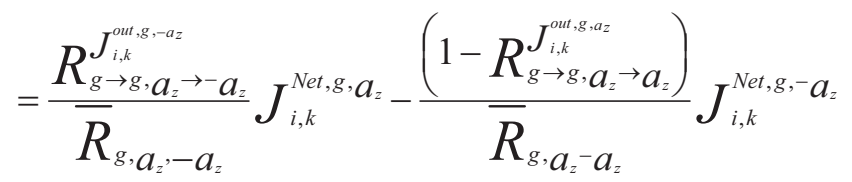

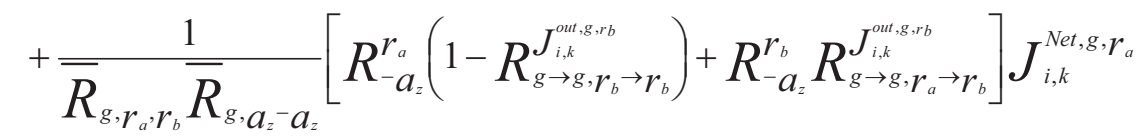

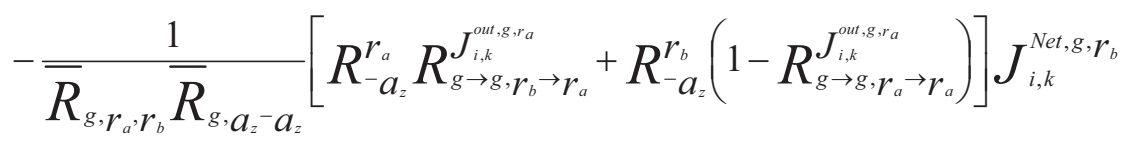

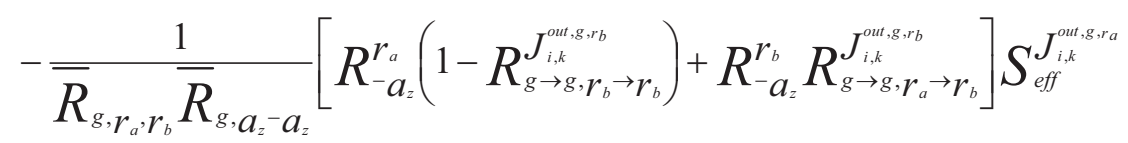

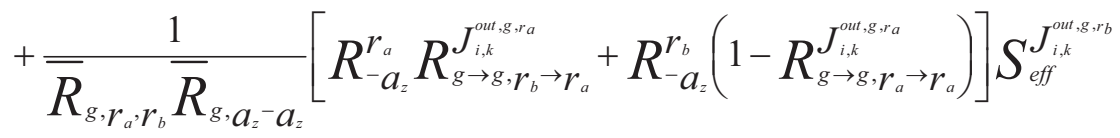

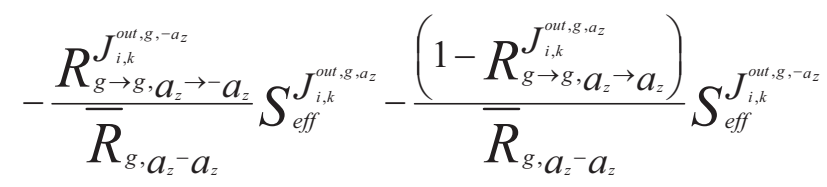

Equations (43) and (44) are solved algebraically for the axial incoming currents to obtain

$$
\begin{aligned}
& J_{i, k}^{i n, g, a_{z}}=C_{i, k, g, a_{z}}^{r_{a}} J_{i, k}^{\mathrm{Net,g,}, \boldsymbol{r}_{a}}+C_{i, k, g, a_{z}}^{r_{b}} J_{i, k}^{\mathrm{Net,g,} \cdot r_{a}}+C_{i, k, g, a_{z}}^{a_{z}} J_{i, k}^{\mathrm{Net,g}, a_{z}}+C_{i, k, g, a_{z}}^{-a_{z}} J_{i, k}^{\mathrm{Net,g,}, a_{z}}
\end{aligned}
$$

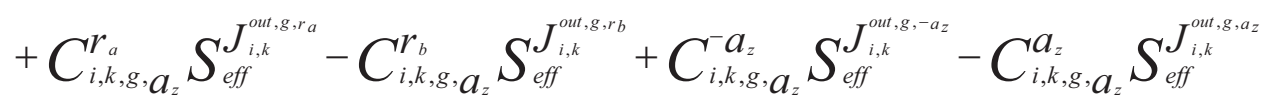

and

$$
\begin{aligned}
& J_{i, k}^{i n, g,-a_{z}}=C_{i, k, g,-a_{z}}^{r_{a}} J_{i, k}^{N e t, g, r_{a}}+C_{i, k, g,-a_{z}}^{r_{b}} J_{i, k}^{N e t, g, r_{a}}+C_{i, k, g,-a_{z}}^{a_{z}} J_{i, k}^{\text {Net, g, } a_{z}}+C_{i, k, g,-a_{z}}^{-a_{z}} J_{i, k}^{\mathrm{Net,g,-} a_{z}}
\end{aligned}
$$

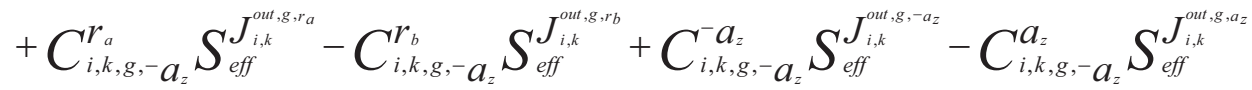

The coefficients are given as

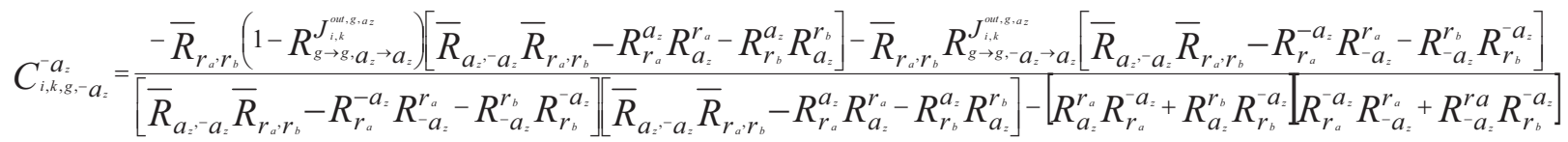

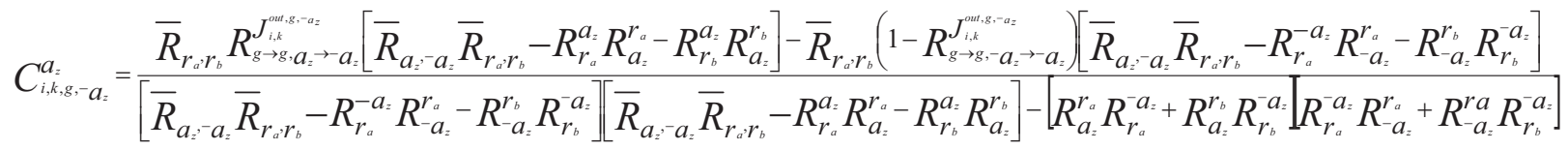




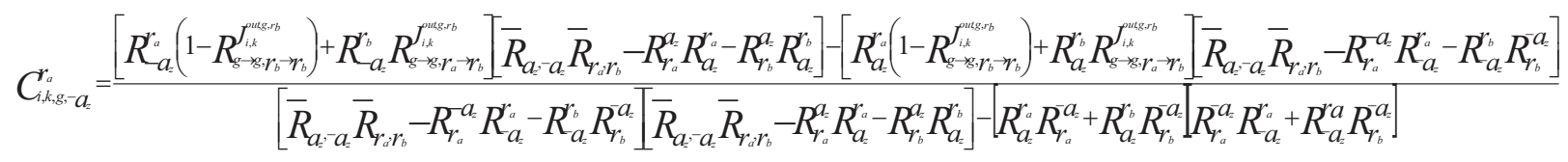

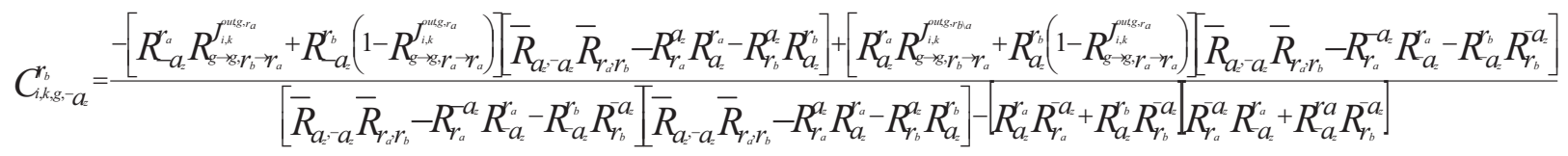

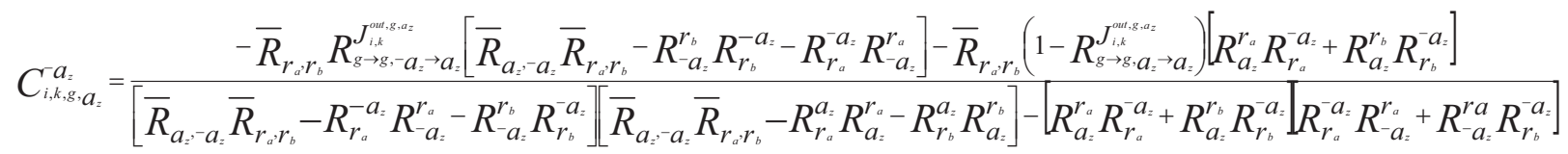

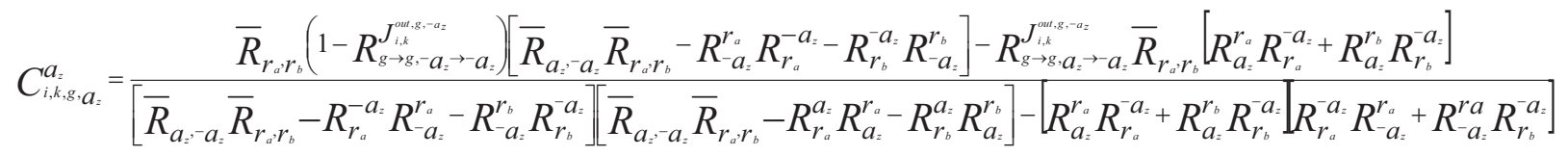

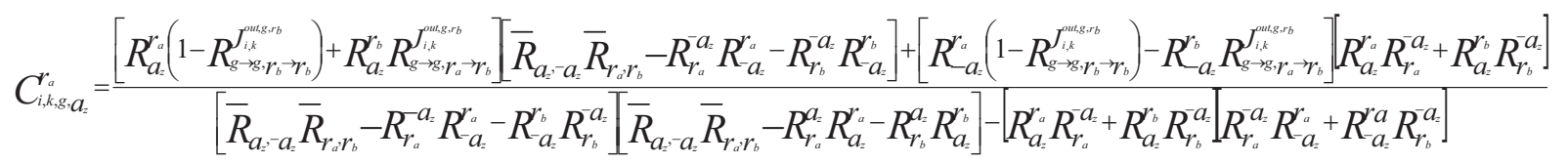

and

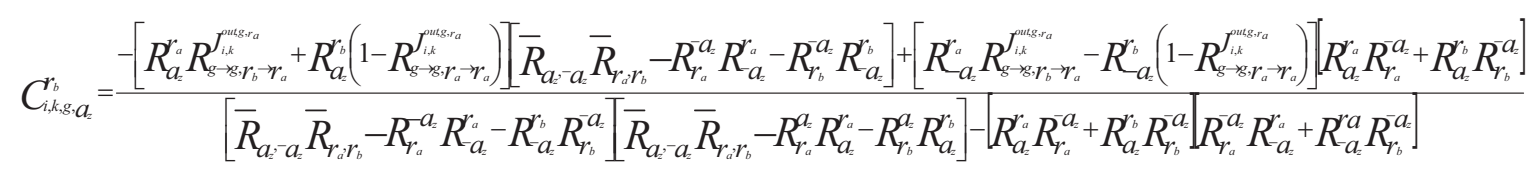

Equations (41), (42), (43), (45), and (46) express the incoming node currents in terms of the net node boundary currents. To obtain the averaged balance equation, these incoming currents equations are substituted into the response equation for the response node average scalar flux. The equation for the average flux of the response node is

$$
\Phi^{g}=\sum_{g^{\prime}=1}^{G} \sum_{f=r_{a} \cdot r_{b^{\prime}} a_{z^{\prime}}-a_{z}} R_{g^{\prime} \rightarrow g, f}^{\Phi^{g}} J_{i, k}^{i n, g^{\prime}, f}
$$

Like the expressions for the outgoing current, the effective sources for the node are grouped together in Equation (47). The equations for the average flux then becomes

$$
\Phi^{g}=R_{g \rightarrow g, r_{a}}^{\Phi^{g}} J_{i, k}^{i n, g, r_{a}}+R_{g \rightarrow g, r_{b}}^{\Phi^{g}} J_{i, k}^{i n, g, r_{b}}+R_{g \rightarrow g, a_{z}}^{\Phi^{g}} J_{i, k}^{i n, g, a_{z}}+R_{g \rightarrow g,-a_{z}}^{\Phi^{g}} J_{i, k}^{i n, g,-a_{z}}+\widetilde{S}_{e f f}^{\Phi_{g}}
$$

where

$$
S_{e f f^{\prime}}^{\Phi_{g}}=\sum_{g^{\prime}=1, g^{\prime} \neq g}^{G} \sum_{f=r_{a^{\prime}} r_{b^{\prime}} a_{z}-a_{z}} R_{g^{\prime} \rightarrow g, f}^{\Phi_{g}} J_{i, k}^{i n, g^{\prime}, f}
$$


The source given by Equation (49) is held constant for node (i,k) and energy group g. The incoming currents are expressed in Equations (41), (42), (43), (45), and (46), and are substituted into Equation (48). After some algebra to collect terms for the net boundary currents and the effective nodal sources, the resulting equation is

$$
\begin{aligned}
& H_{i, k}^{g, r_{a}} J_{i, k}^{N e t, g, r_{a}}+H_{i, k}^{g, r_{b}} J_{i, k}^{N e t, g, r_{b}}+H_{i, k}^{g, a_{z}} J_{i, k}^{N e t, g, a_{z}}+H_{i, k}^{g,-a_{z}} J_{i, k}^{N e t, g,-a_{z}}+\Phi^{g}
\end{aligned}
$$

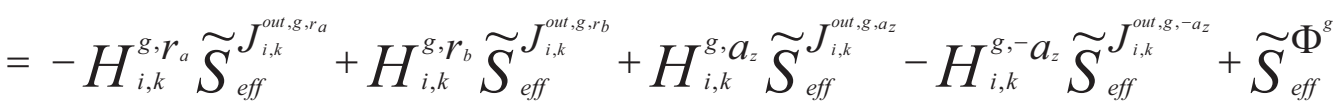

where

$$
\begin{aligned}
& H_{i, k}^{g, r_{a}}=-R_{g \rightarrow g, r_{b}}^{\Phi_{g}} C_{g, r_{b}}^{r_{a}}-R_{g \rightarrow g, r_{a}}^{\Phi_{g}} C_{g, r_{a}}^{r_{a}}-R_{g \rightarrow g, a_{z}}^{\Phi_{g}} C_{g, a_{z}}^{r_{a}}-R_{g \rightarrow g,-a_{z}}^{\Phi_{g}} C_{g,-a_{z}}^{r_{a}} \\
& H_{i, k}^{g, r_{b}}=-R_{g \rightarrow g, r_{b}}^{\Phi_{g}} C_{g, r_{b}}^{r_{b}}-R_{g \rightarrow g, r_{a}}^{\Phi_{g}} C_{g, r_{a}}^{r_{b}}-R_{g \rightarrow g, a_{z}}^{\Phi_{g}} C_{g, a_{z}}^{r_{b}}-R_{g \rightarrow g,-a_{z}}^{\Phi_{g}} C_{g,-a_{z}}^{r_{b}} \\
& H_{i, k}^{g, a_{z}}=-R_{g \rightarrow g, r_{b}}^{\Phi_{g}} C_{g, r_{b}}^{a_{z}}-R_{g \rightarrow g, r_{a}}^{\Phi_{g}} C_{g, r_{a}}^{a_{z}}-R_{g \rightarrow g, a_{z}}^{\Phi_{g}} C_{g, a_{z}}^{a_{z}}-R_{g \rightarrow g,-a_{z}}^{\Phi_{g}} C_{g,-a_{z}}^{a_{z}}
\end{aligned}
$$

and

$$
H_{i, k}^{g,-a_{z}}=-R_{g \rightarrow g, r_{b}}^{\Phi_{g}} C_{g, r_{b}}^{-} a_{z}-R_{g \rightarrow g, r_{a}}^{\Phi_{g}} C_{g, r_{a}}^{-a_{z}}-R_{g \rightarrow g, a_{z}}^{\Phi_{g}} C_{g, a_{z}}^{-} a_{z}-R_{g \rightarrow g,-a_{z}}^{\Phi_{g}} C_{g,-a_{z}}^{-} a_{z}
$$

Equation (50) is the spatially averaged balance equation for a response node. 


\section{DERIVATION OF THE COMMON AXIAL INTERFACE NET CURRENT EQUATIONS}

The equations for the axial net currents are derived in this section. The equation of the axial net currents are important because they are substituted into an appropriate balance equation to obtain either the three- or five-striped CMFD (nodal) form of the equation for the average scalar flux. They are also important for obtaining the net current on the axial boundary after values for the scalar fluxes are obtained. Derivations are given in this section for the net axial current equations that include three cases: the case when two response nodes are axially adjacent to each other, the case when a response node is next to a diffusion treated node, and the case when the response node is at the reactor boundary.

\subsection{Common Interface Current for Two Response Nodes Adjoining an Axial Boundary}

The equation for the common interface current for two adjacent response nodes is derived in this section. The derivation starts by setting equal the common boundary fluxes from the two adjacent nodes, and then expanding the boundary fluxes in terms of response functions multiplied by the respective incoming boundary currents. All the incoming currents are replaced by equations relating the incoming currents for a node to the net currents on the node boundary. These equations were derived in the section for the balance equation of a response node. The terms for the net currents are collected, and the net currents for the opposite faces of the adjoined boundary are replaced by an expression derived from the balance equation for each response node. The terms with the net current on the adjoining boundary are algebraically collected and the net current on the adjoining boundary is algebraically obtained.

The derivation starts by obtaining the side (surface) scalar flux as a response to the incoming currents, as obtained from either a numerical calculation or from an analytical derivation. The axial response function equations for the scalar flux for the node top and node bottom are

$\phi_{i, k}^{g, a_{z}}=\sum_{g^{\prime}=1}^{G} \sum_{f=\boldsymbol{r}_{a^{\prime}}, \boldsymbol{r}_{b^{\prime}}, \boldsymbol{a}_{z^{\prime}},-} R_{a_{z}}^{\phi_{g^{\prime} \rightarrow g, f \rightarrow a_{z}}^{g, a_{z}}} J_{i, k}^{i n, g^{\prime}, f}$

and

$\phi_{i, k}^{g,-a_{z}}=\sum_{g^{\prime}=1}^{G} \sum_{f=r_{a}, r_{b}, a_{z^{\prime}},-} R_{z} R_{g^{\prime} \rightarrow g, f \rightarrow-}^{\phi_{i, ~}^{g,-a_{z}}} J_{i, k}^{i n, g^{\prime}, f}$

In Equations (51) and (52), the edges of the node are denoted by $r_{a}, r_{b}, a_{z}$ and $-a_{z}$. The energy group index is given by $g$ and the maximum number of energy groups is denoted by $G$. The index $i$ denotes the radial spatial node index and the index $k$ denotes the axial spatial node index. The incoming currents for the energy groups that are not the current energy group $g$ are held constant for the solution of fluxes and currents in group $g$. These terms are effective source terms for the response nodes. Thus Equations (51) and (52) are rewritten as

$\phi_{i, k}^{g, a_{z}}=R_{g \rightarrow g, r_{a} \rightarrow a_{z}}^{\phi_{i, a_{z}}^{g}} J_{i, k}^{i, g, \boldsymbol{r}_{a}}+R_{g \rightarrow g, \boldsymbol{r}_{b} \rightarrow a_{z}}^{\phi_{i, k}^{g, a_{z}}} J_{i, k}^{i n, g, r_{b}}+R_{g \rightarrow g, a_{z} \rightarrow a_{z}}^{\phi_{i, k}^{g, a_{z}}} J_{i, k}^{i n, g, a_{z}}+R_{g \rightarrow g,-a_{z} \rightarrow a_{z}}^{\phi_{i, k}^{g, a_{z}}} J_{i, k}^{i n, g,-a_{z}}+S_{e f f}^{\phi_{i, k}^{g, a, a_{z}}}$

and

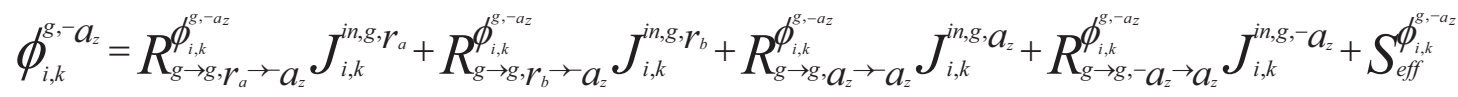


where

$$
\widetilde{S}_{\text {eff }}^{\phi_{i, k}^{g, a_{z}}}=\sum_{g^{\prime}=1, g^{\prime} \neq g}^{G} \sum_{f=r_{a^{\prime}}, r_{b^{\prime}} a_{z^{\prime}},-a_{z}} R_{g^{\prime} \rightarrow g, f \rightarrow a_{z}}^{\phi_{i, k}^{g, a_{z}}} J_{i, k}^{i n, g^{\prime}, f}
$$

and

$$
\widetilde{S}_{e f f}^{\phi_{i, k}^{g,-a_{z}}}=\sum_{g^{\prime}=1, g^{\prime} \neq g}^{G} \sum_{f=r_{a^{\prime}} \cdot r_{b^{\prime}} a_{z^{\prime}},-} a_{z} R_{g^{\prime} \rightarrow g, f \rightarrow-}^{\phi_{i, k}^{g,-a_{z}}} J_{i, k}^{i n, g^{\prime}, f}
$$

Equations (55) and (56) are the effective source terms for the scalar flux response equations. Next, for the interface between the response node $(\mathrm{i}, \mathrm{k})$ and $(\mathrm{i}, \mathrm{k}+1)$, the top axial scalar flux of node $(\mathrm{i}, \mathrm{k})$ is set equal to the bottom axial scalar flux equation of node $(\mathrm{i}, \mathrm{k}+1)$. The equation that results is

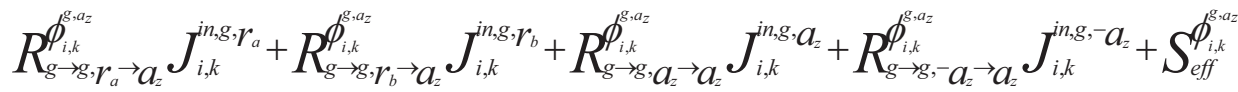

$$
\begin{aligned}
& =R_{g \rightarrow g, r_{a} \rightarrow a_{z}}^{\phi_{i, k+1}^{\mathrm{g}, a_{z}}} J_{i, k+1}^{i n, g, r_{a}}+R_{g \rightarrow g, r_{b} \rightarrow a_{z}}^{\phi_{i, k+1}^{\mathrm{g}, a_{z}}} J_{i, k+1}^{i n, g, r_{b}}+R_{g \rightarrow g, a_{z} \rightarrow a_{z}}^{\phi_{i, k+1}^{\mathrm{g},-a_{z}}} J_{i, k+1}^{i n, g, a_{z}}+R_{g \rightarrow g,-a_{z} \rightarrow a_{z}}^{\phi_{i, k+1}^{\mathrm{g},-a_{z}}} J_{i, k+1}^{i,, g,-a_{z}}+S_{e f f}^{\phi_{i, k+1}^{\mathrm{g},-a_{z}}} .
\end{aligned}
$$

Equations (41), (42), (45), and (46), which express the incoming currents in terms of the net interface currents, are substituted into Equation (57). Algebra manipulations are performed to collect net interface current terms. The resulting equation is

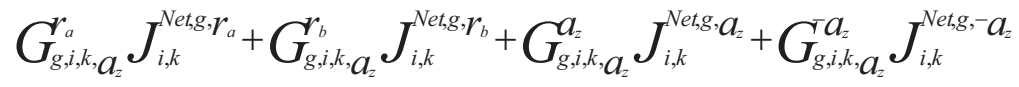

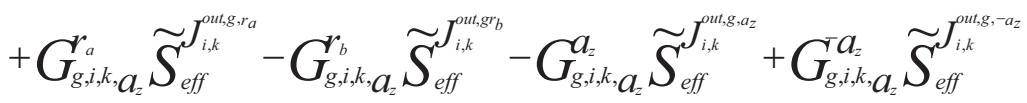

$$
\begin{aligned}
& =G_{g, i, k+1,-a_{z}}^{r_{a}} J_{i, k+1}^{\text {Net, }, r_{a}}+G_{g, i, k+1,-}^{r_{z}} J_{i, k+1}^{\text {Netg, } \boldsymbol{r}_{b}}+G_{g^{\prime}, i, k+1,-}^{a_{z}} J_{i, k+1}^{\text {Net, }, a_{z}}+G_{g^{\prime}, i, k+1,-}^{a_{z}} J_{i, k+1}^{\text {Netg, }, a_{z}}
\end{aligned}
$$

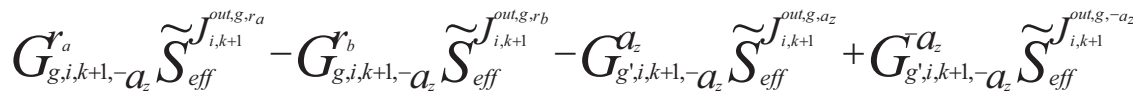

$$
\begin{aligned}
& +S_{e f f}^{\phi_{i, k+1}^{g,-a_{z}}}-S_{e f f}^{\phi_{i, k}^{g, a z}}
\end{aligned}
$$

The coefficients for the net currents are

$$
\begin{aligned}
& G_{g, i, k, a_{z}}^{a_{a}}=R_{g \rightarrow,, r_{a} \rightarrow a_{z}}^{\phi_{i, k}^{a_{z}}} C_{i, k, g, r_{a}}^{r_{a}}+R_{g \rightarrow g, r_{b} \rightarrow a_{z}}^{\phi_{i, k}^{a_{z}}} C_{i, k, g, r_{b}}^{r_{a}}+R_{g \rightarrow g, a_{z} \rightarrow a_{z}}^{\phi_{i, k}^{a_{z}}} C_{i, k, g, a_{z}}^{r_{a}}+R_{g \rightarrow, g,-a_{z} \rightarrow a_{z}}^{\phi_{i, k}^{a_{z}}} C_{i, k, g,-a_{z}}^{r_{a}} \\
& G_{g, i, k, a_{z}}^{r_{b}}=R_{g \rightarrow g, r_{a} \rightarrow a_{z}}^{\phi_{i, k}^{a_{z}}} C_{i, k, g, r_{a}}^{r_{b}}+R_{g \rightarrow g, r_{b} \rightarrow a_{z}}^{\phi_{i, k}^{a_{z}}} C_{i, k, g, r_{b}}^{r_{b}}+R_{g \rightarrow g, a_{z} \rightarrow a_{z}}^{\phi_{i, k}^{a_{z}}} C_{i, k, g, a_{z}}^{r_{b}}+R_{g \rightarrow g,-a_{z} \rightarrow a_{z}}^{\phi_{i, k}^{a_{z}}} C_{i, k, g,-}^{r_{b}} \\
& G_{g, i, k, a_{z}}^{a_{z}}=R_{g \rightarrow g, r_{a} \rightarrow a_{z}}^{\phi_{i, k}^{a_{z}}} C_{i, k, g, r_{a}}^{a_{z}}+R_{g \rightarrow g, r_{b} \rightarrow a_{z}}^{\phi_{i, k}^{a_{z}}} C_{i, k, g, r_{b}}^{a_{z}}+R_{g \rightarrow g, a_{z} \rightarrow a_{z}}^{\phi_{i, k}^{a_{z}}} C_{i, k, g, a_{z}}^{a_{z}}+R_{g \rightarrow g,-a_{z} \rightarrow a_{z}}^{\phi_{i, k}^{a_{z}}} C_{i, k, g,-a_{z}}^{a_{z}} \\
& G_{g, i, k, a_{z}}^{a_{z}}=R_{g \rightarrow g, r_{a} \rightarrow a_{z}}^{\phi_{i, k}^{a_{z}}} C_{i, k, g, r_{a}}^{a_{z}}+R_{g \rightarrow g, \boldsymbol{r}_{b} \rightarrow a_{z}}^{\phi_{i, k}^{a_{z}}} C_{i, k, g, r_{b}}^{a_{z}}+R_{g \rightarrow g, a_{z} \rightarrow a_{z}}^{\phi_{i, k}^{a_{z}}} C_{i, k, g, a_{z}}^{a_{z}}+R_{g \rightarrow g,-a_{z} \rightarrow a_{z}}^{\phi_{i, k}^{a_{z}}} C_{i, k, g,-a_{z}}^{a_{z}}
\end{aligned}
$$




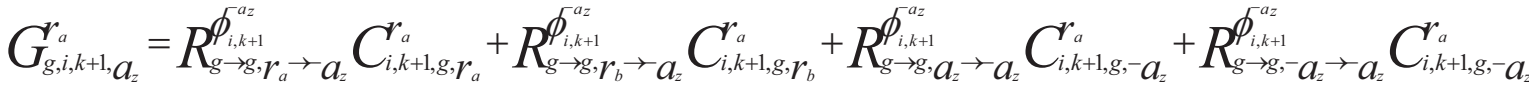

$$
\begin{aligned}
& G_{g, i, k+1, a_{z}}^{r_{b}}=R_{g \rightarrow g, r_{a} \rightarrow a_{z}}^{\oint_{i, k+1}^{a_{z}}} C_{i, k+1, g, r_{a}}^{r_{b}}+R_{g \rightarrow g, r_{b} \rightarrow a_{z}}^{\oint_{i, k+1}^{a_{z}}} C_{i, k+1, g, r_{b}}^{r_{b}}+R_{g \rightarrow g, a_{z} \rightarrow a_{z}}^{\oint_{i, k+1}^{a_{z}}} C_{i, k+1, g,-a_{z}}^{r_{b}}+R_{g \rightarrow g,-a_{z} \rightarrow a_{z}}^{\phi_{i, k+1, k+1, g,-}^{a_{z}}} C_{z}^{r_{b}} \\
& G_{g, i, k+1, a_{2}}^{a_{z}}=R_{g \rightarrow g, r_{a} \rightarrow a_{z}}^{\oint_{i, k+1}^{a_{z}}} C_{i, k+1, g, r_{a}}^{a_{z}}+R_{g \rightarrow g, r_{b} \rightarrow a_{z}}^{\oint_{i, k+1}^{a_{z}}} C_{i, k+1, g, r_{b}}^{a_{z}}+R_{g \rightarrow g, a_{z} \rightarrow a_{z}}^{\oint_{i, k+1}^{a_{z}}} C_{i, k+1, g,-a_{z}}^{a_{z}}+R_{g \rightarrow g,-a_{z} \rightarrow a_{z}}^{\oint_{i, k}^{a_{z}}} C_{i, k+1, g,-a}^{a_{z}}
\end{aligned}
$$

and

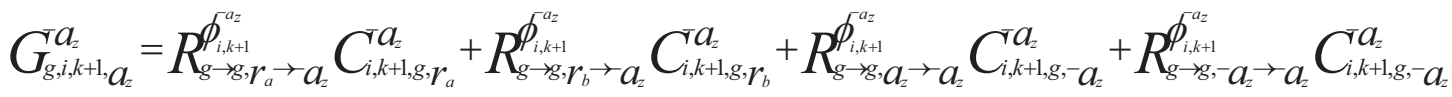

The net currents on the opposite boundary on each side of the common interface boundary are replaced with expressions obtained from the response balance formulation. The substitution into Equation (58) yields

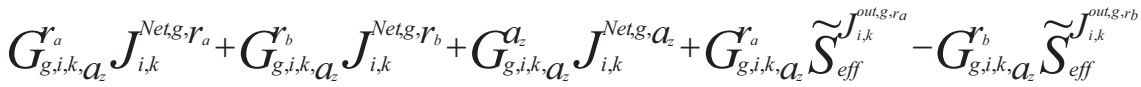

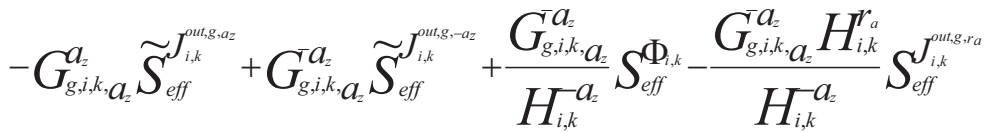

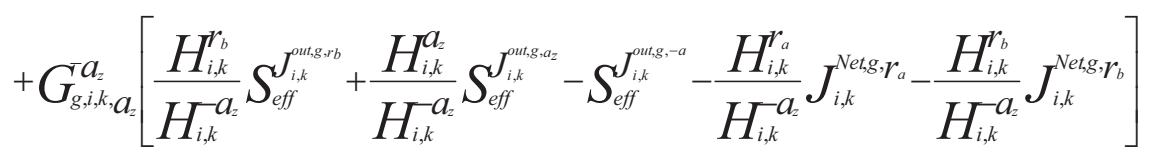

$$
\begin{aligned}
& -G_{g, i, k, a_{z}}^{a_{z}}\left[\frac{H_{i, k}^{a_{2}}}{H_{i, k}^{a_{z}}} J_{i, k}^{\mathrm{Netg}, a_{z}}+\frac{1}{H_{i, k}^{a_{z}}} \Phi_{i, k}\right]=G_{g, i, k+1,-a_{z}}^{a_{a}} J_{i, k+1}^{\mathrm{Netg}, r_{a}}-G_{g, i, k+1,-a_{z}}^{r_{b}} J_{i, k+1}^{\mathrm{Netg}, r_{b}} \\
& =G_{g, i, k+1,-a_{z}}^{{ }_{a}} J_{i, k+1}^{\text {Net, }, r_{a}}-G_{g, i, k+1,-a_{z}}^{r_{b}} J_{i, k+1}^{\text {Net, }, r_{b}}
\end{aligned}
$$

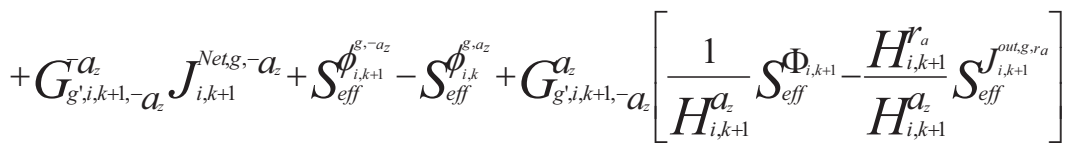

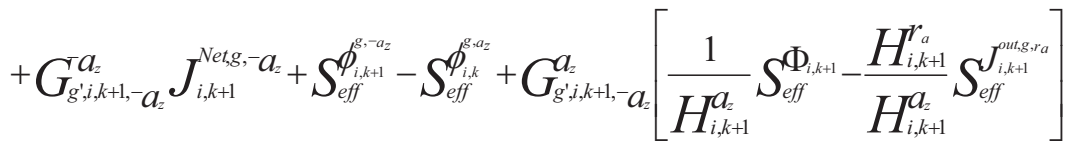

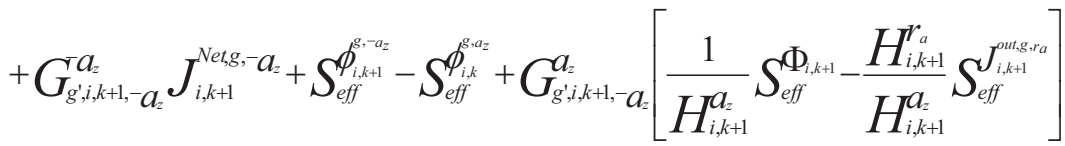

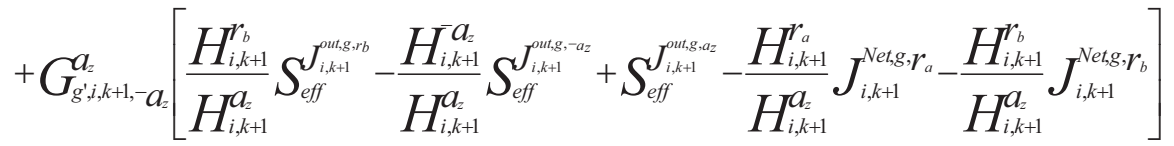

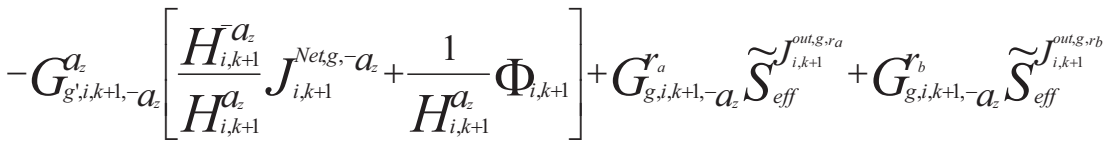

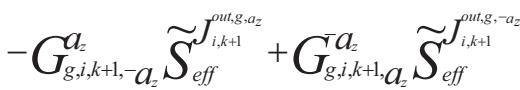


The net current at the top of the node $(\mathrm{i}, \mathrm{k})$ is the same net current at the bottom of the node $(\mathrm{i}, \mathrm{k}+1)$. Algebraically collecting common interface net current and other like terms yields

$$
\begin{aligned}
& \bar{J}^{\text {Netg }}=\frac{G_{g, i, k, a_{z}}^{a_{z}}}{\tau_{k, k+1} H_{i, k}^{-a_{z}}} \Phi_{i, k}-\frac{G_{g, i, k, a_{z}}^{a_{z}}}{\tau_{k, k+1} H_{i, k}^{a_{2}}} S_{e f f}^{\Phi_{i, k}}-\frac{1}{\tau_{k, k+1}}\left[G_{g, i, k, a_{z}}^{a_{a}}-\frac{H_{i, k}^{r_{a}}}{H_{i, k}^{a_{z}}} G_{g, i, k, a_{2}}^{a_{z}}\right] J_{i, k}^{\text {Net, }, r_{a}}
\end{aligned}
$$

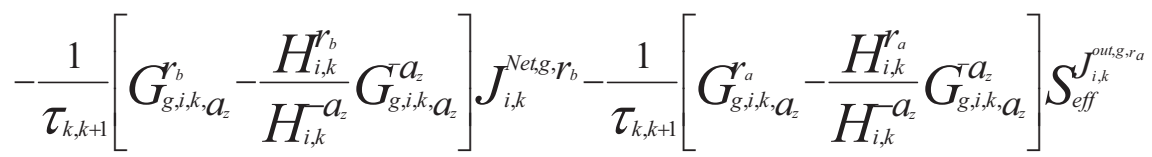

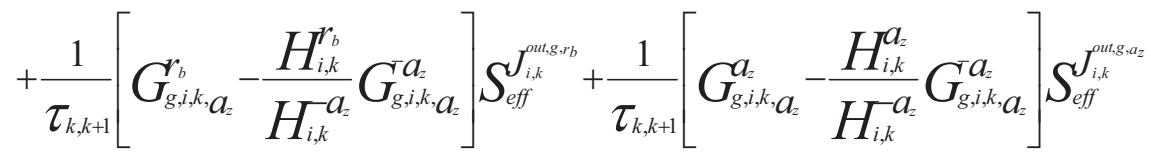

$$
\begin{aligned}
& +\frac{1}{\tau_{k, k+1}}\left[G_{g, i, k+1,-a_{z}}^{r_{a}}-\frac{H_{i, k+1}^{r_{a}}}{H_{i, k+1}^{a_{z}}} G_{g^{\prime}, i, k+1,-a_{z}}^{a_{2}}\right] J_{i, k+1}^{\text {Net, } \boldsymbol{r}_{a}}+\frac{1}{\tau_{k, k+1}}\left[G_{g, i, k+1,-a_{z}}^{r_{b}}-\frac{H_{i, k+1}^{r_{b}}}{H_{i, k+1}^{a_{z}}} G_{g^{\prime}, i, k+1,-a_{z}}^{a_{z}}\right] J_{i, k+1}^{\text {Netg, } \boldsymbol{r}_{b}}
\end{aligned}
$$

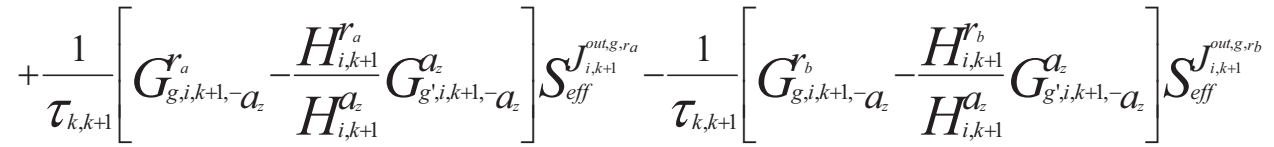

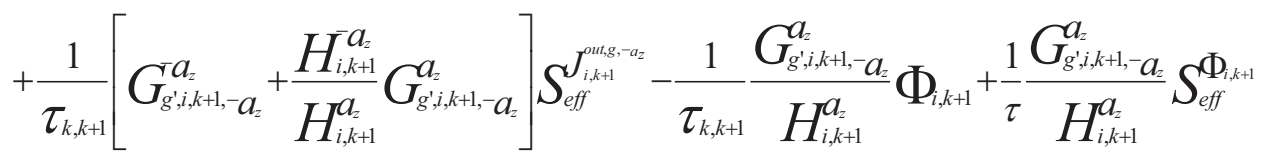

where

$$
\tau_{k, k+1}=G_{g, i, k, a_{z}}^{a_{z}}-G_{g, i, k, a_{z}}^{-a_{z}} \frac{H_{i, k}^{a_{z}}}{H_{i, k}^{-a_{z}}}-G_{g, i, k+1,-a_{z}}^{-a_{z}}+G_{g, i, k+1,-}^{a_{z}} \frac{H_{z, k+1}^{-a_{z}}}{H_{i, k+1}^{a_{z}}}
$$


Equation (68) is the expression for the net current at the boundary top of a response treated node adjoined to another response treated node. A similar expression for the net current can be derived for the lower boundary either by following the algebra above or by shifting the node index for the axial direction. The end result by either method yields

$$
\begin{aligned}
& \bar{J}^{\text {Netg }}=\frac{G_{g, i, k-1, a_{z}}^{a_{z}}}{\tau_{k, k-1} H_{i, k-1}^{-a}} \Phi_{i, k-1}-\frac{G_{g, i, k-1,}^{a_{z}} a_{z}}{\tau_{k, k-1} H_{i, k-1}^{a_{z}}} S_{e f f}^{\Phi_{i, k}}-\frac{1}{\tau_{k, k-1}}\left[G_{g, i, k-1, a_{z}}^{r_{a}}-\frac{H_{i, k-1}^{r_{a}}}{H_{i, k-1}^{-a_{z}}} G_{g, i, k-1, a_{z}}^{a_{z}}\right] J_{i, k-1}^{\text {Net, }, r_{a}} \\
& -\frac{1}{\tau_{k, k-1}}\left[G_{g, i, k-1, a_{z}}^{b_{b}}-\frac{H_{i, k-1}^{r_{b}}}{H_{i, k-1}^{-a_{z}}} G_{g, i, k-1, a_{z}}^{a_{z}}\right] J_{i, k-1}^{N e t g, r_{b}}-\frac{1}{\tau_{k, k-1}}\left[G_{g, i, k-1, a_{z}}^{a_{a}}-\frac{H_{i, k-1}^{r_{a}}}{H_{i, k-1}^{-a_{z}}} G_{g, i, k-1, a_{z}}^{a_{z}}\right] S_{e f f}^{J_{i, k-1}^{\text {out, , r a }}}
\end{aligned}
$$

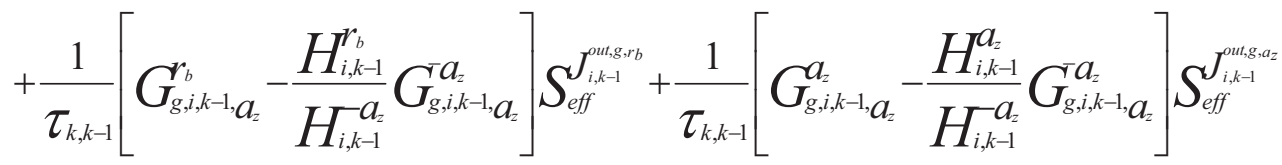

$$
\begin{aligned}
& +\frac{1}{\tau_{k, k-1}}\left[G_{g, i, k,-}^{a} a_{z}-\frac{H_{i, k}^{r_{a}}}{H_{i, k}^{a_{z}}} G_{g^{\prime}, i, k,-}^{a_{z}}\right] J_{i, k}^{N e t, g, r_{a}}+\frac{1}{\tau_{k, k-1}}\left[G_{g, i, k,-}^{b_{z}}-\frac{H_{i, k}^{r_{b}}}{H_{i, k}^{a_{z}}} G_{g^{\prime}, i, k,-}^{a_{z}}\right] J_{i, k}^{\text {Net,g, } r_{b}}
\end{aligned}
$$

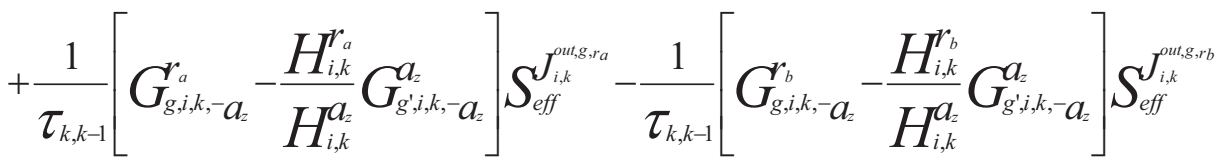

$$
\begin{aligned}
& +\frac{1}{\tau_{k, k-1}}\left[G_{g^{\prime}, i, k,-a_{z}}^{a_{z}}+\frac{H_{i, k}^{a_{z}}}{H_{i, k}^{a_{z}}} G_{g^{\prime}, i, k,-}^{a_{z}}\right] S_{e f f}^{J_{i, k}^{o u, g,-a_{z}}}-\frac{1}{\tau_{k, k-1}} \frac{G_{g^{\prime}, i, k,-}^{a_{z}}}{H_{i, k}^{a_{z}}} \Phi_{i, k}+\frac{1}{\tau_{k, k-1}} \frac{G_{g^{\prime}, i, k,-}^{a_{z}}}{H_{i, k}^{a_{z}}} S_{e f f}^{\Phi_{i, k}}
\end{aligned}
$$

where

$$
\tau_{k, k-1}=G_{g, i, k-1, a_{z}}^{a_{z}}-G_{g, i, k-1, a_{z}}^{-a_{z}} \frac{H_{i, k-1}^{a_{z}}}{H_{i, k-1}^{-a_{z}}}-G_{g, i, k,-a_{z}}^{-a_{z}}+G_{g, i, k,-a_{z}}^{a_{z}} \frac{H_{i, k}^{-a_{z}}}{H_{i, k}^{a_{z}}}
$$

\subsection{Common Interface Current for a Response Node Adjoining a Diffusion Node on the Axial Boundary}

The expression for the interface net current of a pair of nodes is derived in this section. One of the nodes is identified as a diffusion node while the neighboring node is identified as a transport or response node. The diffusion node is treated by the Green's function solution approach while the response node is treated with a method based on the use of response functions.

The derivation of the equation for the interface net current between two nodes starts with the statement of the continuity condition of the scalar flux at the interface between the nodes. (When discontinuity factors are available, the continuity condition is replaced by the appropriate discontinuity condition.) The equations for the scalar node-edge fluxes of a node are substituted into the expression that states the interface flux continuity for that node and its adjacent one. The diffusion side scalar flux is expressed using the diffusion Green's function formulation, and the response node side scalar flux is given as the response to incoming currents. The incoming currents for the response side are then replaced with expressions in terms of net currents. This procedure results in an equation that contains net currents and sources. The net currents for the opposite boundaries (those away from the common interface) are 
then replaced with expressions obtained from the balance equation over each node. This substitution yields an expression that contains the common interface current, the average flux each node, the adjacent interface currents, and the effective sources of each node. After some algebra, the common interface net current between a diffusion node and a response node is obtained.

There are two situations in which a response node and a diffusion node are adjacent to one another in the axial direction: the response node is above the diffusion node, or the other way around, with the response node below the diffusion node. In each situation, the flux at the interface between the two nodes requires a departure from the classic statement of continuity or the discontinuity that results from modern equivalence theory. The new discontinuity equation that is required allows for a discontinuity factor only on the diffusion side of the interface. The continuity condition (or discontinuity condition) for a diffusion treated node above a response function treated node is

$$
f_{i, k+1}^{b} \phi^{\text {diffusion }}=\phi^{\text {tr }}
$$

The factor multiplying the flux on the diffusion side is a discontinuity factor. The derivation and theory of these factors are well known and are not detailed here. The index b given in Equation (70) stands for the bottom discontinuity factor. The continuity condition when the response node is above a diffusion node is

$$
f_{i, k-1}^{t} \phi^{\text {diffusion }}=\phi^{t r}
$$

The index $t$ used in Equation (71) stands for the top discontinuity factor. Equations for the scalar flux at the common interface boundary are needed to obtain the common interface net current. Equations (51) and (52) are the needed expressions for the scalar flux at the boundary of a response node. A Green's function formulation of the scalar flux for the top boundary of a diffusion node is

$$
\phi_{i, k}^{a_{z}}=G\left(a_{z},-a_{z}\right) J_{i, k}^{N e t, g,-a_{z}}-G\left(a_{z}, a_{z}\right) J_{i, k}^{N e t, g, a_{z}}+\int_{-a_{z}}^{a_{z}} G\left(a_{z}, z^{\prime}\right) S_{0}\left(z^{\prime}\right) d z^{\prime}
$$

and the Green's function formulation of the scalar flux for the bottom boundary of the diffusion node is

$$
\phi_{i, k}^{-a_{z}}=G\left(-a_{z},-a_{z}\right) J_{i, k}^{N e t, g,-a_{z}}-G\left(-a_{z}, a_{z}\right) J_{i, k}^{N e t, g, a_{z}}+\int_{-a_{z}}^{a_{z}} G\left(-a_{z}, z^{\prime}\right) S_{0}\left(z^{\prime}\right) d z^{\prime}
$$

In Equations (72) and (73), the top boundary of the node is denoted $a_{z}$ and the bottom boundary of the node is denoted by $-a_{z}$. The node width is, therefore, equal to $2 a_{z}$. Again the index $i$ is the radial node spatial index and the index $\mathrm{k}$ is the axial node spatial index. The index $g$ denotes the current energy group. The integral term is the source term for the diffusion treated node. The source term includes the transverse leakage, the down-scatter into group $g$, the up-scatter into group $g$, and the fission sources into group $g$. The function multiplying the net boundary currents and integrated with the source terms is the axial Green's function. The derivation of the numerical solution of the diffusion equation with a nodal Green's function approach is well $\mathrm{known}^{3,4}$ and is not shown here.

Replacing the scalar fluxes in the continuity (or discontinuity) equation, Equation (70), by its respective expressions from Equations (73) and (53), respectively, there results 


$$
\begin{gathered}
f_{i, k+1}^{b}\left[G\left(-a_{z, i, k+1},-a_{z, i, k+1}\right) J_{i, k+1}^{N e t, g,-a_{z}}-G\left(-a_{z, i, k+1}, a_{z, i, k+1}\right) J_{i, k+1}^{N e t, g, a_{z}}+\int_{-a_{z, i, k+1}}^{a_{z, i, k+1}} G\left(-a_{z, i, k+1}, z^{\prime}\right) S_{0}\left(z^{\prime}\right) d z^{\prime}\right] \\
=R_{g \rightarrow g, r_{a} \rightarrow a_{z}}^{\phi_{i, k}^{a_{z}}} J_{i, k}^{i,, g, r_{a}}+R_{g \rightarrow g, r_{b} \rightarrow a_{z}}^{\phi_{i, k}^{a_{z}}} J_{i, k}^{i, g, r_{b}}+R_{g \rightarrow g, a_{z} \rightarrow a_{z}}^{\phi_{i, k}^{a_{z}}} J_{i, k}^{i, g, a_{z}}+R_{g \rightarrow g,-a_{z} \rightarrow a_{z}}^{\phi_{i, k}^{a_{z}}} J_{i, k}^{i, g,-a_{z}}+\widetilde{S}_{e f f}^{\phi_{i, k}^{a_{z}}}
\end{gathered}
$$

Equation (74) expresses the continuity (or discontinuity) condition of the scalar flux as a combination of net currents and incoming partial currents. However, an expression is sought that involves only interface net currents. Thus Equations (41), (42), (45), and (46) are substituted into Equation (74). The terms for the net currents and sources are then grouped; the result is

$$
\begin{aligned}
& f_{i, k+1}^{b} G\left(-a_{z, i, k+1},-a_{z, i, k+1}\right) J_{i, k+1}^{N e t, g,-a_{z}}-f_{i, k+1}^{b} G\left(-a_{z, i, k+1}, a_{z, i, k+1}\right) J_{i, k+1}^{N e t, g} a_{z}+f_{i, k+1}^{b} \int_{-a_{z}}^{a_{z}} G\left(-a_{z, i, k+1}, z^{\prime}\right) S_{0}\left(z^{\prime}\right) d z^{\prime} \\
& =G_{g, i, k, a_{z}}^{a_{z}} J_{i, k}^{\text {Netg, } r_{a}}+G_{g, i, k}^{b} a_{z} J_{i, k}^{\text {Netg, } r_{b}}+G_{g, i, k, a_{z}}^{a_{z}} J_{i, k}^{\text {Net,g, } a_{z}} \\
& +G_{g, i, k, a_{z}}^{a_{z}} J_{i, k}^{\text {Netg, }, a_{z}}+\widetilde{S}_{e f f}^{\phi_{i, k}^{a_{z}}}+G_{g, i, k, a_{z}}^{r_{a}} \widetilde{S}_{\text {eff }}^{J_{i, k}^{\text {out, r,a }}} \\
& -G_{g, i, k, a_{z}}^{b_{b}} \widetilde{S}_{e f f}^{J_{i, k}^{o u, g, r b}}-G_{g, i, k, a_{z}}^{a_{z}} \widetilde{S}_{e f f}^{J_{i, k}^{o u, g, a_{z}}}+G_{g, i, k, a_{z}}^{a_{z}} \widetilde{S}_{e f f}^{J_{i, k}^{o u, g,-a_{z}}}
\end{aligned}
$$

The definition of the coefficients in front of the net currents and the effective sources on the side of the response node are given by Equations (59), (60), (61), and (62). Next, expressions for the opposite face net currents are derived from the nodal balance equation and substituted into Equation (75). The expression for the top axial net current for the diffusion node is obtained from the nodal balance equation. However, the expression for the bottom axial current (response node) is derived from Equation (50). The result of the substitutions is an equation that relates the common interface net current to that of the adjacent net currents of the response node, the average scalar flux of both nodes, and effective sources of both nodes. The new equation is

$$
\begin{aligned}
& f_{i, k+1}^{b} G\left(-a_{z, i, k+1},-a_{z, i, k+1}\right) J_{i, k+1}^{N e t, g,-a_{z}} \\
& -f_{i, k+1}^{b} G\left(-a_{z, i, k+1}, a_{z, i, k+1}\right)\left[2 a_{z, i, k+1} \bar{S}_{0}-2 a_{z, i, k+1} \Phi_{i, k+1}+J_{i, k+1}^{N e t, g,-a_{z}}\right] \\
& +f_{i, k+1}^{b} \int_{-a_{z, i, k+1}}^{a_{z, i, k+1}} G\left(-a_{z, i, k+1}, z^{\prime}\right) S_{0}\left(z^{\prime}\right) d z \\
& =G_{g, i, k, a_{z}}^{r_{a}} J_{i, k}^{N e t, g, r_{a}}+G_{g, i, k, a_{z}}^{r_{b}} J_{i, k}^{\mathrm{Net,g}, r_{b}}+G_{g, i, k, a_{z}}^{a_{z}} J_{i, k}^{\text {Net,g, } a_{z}} \\
& +G_{g, i, k, a_{z}}^{a_{z}}\left[\frac{1}{H_{i, k}^{a_{z}}} \widetilde{S}_{e f f}^{\Phi_{i, k}}-\frac{H_{i, k}^{a_{z}}}{H_{i, k}^{a_{z}}} J_{i, k}^{\mathrm{Net,g,}, a_{z}}-\frac{H_{i, k}^{r_{a}}}{H_{i, k}^{a}} J_{i, k}^{\mathrm{Net,g,r_{a }}}-\frac{H_{i, k}^{r_{b}}}{H_{i, k}^{a_{z}}} J_{i, k}^{N e t, g, r_{b}}-\frac{1}{H_{i, k}^{a_{z}}} \Phi_{i, k}\right] \\
& +G_{g, i, k, a_{z}}^{a_{z}}\left[\frac{H_{i, k}^{a_{z}}}{H_{i, k}^{a_{z}}} \widetilde{S}_{\text {eff }}^{J_{i, k}^{\text {out,g,az}}}-\frac{H_{i, k}^{a_{z}}}{H_{i, k}^{a_{z}}} \widetilde{S}_{\text {eff }}^{J_{i, k}^{\text {out, },-a_{z}}}-\frac{H_{i, k}^{r_{a}}}{H_{i, k}^{a_{z}}} \widetilde{S}_{\text {eff }}^{J_{i, k}^{\text {out,g,ra }}}+\frac{H_{i, k}^{r_{b}}}{H_{i, k}^{a_{z}}} \widetilde{S}_{\text {eff }}^{J_{i, k}^{\text {out,g,rb}}}\right]
\end{aligned}
$$

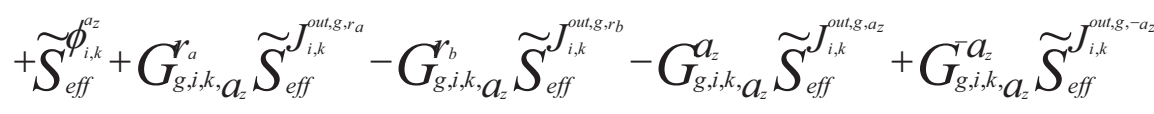


In Equation (76), the transverse leakage terms for the node with the diffusion treatment are incorporated into the source terms, but the transverse leakage net currents for the response node are expressed explicitly. Next, Equation (76) is solved for the common interface net current. The equation for the common interface current for a diffusion treated node above a response treated node is

$$
\begin{aligned}
& J^{N e t}=-\frac{f_{i, k+1}^{b}}{\tau_{k, k+1}} \int_{-a_{z, i, k+1}}^{a_{z, i, k+1}} G\left(-a_{z, i, k+1}, z^{\prime}\right) S_{0}\left(z^{\prime}\right) d z^{\prime}+\frac{2 a_{z, i, k+1} f_{i, k+1}^{b}}{\tau_{k, k+1}} G\left(-a_{z, i, k+1}, a_{z, i, k+1}\right) \bar{S}_{0} \\
& +\frac{2 a_{z, i, k+1} f_{i, k+1}^{b}}{\tau_{k, k+1}} G\left(-a_{z, i, k+1}, a_{z, i, k+1}\right) \Phi_{i, k+1}-\frac{1}{\tau_{k, k+1}} \frac{G_{g, i, k,}^{-a_{z}}}{H_{i, k}^{-a_{z}}} \Phi_{i, k}+\frac{1}{\tau_{k, k+1}} \frac{G_{g, i, k, a_{z}}^{-a_{z}}}{H_{i, k}^{-} a_{z}} \widetilde{S}_{e f f}^{\Phi_{i, k}} \\
& +\frac{1}{\tau_{k, k+1}}\left[G_{g, i, k, a_{z}}^{r_{a}}-\frac{H_{i, k}^{r_{a}}}{H_{i, k}^{-a_{z}}} G_{g, i, k, a_{z}}^{-a_{z}}\right] J_{i, k}^{N e t, g, r_{a}}+\frac{1}{\tau_{k, k+1}}\left[G_{g, i, k, a_{z}}^{r_{b}}-\frac{H_{i, k}^{r_{b}}}{H_{i, k}^{-a_{z}}} G_{g, i, k, a_{z}}^{-a_{z}}\right] J_{i, k}^{N e t, g, \boldsymbol{r}_{b}} \\
& +\frac{1}{\tau_{k, k+1}}\left[G_{g, i, k, a_{z}}^{r_{a}}-\frac{H_{i, k}^{r_{a}}}{H_{i, k}^{-} a_{z}} G_{g, i, k, a_{z}}^{-a_{z}}\right] \widetilde{S}_{\text {eff }}^{J_{i, k}^{\text {out,g,ra }}}-\frac{1}{\tau_{k, k+1}}\left[G_{g, i, k, a_{z}}^{r_{b}}-\frac{H_{i, k}^{r_{b}}}{H_{i, k}^{-} a_{z}} G_{g, i, k, a_{z}}^{-a_{z}}\right] \widetilde{S}_{e f f}^{J_{i, k}^{\text {out, },, r b}} \\
& -\frac{1}{\tau_{k, k+1}}\left[G_{g, i, k, a_{z}}^{a_{z}}-\frac{H_{i, k}^{a_{z}}}{H_{i, k}^{-a_{z}}} G_{g, i, k, a_{z}}^{-a_{z}}\right] \widetilde{S}_{e f f}^{J_{i, k}^{o u t, g, a_{z}}}+\frac{1}{\tau_{k, k+1}} \widetilde{S}_{e f f}^{\phi_{i, k}^{a_{z}}}
\end{aligned}
$$

where

$$
\tau_{k, k+1}=f_{i, k+1}^{b} G\left(-a_{z, i, k+1},-a_{z, i, k+1}\right)-f_{i, k+1}^{b} G\left(a_{z, i, k+1},-a_{z, i, k+1}\right)-G_{g, i, k, a_{z}}^{a_{z}}+\frac{H_{i, k}^{a_{z}}}{H_{i, k}^{-} a_{z}} G_{g, i, k, a_{z}}^{-} a_{z}
$$

Equation (77) expresses the net current at the interface between a node treated with diffusion and a node treated with response functions. Equation (77) relates the net current at the interface between the two adjacent nodes to the average fluxes of each node and the sources, which include the transverse leakage terms. 
A similar equation can be derived for the interface net current when the node treated with response functions is above a node treated with diffusion. The derivation of the equation for the net current in this situation is similar to the derivation just described for the case when the response node is below the diffusion node. The equation for the interface net current between an upper response node and a lower diffusion node is

$$
\begin{aligned}
& J^{N e t}=-\frac{f_{i, k-1}^{t}}{\tau_{k, k-1}} \int_{-a_{z, i, k-1}}^{a_{z, i, k-1}} G\left(a_{z, i, k-1}, z^{\prime}\right) S_{0}\left(z^{\prime}\right) d z^{\prime}+\frac{2 a_{z, i, k-1} f_{i, k-1}^{t}}{\tau_{k, k-1}} G\left(-a_{z, i, k-1}, a_{z, i, k-1}\right) \bar{S}_{0} \\
& +\frac{2 a_{z, i, k-1} f_{i, k-1}^{t}}{\tau_{k, k-1}} G\left(-a_{z, i, k-1}, a_{z, i, k-1}\right) \Phi_{i, k-1}-\frac{1}{\tau_{k, k-1}} \frac{G_{g, i, k,-}^{a_{z}}}{H_{i, k}^{a_{z}}} \Phi_{i, k}+\frac{1}{\tau_{k, k-1}} \frac{G_{g, i, k,-}^{a_{z}}}{H_{i, k}^{a_{z}}} \widetilde{S}_{e f f}^{\Phi_{i, k}} \\
& +\frac{1}{\tau_{k, k-1}}\left[G_{g, i, k,-}^{r_{a}}-\frac{H_{i, k}^{r_{a}}}{H_{i, k}^{a_{z}}} G_{g, i, k,-a_{z}}^{a_{z}}\right] J_{i, k}^{N e t, g, r_{a}}+\frac{1}{\tau_{k, k-1}}\left[G_{g, i, k,-}^{r_{b}}-\frac{H_{i, k}^{r_{b}}}{H_{i, k}^{a_{z}}} G_{g, i, k,-a_{z}}^{a_{z}}\right] J_{i, k}^{\text {Net,g, } r_{b}} \\
& +\frac{1}{\tau_{k, k-1}}\left[G_{g, i, k,-a_{z}}^{r_{a}}-\frac{H_{i, k}^{r_{a}}}{H_{i, k}^{a_{z}}} G_{g, i, k,-}^{a_{z}}\right] \widetilde{S}_{\text {eff }}^{J_{i, k}^{\text {out,g,ra}}}-\frac{1}{\tau_{k, k-1}}\left[G_{g, i, k,-}^{r_{b}}-\frac{H_{i, k}^{r_{b}}}{H_{i, k}^{a_{z}}} G_{g, i, k,-}^{a_{z}}\right] \widetilde{S}_{\text {eff }}^{J_{i, k}^{\text {out, }, r, b}} \\
& +\frac{1}{\tau_{k, k-1}}\left[G_{g, i, k,-a_{z}}^{-a_{z}}-\frac{H_{i, k}^{-a_{z}}}{H_{i, k}^{a_{z}}} G_{g, i, k,-a_{z}}^{a_{z}}\right] \widetilde{S}_{e f f}^{J_{i, k}^{\text {out, }, a_{z}}}+\frac{1}{\tau_{k, k-1}} \widetilde{S}_{e f f}^{\phi_{i, k}^{a_{z}}}
\end{aligned}
$$

where

$$
\tau_{k, k-1}=f_{i, k-1}^{t} G\left(-a_{z, i, k-1}, a_{z, i, k-1}\right)-f_{i, k-1}^{t} G\left(a_{z, i, k-1}, a_{z, i, k-1}\right)-G_{g, i, k, a_{z}}^{a_{z}}+\frac{H_{i, k}^{-a_{z}}}{H_{i, k}^{a_{z}}} G_{g, i, k,-a_{z}}^{a_{z}}
$$

\subsection{Common Interface Current for a Response Node Adjoining an Axial Boundary}

The net current for a response node at the boundary of a reactor is derived in this section. To begin the derivation, the notation for the boundary condition is first established, then the flux at the boundary is matched to a response function expression for the boundary scalar flux. The result is an equation that contains incoming currents, sources, and net currents. The incoming partial currents are replaced by equations containing net currents and effective sources. The result of the substitution is an equation that includes the incoming current (as a source), the net currents, and effective sources. The net current at the node boundary opposite the reactor boundary is replaced by an equation that contains average fluxes, adjacent net currents, the reactor boundary net current, and effective sources for the node. The elimination of the opposite net current gives an expression that is then manipulated to obtain the net current along the reactor boundary.

The derivation of an equation for the net current at the reactor boundary starts with the statement of the boundary conditions. The equation for the boundary condition at the top of the reactor is

$$
J^{i n,-a_{z}}=\alpha_{-a_{z}} \phi^{-a_{z}}+\beta_{-a_{z}} J_{g}^{N e t,-a_{z}}
$$


and the equation for the bottom boundary condition is

$$
J^{i n,-a_{z}}=\alpha_{-a_{z}} \phi^{-a_{z}}+\beta_{-a_{z}} J_{g}^{N e t,-a_{z}}
$$

Equations (79) and (80) express the incoming current in terms of the scalar flux and the net current at the reactor boundary. They are a mathematical means of representing the rest of the universe beyond the reactor boundary. In this respect, the boundary condition can be coupled to the corresponding equation within the boundary node to derive expressions for boundary quantities in the same way equations within two adjacent nodes within the reactor can be used together to derive expressions for quantities on their mutual interface.

In Equations (79) and (80), the coefficients, $\alpha_{-\mathrm{az}}, \beta_{-\mathrm{az}}, \alpha_{\mathrm{az}}, \beta_{\mathrm{az}}$ and the incoming currents are part of the specified input conditions. For a boundary condition where linear anisotropy is assumed, the coefficients $\alpha_{-\mathrm{az}}$ and $\alpha_{\mathrm{az}}$ are set equal to 0.25 , the coefficient $\beta_{-\mathrm{az}}$ is set equal to -0.5 , and the coefficient $\beta_{\mathrm{az}}$ is set equal to 0.5 .

Consider a boundary node at the top of the reactor that is treated through the response function approach. An expression must be obtained for the outer boundary net current of said reactor boundary node. For this, the scalar flux in the expression of the boundary condition is set equal to the scalar flux that is obtained as the node surface flux response at that same (boundary) surface. This means that the edge scalar flux in Equation (79) is set equal to the edge scalar flux in Equation (53). When dealing with a response boundary node at the bottom of the reactor, the same procedure involves equating the scalar surface boundary fluxes from Equations (80) and (54).

For a top-of-reactor boundary node, the process gives

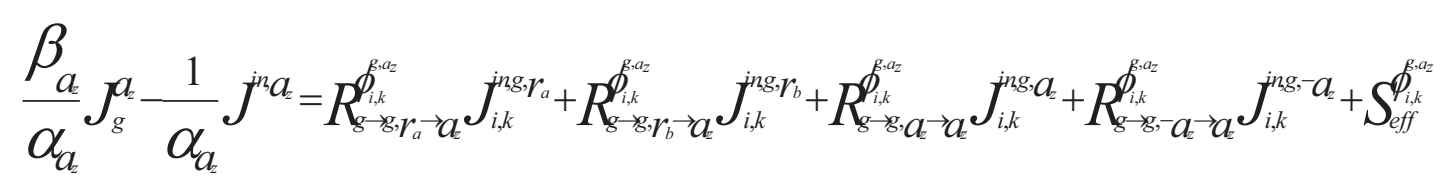

Equations (41), (42), (45), and (46) are introduced into Equation (81), replacing the four partial currents that appear multiplied by axial boundary scalar flux response functions. Although the incoming partial current for the surface at $\mathrm{a}_{\mathrm{z}}$ that appears on the right side of Equation (81) may be specified as part of the boundary condition, the incoming current on side $\mathrm{a}_{\mathrm{z}}$ is replaced by its expression from the relevant equation, as just stated. The resulting equation is

$$
\begin{aligned}
& \frac{\beta_{a_{z}}}{\alpha_{a_{z}}} J_{g}^{N e t, g, a_{z}}-\frac{1}{\alpha_{a_{z}}} J^{i n, a_{z}}=G_{g, i, k, a_{z}}^{a^{a}} J_{i, k}^{N e t, g, r_{a}}+G_{g, i, k, a_{z}}^{r_{b}} J_{i, k}^{\text {Net,g, } r_{b}}+G_{g, i, k, a_{z}}^{a_{z}} J_{i, k}^{N e t, g, a_{z}}+G_{g, i, k, a_{z}}^{a_{z}} J_{i, k}^{N e t, g,-a_{z}}
\end{aligned}
$$

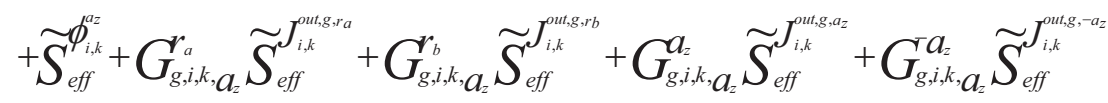


The coefficients in front of the net currents on the right side of Equation (82) are given by Equations (59), (60), (61), and (62). Next, the expression for the net current at the bottom edge of the boundary node is substituted into Equation (82). The substitution leaves an equation that retains only the net current at the external (top) surface of the node and currents in the transverse direction (the r-direction). The resulting equation is

$$
\begin{aligned}
& \frac{\beta_{a_{z}}}{\alpha_{a_{z}}} J_{g}^{\text {Net, }, a_{z}}-\frac{1}{\alpha_{a_{z}}} J^{i n \cdot a_{z}} \\
& =G_{g, i, k, a_{z}}^{r_{a}} J_{i, k}^{\text {Net,g, } r_{a}}+G_{g, i, k, a_{z}}^{b_{b}} J_{i, k}^{\text {Net,g, } r_{b}}+G_{g, i, k, a_{z}}^{a_{z}} J_{i, k}^{\text {Netg, } a_{z}} \\
& +G_{g, i, k, a_{z}}^{a_{z}}\left[-\frac{H_{i, k}^{g, r_{a}}}{H_{i, k}^{g,-a_{z}}} J_{i, k}^{\mathrm{Net,g}, \boldsymbol{r}_{a}}-\frac{H_{i, k}^{g, r_{b}}}{H_{i, k}^{g,-a_{z}}} J_{i, k}^{\mathrm{Net,g}, \boldsymbol{r}_{b}}-\frac{H_{i, k}^{g, a_{z}}}{H_{i, k}^{g,-a_{z}}} J_{i, k}^{\mathrm{Net,g}, a_{z}}-\frac{1}{H_{i, k}^{g,-a_{z}}} \Phi^{g}\right]
\end{aligned}
$$

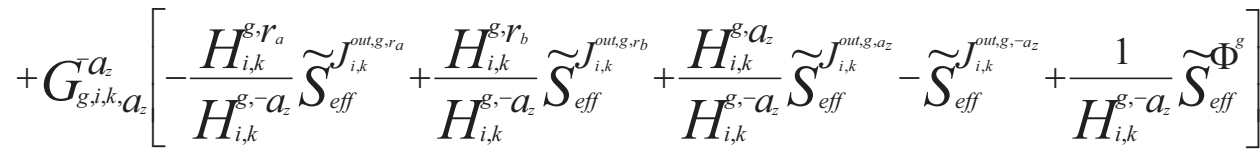

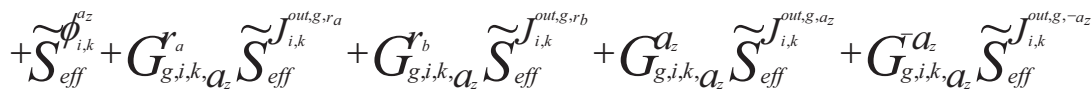

Upon rearranging its terms, Equation (83) is solved for the outer edge net current, thus providing an expression for the net current on the reactor boundary,

$$
\begin{aligned}
& J^{N e t}=\frac{1}{\tau_{k, k+1}} \frac{1}{\alpha_{a_{z}}} J^{i n, a_{z}}-\frac{1}{\tau_{k, k+1}} \frac{G_{g, i, k, a_{z}}^{-a_{z}}}{H_{i, k}^{-a_{z}}} \Phi_{i, k}+\frac{1}{\tau_{k, k+1}} \frac{G_{g, i, k, a_{z}}^{-a_{z}}}{H_{i, k}^{-} a_{z}} \widetilde{S}_{e f f}^{\Phi_{i, k}} \\
& +\frac{1}{\tau_{k, k+1}}\left[G_{g, i, k, a_{z}}^{r_{a}}-\frac{H_{i, k}^{r_{a}}}{H_{i, k}^{-a_{z}}} G_{g, i, k, a_{z}}^{-a_{z}}\right] J_{i, k}^{\text {Net, }, r_{a}}+\frac{1}{\tau_{k, k+1}}\left[G_{g, i, k, a_{z}}^{r_{b}}-\frac{H_{i, k}^{r_{b}}}{H_{i, k}^{-a_{z}}} G_{g, i, k, a_{z}}^{-a_{z}}\right] J_{i, k}^{\text {Net, }, g, r_{b}} \\
& +\frac{1}{\tau_{k, k+1}}\left[G_{g, i, k, a_{z}}^{r_{a}}-\frac{H_{i, k}^{r_{a}}}{H_{i, k}^{-a_{z}}} G_{g, i, k, a_{z}}^{-a_{z}}\right] \widetilde{S}_{e f f}^{J_{i, k}^{o u, g, r_{a}}}-\frac{1}{\tau_{k, k+1}}\left[G_{g, i, k, a_{z}}^{r_{b}}-\frac{H_{i, k}^{r_{b}}}{H_{i, k}^{-a_{z}}} G_{g, i, k, a_{z}}^{-a_{z}}\right] \widetilde{S}_{e f f}^{J_{i, k}^{o u, t, r, b}} \\
& -\frac{1}{\tau_{k, k+1}}\left[G_{g, i, k, a_{z}}^{a_{z}}-\frac{H_{i, k}^{a_{z}}}{H_{i, k}^{-a_{z}}} G_{g, i, k, a_{z}}^{-a_{z}}\right] \widetilde{S}_{e f f}^{J_{i, k}^{\text {out, g,az }}}+\frac{1}{\tau_{k, k+1}} \widetilde{S}_{e f f}^{\phi_{i, k}^{a_{z}}}
\end{aligned}
$$

where

$$
\tau_{k, k+1}=\frac{\beta_{a_{z}}}{\alpha_{a_{z}}}-G_{g, i, k, a_{z}}^{a_{z}}+\frac{H_{i, k}^{a_{z}}}{H_{i, k}^{-a_{z}}} G_{g, i, k, a_{z}}^{-a_{z}}
$$


By repeating, for the bottom boundary node, the derivation just shown for the top axial boundary, the equation for the net current at the bottom reactor boundary is derived. The equation for the net current at the bottom boundary is

$$
\begin{aligned}
& J^{N e t}=\frac{1}{\tau_{k, k-1}} \frac{1}{\alpha_{a_{z}}} J^{i n, a_{z}}-\frac{1}{\tau_{k, k-1}} \frac{G_{g, i, k,-}^{a_{z}}}{H_{i, k}^{a_{z}}} \Phi_{i, k}+\frac{1}{\tau_{k, k-1}} \frac{G_{g, i, k,-}^{a_{z}}}{H_{i, k}^{a_{z}}} \widetilde{S}_{e f f}^{\Phi_{i, k}} \\
& +\frac{1}{\tau_{k, k-1}}\left[G_{g, i, k,-a_{z}}^{r_{a}}-\frac{H_{i, k}^{r_{a}}}{H_{i, k}^{a_{z}}} G_{g, i, k,-a_{z}}^{a_{z}}\right] J_{i, k}^{N e t, g, r_{a}}+\frac{1}{\tau_{k, k-1}}\left[G_{g, i, k,-a_{z}}^{r_{b}}-\frac{H_{i, k}^{r_{b}}}{H_{i, k}^{a_{z}}} G_{g, i, k,-a_{z}}^{a_{z}}\right] J_{i, k}^{\mathrm{Net,g,g}, \boldsymbol{r}_{b}}
\end{aligned}
$$

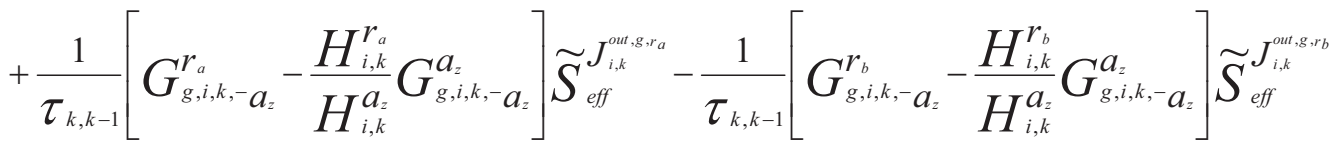

$$
\begin{aligned}
& +\frac{1}{\tau_{k, k-1}}\left[G_{g, i, k,-a_{z}}^{a_{z}}-\frac{H_{i, k}^{-a_{z}}}{H_{i, k}^{a_{z}}} G_{g, i, k,-a_{z}}^{a_{z}}\right] \widetilde{S}_{e f f}^{J_{i, k}^{o u, g, a z}}+\frac{1}{\tau_{k, k-1}} \widetilde{S}_{e f f}^{\phi_{i, k}^{a_{z}}}
\end{aligned}
$$

where

$$
\tau_{k, k-1}=\frac{\beta_{a_{z}}}{\alpha_{a_{z}}} G_{g, i, k,-a_{z}}^{-a_{z}}+\frac{H_{i, k}^{-a_{z}}}{H_{i, k}^{a_{z}}} G_{g, i, k,-a_{z}}^{a_{z}} .
$$




\section{COMMON RADIAL INTERFACE NET CURRENT EQUATIONS}

The equations for the radial net currents are derived in this section. The derivation steps and the results are very similar to the derivations for the axial net currents. The equations for the radial net currents are important because they are substituted into an appropriate balance equation to obtain either the three- or five-striped CMFD (nodal) form of the equation for the average scalar flux. They are also important for obtaining the net current on the axial boundary after values for the scalar fluxes are obtained. Derivations are given in this section for the net radial current equations that include three cases: the case when two response nodes are adjacent to each other in the radial direction, the case when a response node is next to a diffusion treated node, and the case when the response node is at the reactor boundary.

\subsection{Common Interface Current for Two Response Nodes With an Adjoining Boundary in the Radial Direction}

An equation is derived in this section for the common interface current for two adjacent response nodes in the radial direction. The derivation starts by setting equal the common boundary fluxes from the two adjacent nodes, and then expanding the boundary fluxes in terms of response functions multiplied by the respective incoming boundary currents. All the incoming currents are replaced by equations relating the incoming currents for a node to the net currents on the node boundary. These equations were derived in the section for the balance equation of a response node. The terms for the net currents are collected, and the net currents for the opposite faces (farthest surfaces) of the pair of nodes are replaced by an expression derived from the balance equation for each response node. The terms with the net current on the adjoining/common boundary are collected and the net current on the adjoining boundary is algebraically obtained.

The derivation starts by obtaining the side (surface) scalar flux as a response to the incoming currents, as obtained from either a numerical calculation or from an analytical derivation. The radial response function equations for the interface scalar flux on the inner and outer edges of the node are

$\phi_{i, k}^{g, r_{a}}=\sum_{g^{\prime}=1}^{G} \sum_{f=r_{a^{\prime}} r_{b^{\prime}} \boldsymbol{a}_{z^{\prime}},-a_{z}} R_{g^{\prime} \rightarrow g, f \rightarrow r_{a}}^{\phi_{i, r_{a}}} J_{i, k}^{i n, g^{\prime}, f}$

and

$$
\phi_{i, k}^{g, r_{b}}=\sum_{g^{\prime}=1}^{G} \sum_{f=r_{a}, r_{b}, a_{z^{\prime}}, a_{z}} R_{g^{\prime} \rightarrow g, f \rightarrow r_{b}}^{\phi_{i, r_{b}}} J_{i, k}^{i n, g^{\prime}, f}
$$

In Equations (86) and (87), the edges of the node are denoted by $r_{a}, r_{b}, a_{z}$ and $-a_{z}$. The energy group index is given by $g$ and the maximum number of energy groups is denoted by $G$. The index i denotes the radial spatial node index and the index $k$ denotes the axial spatial node index. The inner face of the response node is located at the radius $r_{a}$, and the outer face of the response node is located at the radius $r_{b}$. The incoming currents for the energy groups that are not the current energy group $g$ are held constant for the solution of fluxes and currents in group $g$. These terms are effective source terms for the response nodes, and thus Equations (86) and (87) are rewritten as

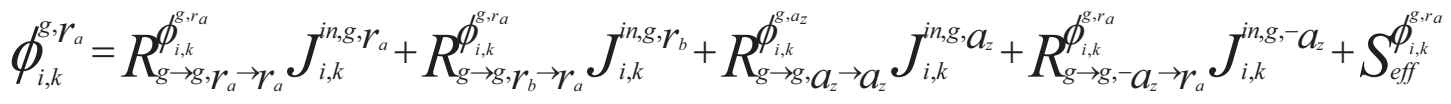

and 
$\phi_{i, k}^{g, r_{b}}=R_{g \rightarrow g, r_{a} \rightarrow r_{b}}^{\phi_{i, r_{b}}} J_{i, k}^{i n, g, r_{a}}+R_{g \rightarrow g, r_{b} \rightarrow r_{b}}^{\phi_{i, k}^{g, r_{b}}} J_{i, k}^{i n, g, r_{b}}+R_{g \rightarrow g, a_{z} \rightarrow r_{b}}^{\phi_{i, k}, r_{b}} J_{i, k}^{i n, g, a_{z}}+R_{g \rightarrow g,-a_{z} \rightarrow r_{b}}^{\phi_{i, k}^{g, r_{b}}} J_{i, k}^{i n, g,-a_{z}}+S_{e f f}^{\phi_{i, k}^{g, r_{b}}}$

where

$$
\widetilde{S}_{e f f}^{\phi_{i, k}^{g, r_{a}}}=\sum_{g^{\prime}=1, g^{\prime} \neq g}^{G} \sum_{f=r_{a}, r_{b^{\prime}} a_{z^{\prime}}-a_{z}} R_{g^{\prime} \rightarrow g, f \rightarrow r_{a}}^{\phi_{i, k}^{g, r_{a}}} J_{i, k}^{i n, g^{\prime}, f}
$$

and

$$
\widetilde{S}_{e f f}^{\phi_{i, k}^{g, r b}}=\sum_{g^{\prime}=1, g^{\prime} \neq g}^{G} \sum_{f=r_{a}, r_{b^{\prime}} \cdot a_{z^{\prime}}-a_{z}} R_{g^{\prime} \rightarrow g, f \rightarrow r_{b}}^{\phi_{i, r_{b}}} J_{i, k}^{i n, g^{\prime}, f}
$$

Equations (90) and (91) are the effective source terms for the scalar flux response equations. Next, at the interface between the response node $(\mathrm{i}, \mathrm{k})$ and $(\mathrm{i}+1, \mathrm{k})$, the expressions for the surface flux from the two nodes are set equal to one another. That is, the expression for the inner radial scalar flux of node $(\mathrm{i}+1, \mathrm{k})$ is set equal to the expression for the outer radial scalar flux of node $(\mathrm{i}, \mathrm{k})$. The resulting equation is

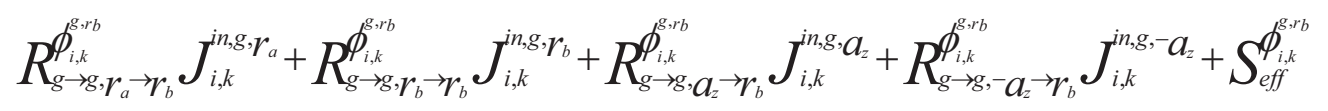

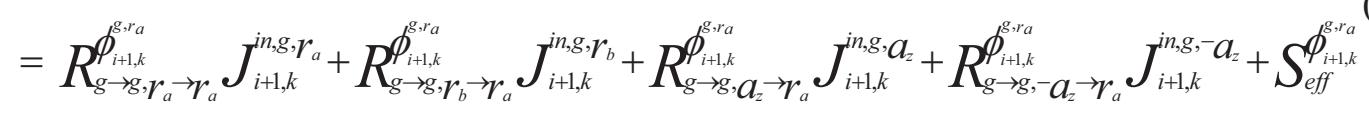

Equations (41), (42), (45), and (46), which express the incoming currents in terms of the net interface currents, are substituted into Equation (92). In the resulting equation, the interface net currents are collected. The equation that results is

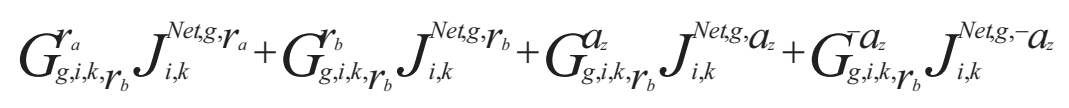

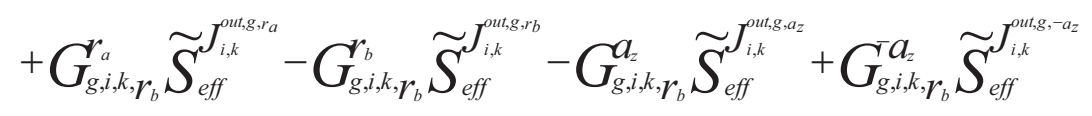

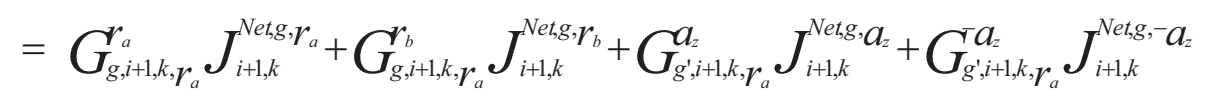

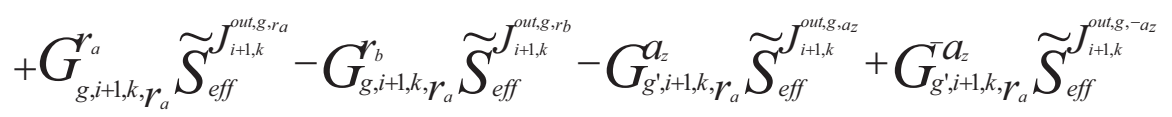

$$
\begin{aligned}
& +S_{e f f}^{\phi_{i+1, k}^{g, r a}}-S_{e f f}^{\phi_{i, k}^{g, r}}
\end{aligned}
$$

The coefficients appearing in front of the net currents are

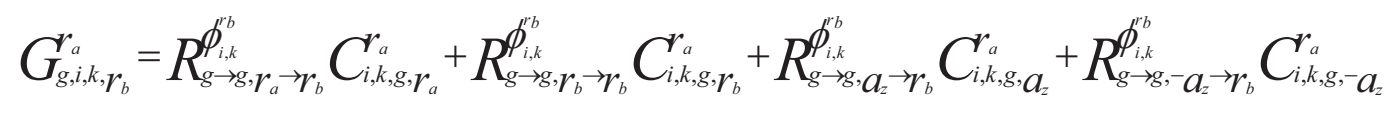

$$
\begin{aligned}
& G_{g, i, k, r_{b}}^{r_{b}}=R_{g \rightarrow g, r_{a} \rightarrow r_{b}}^{\phi_{i, k}^{r_{b}}} C_{i, k, g, r_{a}}^{r_{b}}+R_{g \rightarrow g, r_{b} \rightarrow r_{b}}^{\phi_{i, k}^{r_{b}}} C_{i, k, g, r_{b}}^{r_{b}}+R_{g \rightarrow g, a_{z} \rightarrow r_{b}}^{\phi_{i b, k}^{b_{b}}} C_{i, k, g, a_{z}}^{r_{b}}+R_{g \rightarrow g,-a_{z} \rightarrow r_{b}}^{\phi_{i, k}^{r_{b}}} C_{i, k, g,-a_{z}}^{r_{b}} \\
& G_{g, i, k, r_{b}}^{a_{z}}=R_{g \rightarrow g, r_{a} \rightarrow r_{b}}^{\phi_{i, k}^{r b}} C_{i, k, g, \gamma_{a}}^{a_{z}}+R_{g \rightarrow g, r_{b} \rightarrow r_{b}}^{\phi_{i, k}^{r b}} C_{i, k, g, r_{b}}^{a_{z}}+R_{g \rightarrow g, a_{z} \rightarrow r_{b}}^{\phi_{i, k}^{r b}} C_{i, k, g, a_{z}}^{a_{z}}+R_{g \rightarrow g,-a_{z} \rightarrow r_{b}}^{\phi_{i, k}^{r b}} C_{i, k, g,-a_{z}}^{a_{z}}
\end{aligned}
$$




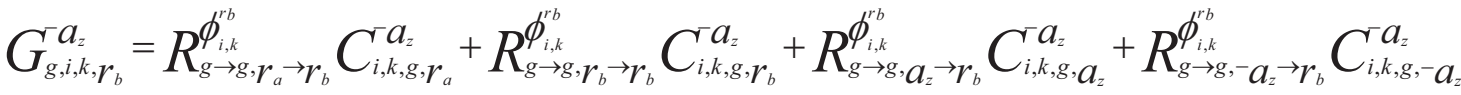

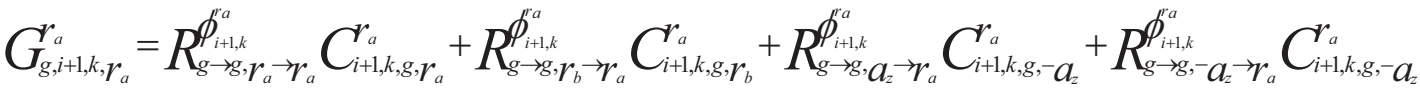

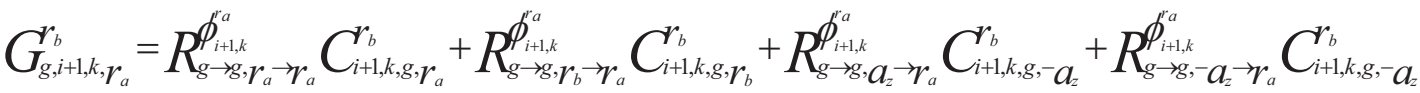

$$
\begin{aligned}
& G_{g, i+1, k, r_{a}}^{a_{z}}=R_{g \rightarrow g, r_{a} \rightarrow \boldsymbol{r}_{a}}^{\phi_{i+1, k}^{a}} C_{i+1, k, g, \boldsymbol{r}_{a}}^{a_{z}}+R_{g \rightarrow g, \boldsymbol{r}_{b} \rightarrow \boldsymbol{r}_{a}}^{\phi_{i+1, k}^{a}} C_{i+1, k, g, \boldsymbol{r}_{b}}^{a_{z}}+R_{g \rightarrow g, a_{z} \rightarrow \boldsymbol{r}_{a}}^{\phi_{i+1, k}^{a}} C_{i+1, k, g,-a_{z}}^{a_{z}}+R_{g \rightarrow g,-a_{z} \rightarrow \boldsymbol{r}_{a}}^{\phi_{i+1, k}^{a}} C_{i+1, k, g,-}^{a_{z}}
\end{aligned}
$$

and

$$
G_{g, i+1, k, r_{a}}^{a_{z}}=R_{g \rightarrow g, r_{a} \rightarrow r_{a}}^{\phi_{i+1, k}^{a}} C_{i+1, k, g, r_{a}}^{a_{z}}+R_{g \rightarrow g, r_{b} \rightarrow r_{a}}^{\phi_{i+1, k}^{a}} C_{i+1, k, g, r_{b}}^{a_{z}}+R_{g \rightarrow g, a_{z} \rightarrow r_{a}}^{\phi_{i+1, k}^{a}} C_{i+1, k, g,-a_{z}}^{a_{z}}+R_{g \rightarrow g,-a_{z} \rightarrow r_{a}}^{\phi_{i+1, k}^{a}} C_{i+1, k, g,-a_{z}}^{a_{z}}
$$

The net currents on the node edges away from the interface between the two nodes are replaced with expressions obtained from the response balance formulation. The substitution into Equation (93) yields

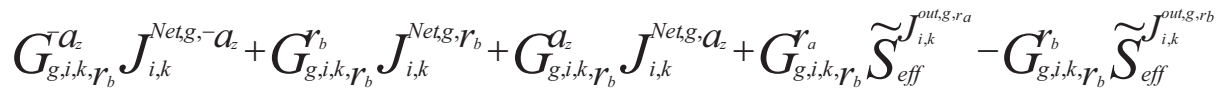

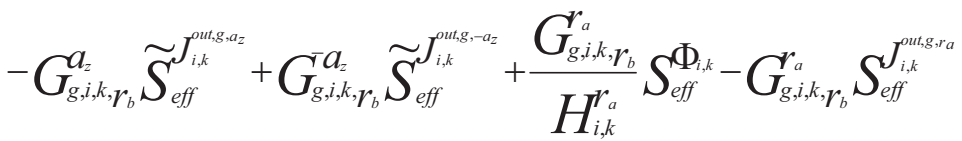

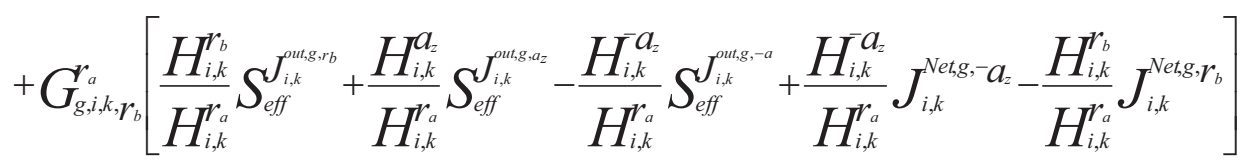

$$
\begin{aligned}
& -G_{g, i, k, r_{b}}^{r_{a}}\left[\frac{H_{i, k}^{a_{z}}}{H_{i, k}^{r_{a}}} J_{i, k}^{\mathrm{Netg}, a_{z}}+\frac{1}{H_{i, k}^{r}} \Phi_{i, k}\right] \\
& =G_{g, i+1, k, r_{a}}^{r_{a}} J_{i+1, k}^{\text {Netg, } r_{a}}+G_{g, i+1, k, r_{a}}^{a_{z}} J_{i+1, k}^{\text {Netg, } a_{z}}
\end{aligned}
$$

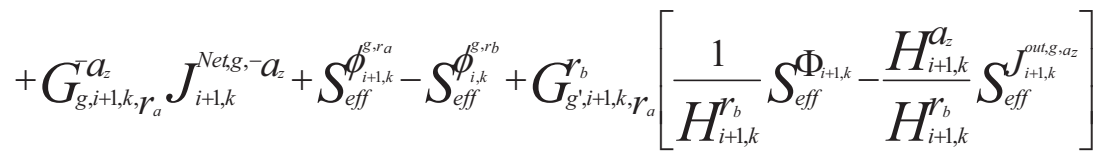

$$
\begin{aligned}
& +G_{g^{\prime}, i+1, k, r_{a}}^{r_{b}}\left[-\frac{H_{i+1, k}^{r_{a}}}{H_{i+1, k}^{r_{b}}} S_{e f f}^{J_{i+1, k}^{\text {out, r, ra }}}-\frac{H_{i+1, k}^{a_{z}}}{H_{i, k+1}^{r_{b}}} S_{e f f}^{J_{i+1, k}^{\text {out, }, a_{z}}}+\frac{H_{i+1, k}^{r_{b}}}{H_{i, k+1}^{r_{b}}} S_{e f f}^{J_{i+1, k}^{\text {out, r, }}}-\frac{H_{i+1, k}^{r_{a}}}{H_{i+1, k}^{r_{b}}} J_{i+1, k}^{\text {Netg, }, r_{a}}-\frac{H_{i+1, k}^{a_{z}}}{H_{i+1, k}^{r_{b}}} J_{i+1, k}^{\text {Netg. } a_{z}}\right] \\
& -G_{g^{\prime}, i+1, k, r_{a}}^{r_{b}}\left[\frac{H_{i+1, k}^{a_{z}}}{H_{i+1, k}^{r_{b}}} J_{i, k+1}^{\text {Net,g, } a_{z}}+\frac{1}{H_{i+1, k}^{r_{b}}} \Phi_{i+1, k}\right]+G_{g, i+1, k, r_{a}}^{r_{a}}{\widetilde{S_{e f f}}}_{J_{i+1, k}^{\text {out, }, r_{a}}}-G_{g, i+1, k, r_{a}}^{r_{b}} \widetilde{S}_{e f f}^{J_{i+1, k}^{\text {out, r, }}} \\
& -G_{g, i, k+1, r_{a}}^{a_{z}} \widetilde{S}_{e f f}^{J_{i, k+1, a_{z}}^{\text {out }}}+G_{g, i, k+1, r_{a}}^{a_{z}} \widetilde{S}_{e f f}^{J_{i, k+1}^{\text {out, }, a_{z}}}
\end{aligned}
$$


The net current at the outer side of the node (i,k) is the same net current at the inner side of the node $(\mathrm{i}+1, \mathrm{k})$. Collecting common interface net current and other like terms yields

$$
\begin{aligned}
& \bar{J}^{\text {Netg }}=\frac{G_{g, i, k, r_{b}}^{r_{a}}}{\tau H_{i, k}^{r_{a}}} \Phi_{i, k}-\frac{G_{g, i, k, r_{b}}^{r_{a}}}{\tau H_{i, k}^{r_{a}}} S_{e f f}^{\Phi_{i, k}}-\frac{1}{\tau}\left[G_{g, i, k, r_{b}}^{a_{z}}-\frac{H_{i, k}^{a_{z}}}{H_{i, k}^{r_{a}}} G_{g, i, k, r_{b}}^{r_{a}}\right] J_{i, k}^{\text {Netg, } a_{z}} \\
& -\frac{1}{\tau}\left[G_{g, i, k, r_{b}}^{a_{z}}-\frac{H_{i, k}^{a_{z}}}{H_{i, k}^{r_{a}}} G_{g, i, k, r_{b}}^{r_{a}}\right] J_{i, k}^{N e t, g,-a_{z}}-\frac{1}{\tau}\left[G_{g, i, k, r_{b}}^{r_{a}}-\frac{H_{i, k}^{a_{z}}}{H_{i, k}^{r_{a}}} G_{g, i, k, r_{b}}^{r_{a}}\right] S_{e f f}^{J_{i, k}^{\text {out, }, r_{a}}} \\
& -\frac{1}{\tau}\left[G_{g, i, k, r_{b}}^{-a_{z}}-\frac{H_{i, k}^{-a_{z}}}{H_{i, k}^{r_{a}}} G_{g, i, k, r_{b}}^{r_{a}}\right] S_{e f f}^{J_{i, k}^{o u, q, a a_{z}}}+\frac{1}{\tau}\left[G_{g, i, k, r_{b}}^{a_{z}}-\frac{H_{i, k}^{a_{z}}}{H_{i, k}^{r_{b}}} G_{g, i, k, r_{b}}^{r_{a}}\right] S_{e f f}^{J_{i, k}^{o u, g, a_{z}}} \\
& +\frac{1}{\tau}\left[G_{g, i+1, k, r_{a}}^{a_{z}}-\frac{H_{i+1, k}^{a_{z}}}{H_{i+1, k}^{r_{b}}} G_{g^{\prime}, i+1, k, r_{a}}^{r_{b}}\right] J_{i+1, k}^{\text {Net, },-a_{z}}+\frac{1}{\tau}\left[G_{g, i+1, k, r_{a}}^{a_{z}}-\frac{H_{i+1, k}^{a_{z}}}{H_{i+1, k}^{r_{b}}} G_{g^{\prime}, i+1, k, r_{a}}^{r_{b}}\right] J_{i+1, k}^{N e t, g, a_{z}} \\
& +\frac{1}{\tau}\left[G_{g, i+1, k, r_{a}}^{r_{a}}-\frac{H_{i+1, k}}{H_{i+1, k}^{r_{b}}} G_{g^{\prime}, i+1, k, r_{a}}^{r_{b}}\right] S_{e f f}^{J_{i, k+1}^{\text {out, ,ra }}}-\frac{1}{\tau}\left[G_{g, i+1, k, r_{a}}^{a_{z}}-\frac{H_{i+1, k}^{a_{z}}}{H_{i+1, k}^{r_{b}}} G_{g^{\prime}, i+1, k, r_{a}}^{r_{b}}\right] S_{e f f}^{J_{i+1, k}^{\text {out, , az }}} \\
& +\frac{1}{\tau}\left[G_{g, i+1, k, r_{a}}^{a_{z}}-\frac{H_{i+1, k}^{a_{z}}}{H_{i+1, k}^{r_{b}}} G_{g^{\prime}, i+1, k, r_{a}}^{r_{b}}\right] S_{e f f}^{J_{i+1, k}^{o u,-,-a_{z}}}-\frac{1}{\tau} \frac{G_{g^{\prime}, i+1, k, r_{a}}^{b_{b}}}{H_{i+1, k}^{r_{b}}} \Phi_{i, k+1}+\frac{1}{\tau} \frac{G_{g^{\prime}, i+1, k, r_{a}}^{r_{b}}}{H_{i+1, k}^{r_{b}}} S_{e f f}^{\Phi_{i+1, k}}
\end{aligned}
$$

where

$$
\tau=G_{g, i, k, r_{b}}^{r_{b}}-G_{g, i, k, r_{b}}^{r_{a}} \frac{H_{i, k}^{r_{b}}}{H_{i, k}^{r_{a}}}-G_{g, i+1, k, r_{a}}^{r_{a}}+G_{g, i+1, k, r_{a}}^{r_{b}} \frac{H_{i+1, k}^{r_{a}}}{H_{i+1, k}^{r_{b}}}
$$


Equation (103) is the expression for the net current at the boundary outer edge of a response treated node adjacent to another response treated node. A similar expression for the net current can be derived for the inner node edge, either by following the same derivation steps as above or by shifting the node index for the radial direction. The end result by either method yields

$$
\begin{aligned}
& \bar{J}^{\text {Net,g }}=\frac{G_{g, i, k, r_{a}}^{b}}{\tau H_{i, k}^{r_{b}}} \Phi_{i, k}-\frac{G_{g, i, k}^{b_{r}} r_{a}}{\tau H_{i, k}^{r_{b}}} S_{e f f}^{\Phi_{i, k}}-\frac{1}{\tau}\left[G_{g, i, k, r_{a}}^{a_{z}}-\frac{H_{i, k}^{a_{z}}}{H_{i, k}^{r_{a}}} G_{g, i, k, r_{b}}^{r_{a}}\right] J_{i, k}^{N e t, g, a_{z}} \\
& -\frac{1}{\tau}\left[G_{g, i, k, r_{a}}^{a_{z}}-\frac{H_{i, k}^{a_{z}}}{H_{i, k}^{r_{b}}} G_{g, i, k, r_{a}}^{r_{b}}\right] J_{i, k}^{N e t, g,-a_{z}}-\frac{1}{\tau}\left[G_{g, i, k, r_{a}}^{r_{a}}-\frac{H_{i, k}^{r_{a}}}{H_{i, k}^{r_{b}}} G_{g, i, k, r_{a}}^{r_{b}}\right] S_{e f f}^{J_{i, k, k, r_{a}}^{\text {ou }}}
\end{aligned}
$$

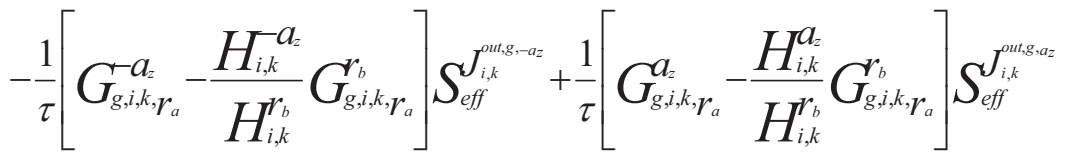

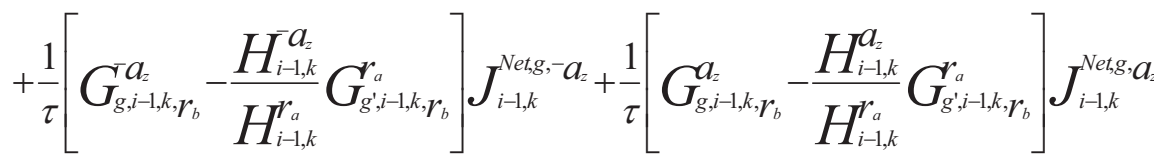

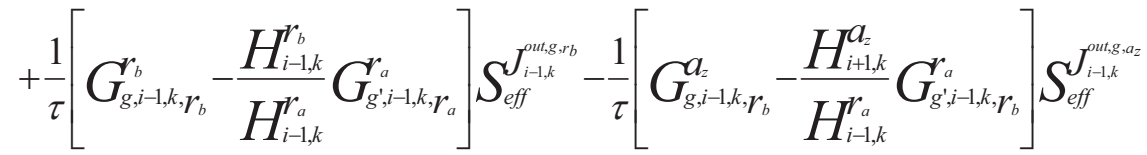

$$
\begin{aligned}
& +\frac{1}{\tau}\left[G_{g, i-1, k, r_{a}}^{a_{z}}-\frac{H_{i-1, k}^{a_{z}}}{H_{i-1, k}^{r_{b}}} G_{g^{\prime}, i-1, k, r_{a}}^{r_{b}}\right] S_{e f f}^{J_{i-1, k}^{o u, g,-a_{z}}}-\frac{1}{\tau} \frac{G_{g^{\prime}, i-1, k, r_{b}}^{r_{a}}}{H_{i-1, k}^{r_{a}}} \Phi_{i-1, k}+\frac{1}{\tau} \frac{G_{g^{\prime}, i-1, k, r_{b}}^{r_{a}}}{H_{i-1, k}^{r_{a}}} S_{e f f}^{\Phi_{i+1, k}}
\end{aligned}
$$

where

$$
\tau=G_{g, i, k, r_{a}}^{r_{a}}-G_{g, i, k, r_{a}}^{r_{b}} \frac{H_{i, k}^{r_{b}}}{H_{i, k}^{r_{a}}}-G_{g, i-1, k, r_{b}}^{r_{b}}+G_{g, i-1, k, r_{b}}^{r_{a}} \frac{H_{i-1, k}^{r_{a}}}{H_{i-1, k}^{r_{b}}}
$$

\subsection{Common Interface Current for a Response Node Adjoining a Diffusion Node on the Radial Boundary}

The expression for the interface net current of nodes is derived in this section. One of the nodes is identified as a diffusion node while the neighboring node is identified as a transport or response node. The diffusion node is treated using the Green's function solution method for the neutron diffusion equation while the response node is treated with a method based on response functions. The two nodes are assumed to be adjacent to each other in the radial direction.

To start the derivation for the equation of the interface net current between two nodes, a continuity/discontinuity condition of the scalar flux is stated at the interface boundary condition. A discontinuity condition of the interface scalar flux is needed since the scalar flux may be allowed to be discontinuous across the node boundaries of the diffusion treated nodes, in accordance with modern homogenization equivalence theory. The equations for the interface scalar fluxes from either side of the interface are substituted into the expression for the edge flux continuity/discontinuity equation that applies between the two adjacent nodes. The diffusion side scalar flux is expressed using the Green's function formulation within the context of diffusion theory while for the side of the response node the 
scalar flux is given as the response to incoming currents. The incoming partial currents for the response side are then replaced with expressions involving the net currents. This results in an equation that contains net currents and sources. The net currents for the opposite node edges, those away from the common interface, are then replaced with expressions obtained from the balance equation over each node. This substitution gives an expression that contains the common interface current, the average flux within each node, the transverse direction interface currents, and the effective sources of each node. After some algebraic manipulation, the common interface net current between a diffusion node and a response node is obtained.

A response and a diffusion node can be adjacent in the radial direction in two different ways: the response node may be either the innermost or the outermost of the two. In each situation, the flux at each nodal interface is allowed to be discontinuous, and thus a discontinuity condition is needed to relate the two fluxes at the interface. The discontinuity condition for a diffusion node to the outermost side of a response node is

$$
f_{i+1, k}^{l} \phi^{\text {diffusion }}=\phi^{\text {tr }}
$$

The factor multiplying the flux on the diffusion side is a discontinuity factor. The derivation and theory of these factors are well known and are not detailed here. The index $l$ given in Equation (105) stands for the left (or outermost) discontinuity factor. The continuity condition with a response node to the innermost side of a diffusion node is

$$
f_{i-1, k}^{r} \phi^{\text {diffusion }}=\phi^{t r}
$$

The index $r$ given in Equation (106) stands for the right (or innermost) discontinuity factor. Equations for the scalar flux at the common interface boundary are needed to obtain the common interface net current. Equations (88) and (89) are the expressions for the scalar flux at the radial boundaries of a response node. The Green's function expression for scalar flux at the left boundary of a diffusion node is

$$
\phi_{i, k}^{r_{a}}=r_{a} G\left(r_{a}, r_{a}\right) J_{i, k}^{N e t, g, r_{a}}-r_{b} G\left(r_{b}, r_{a}\right) J_{i, k}^{N e t, g, r_{b}}+\int_{r_{a}}^{r_{b}} r_{0} G\left(r_{a}, r_{0}\right) S_{0}\left(r_{0}\right) d r_{0}
$$

and the Green's function formulation of scalar flux for the right boundary of the diffusion node is

$$
\phi_{i, k}^{r_{b}}=r_{a} G\left(r_{a}, r_{b}\right) J_{i, k}^{N e t, g, r_{a}}-r_{b} G\left(r_{b}, r_{b}\right) J_{i, k}^{N e t, g, r_{b}}+\int_{r_{a}}^{r_{b}} r_{0} G\left(r_{b}, r_{0}\right) S_{0}\left(r_{0}\right) d r_{0}
$$

In Equations (107) and (108) the right side of the node boundary is denoted $\mathrm{r}_{\mathrm{b}}$ and the left side of the node boundary is denoted by $\mathrm{r}_{\mathrm{b}}$. The index $i$ is the radial node spatial index, and the index $k$ is the axial node spatial index. The index $g$ denotes the current energy group. The integral term is the source term for the diffusion treated node. The source term includes the transverse leakage term, the down-scatter, the upscatter, and the fission sources. The function multiplying the net boundary currents and the one integrated with the source terms is the radial Green's function. The derivation of the discretization of the diffusion equation and the nodal solution using Green's functions in the radial direction is similar to the same in the axial direction $^{3,4}$ and is not derived here.

Equation (105) expresses the continuity of the scalar flux at the boundary where a node treated with diffusion is to the right of a node treated with transport or response functions. The scalar flux term at the 
boundary on the diffusion side in Equation (105) is replaced by the scalar flux given in Equation (107); the scalar flux term at the boundary on the response node side given in Equation (105) is replaced by the scalar flux expressed in Equation (89). After substitutions, the resulting equation is

$$
\begin{aligned}
& f_{i+1, k}\left[r_{a, i+1, k} G\left(-r_{a, i+1, k},-r_{a, i+1, k}\right) J_{i+1, k}^{N e t g, r_{a}}-r_{b, i+1, k} G\left(-r_{a, i+1, k}, r_{b, i+1, k}\right) J_{i+1, k}^{N e t g, r_{b}}+\int_{r_{a, i+1, k}}^{r_{b, i+1, k}} r_{0} G\left(r_{a, i+1, k}, r_{0}\right) S_{0}\left(r_{0}\right) d r_{0}\right]
\end{aligned}
$$

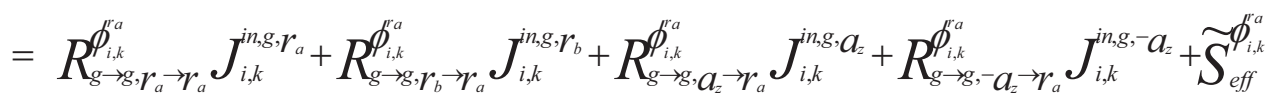

Equation (109) expresses the discontinuity condition of the scalar flux as a combination of net currents and the incoming partial currents. However, the sought equation is to involve net interface currents. Thus Equations (41), (42), (45), and (46) are substituted into Equation (109). The terms for the net currents and sources are grouped together, and the result is

$$
\begin{aligned}
& f_{i+1, k} r_{a, i+1, k} G\left(r_{a, i+1, k}, r_{a, i+1, k}\right) J_{i+1, k}^{N e t, g, r_{a}}-f_{i+1, k}^{l} r_{b, i+1, k} G\left(r_{a, i+1, k}, r_{b, i+1, k}\right) J_{i+1, k}^{N e t, g, r_{b}}+f_{i+1, k} \int_{r_{a, i+1, k}}^{r_{b, i+1, k}} r_{0} G\left(r_{a, i+1, k}, r_{0}\right) S_{0}\left(r_{0}\right) d r_{0} \\
& =G_{g, i, k, r_{b}}^{a_{a}} J_{i, k}^{N e t, g, r_{a}}+G_{g, i, k, r_{b}}^{r_{b}} J_{i, k}^{\text {Net,g, } r_{b}}+G_{g, i, k, r_{b}}^{a_{z}} J_{i, k}^{N e t, g, a_{z}}
\end{aligned}
$$

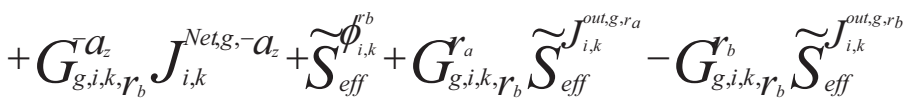

$$
\begin{aligned}
& -G_{g, i, k, r_{b}}^{a_{z}} \widetilde{S}_{e f f}^{J_{i, k}^{o u, g, a_{z}}}+G_{g, i, k, r_{b}}^{a_{z}} \widetilde{S}_{e f f}^{J_{i, k}^{\text {out, }, a_{z}}}
\end{aligned}
$$

The definition of the coefficients that multiply the net currents and the effective sources on the side of the response node are given Equations (94), (95), (96), and (97). Next, a set of expressions for the opposite face net currents are derived from the nodal balance equation and substituted into Equation (101). The right radial current for the diffusion node is replaced by an equation derived from the balance equation. However, the left radial current for the response node is replaced by an equation derived from Equation (89). The result is an equation that relates the common interface net current to the transverse net currents of the response node, the average scalar flux of both nodes, and effective sources of both nodes. The resulting equation from the substitution is 


$$
\begin{aligned}
& \left.f_{i+1, k} r_{a, i+1, k} G\left(r_{a, i+1, k}, r_{a, i+1, k}\right) J_{i+1, k}^{\mathrm{Netg}, r_{a}}-f_{i+1, k} G\left(r_{a, i+1, k}, r_{b, i+1, k}\right) \frac{2}{\left(r_{b, i+1, k}^{2}-r_{a, i+1, k}^{2}\right.}\right) \bar{S}_{0} \\
& +f_{i+1, k} G\left(r_{a, i+1, k}, r_{b, i+1, k}\right) \frac{2}{\left(r_{b, i+1, k}^{2}-r_{a, i+1, k}^{2}\right.} \Phi_{i+1, k}-f_{i+1, k}^{l} G\left(r_{a, i+1, k}, r_{b, i+1, k}\right) r_{a, i+1, k} J_{i+1, k}^{N e t g, r_{a}} \\
& +f_{i+1, k} \frac{2}{\left(r_{b, i+1, k}^{2}-r_{a, i+1, k}^{2}\right)} \int_{r_{a, i+1, k}}^{r_{b, i+1, k}} r_{0} G\left(r_{a, i, k+1}, r_{0}\right) S_{0}\left(r_{0}\right) d r_{0} \\
& =G_{g, i, k, r_{b}}^{-a_{2}} J_{i, k}^{\text {Netg, }-a_{z}}+G_{g, i, k, r_{b}}^{r_{b}} J_{i, k}^{\text {Netg, } r_{b}}
\end{aligned}
$$

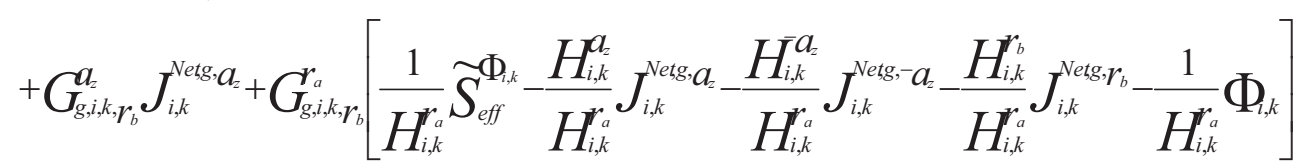

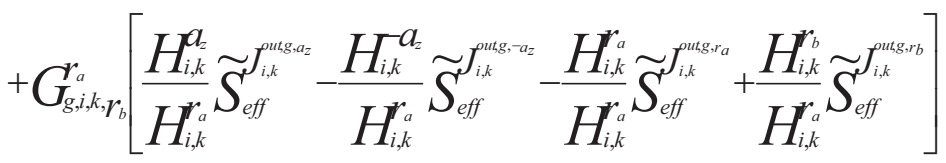

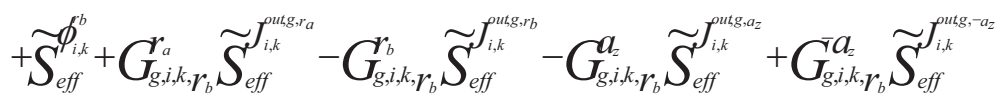

In Equation (111), the transverse leakage terms for the node with the diffusion treatment are incorporated into the source terms, but the transverse leakage net currents for the response node are expressed explicitly. An algebraic manipulation is next performed to obtain the common interface net current. The equation for the common interface current for a diffusion treated node to the right of a response treated node is

$$
\begin{aligned}
& J^{N e t}=-\frac{f_{i+1, k}^{l}}{\tau} \int_{r_{a, i+1, k}}^{r_{b, i+1, k}} r_{0} G\left(r_{a, i+1, k}, r_{0}\right) S_{0}\left(r_{0}\right) d r_{0}+\frac{2 f_{i+1, k}^{l}}{\left(r_{b, i+1, k}^{2}-r_{a, i+1, k}^{2}\right) \tau} G\left(r_{a, i+1, k}, r_{b, i+1, k}\right) \bar{S}_{0} \\
& +\frac{2 f_{i+1, k}^{l}}{\left(r_{b, i+1, k}^{2}-r_{a, i+1, k}^{2}\right) \tau} G\left(r_{b, i+1, k}, r_{a, i+1, k}\right) \Phi_{i+1, k}-\frac{1}{\tau} \frac{G_{g, i, k, r_{b}}^{r_{a}}}{H_{i, k}^{r_{a}}} \Phi_{i, k}+\frac{1}{\tau} \frac{G_{g, i, k, r_{b}}^{r_{a}}}{H_{i, k}^{r_{a}}} \widetilde{S}_{\text {eff }}^{\Phi_{i, k}} \\
& +\frac{1}{\tau}\left[G_{g, i, k, r_{b}}^{a_{z}}-\frac{H_{i, k}^{a_{z}}}{H_{i, k}^{r_{a}}} G_{g, i, k, r_{b}}^{r_{a}}\right] J_{i, k}^{N e t, g, a_{z}}+\frac{1}{\tau}\left[G_{g, i, k, r_{b}}^{-a_{z}}-\frac{H_{i, k}^{-a_{z}}}{H_{i, k}^{r_{a}}} G_{g, i, k, r_{b}}^{r_{a}}\right] J_{i, k}^{N e t, g,-a_{z}} \\
& -\frac{1}{\tau}\left[G_{g, i, k, r_{b}}^{a_{z}}-\frac{H_{i, k}^{a_{z}}}{H_{i, k}^{r_{a}}} G_{g, i, k, r_{b}}^{r_{a}}\right] \widetilde{S}_{e f f}^{J_{i, k}^{o u, g, a_{z}}}+\frac{1}{\tau}\left[G_{g, i, k, r_{b}}^{-a_{z}}-\frac{H_{i, k}^{-a_{z}}}{H_{i, k}^{r_{a}}} G_{g, i, k, r_{b}}^{r_{a}}\right] \widetilde{S}_{e f f}^{J_{i, k}^{\text {out, },-a_{z}}} \\
& -\frac{1}{\tau}\left[G_{g, i, k, r_{b}}^{r_{b}}-\frac{H_{i, k}^{r_{b}}}{H_{i, k}^{r_{a}}} G_{g, i, k, r_{b}}^{r_{a}}\right] \widetilde{S}_{e f f}^{J_{i, k}^{\text {out, },, r_{b}}}+\frac{1}{\tau} \widetilde{S}_{e f f}^{\phi_{i, k}^{r_{b}}}
\end{aligned}
$$

where

$$
\tau=f_{i+1, k}^{l} r_{a, i+1, k} G\left(r_{a, i+1, k}, r_{a, i+1, k}\right)-f_{i+1, k}^{l} r_{b, i+1, k} G\left(r_{b, i+1, k}, r_{a, i+1, k}\right)-G_{g, i, k, r_{b}}^{r_{b}}+\frac{H_{i, k}^{r_{b}}}{H_{i, k}^{r_{a}}} G_{g, i, k, r_{b}}^{r_{a}}
$$


Equation (112) is an expression for the net current at the interface between a node treated with diffusion and a node treated with response functions. Equation (112) relates the interface net current of the two adjacent nodes to the average fluxes of each node and the sources, which include the transverse leakage terms.

A similar equation can be derived for the interface net current when the node treated with response functions is to the right of a node treated with diffusion theory and the Green's function solution approach. The derivation of the equation for the interface net current in this latter case is similar to the derivation presented above for the net current when the response node is to the left of a diffusion node. The equation for the net current at the interface with a diffusion-treated node is to the left of a response function treated node is

$$
\begin{aligned}
& J^{N e t}=-\frac{f_{i-1, k}^{r}}{\tau} \int_{r_{a, i-1, k}}^{r_{b, i-1, k}} r_{0} G\left(r_{b, i-1, k}, r_{0}\right) S_{0}\left(r_{0}\right) d r_{0}+\frac{2 f_{i-1, k}^{r}}{\left(r_{b, i-1, k}^{2}-r_{a, i-1, k}^{2}\right) \tau} G\left(r_{a, i-1, k}, r_{b, i-1, k}\right) \bar{S}_{0} \\
& +\frac{2 f_{i-1, k}^{r}}{\left(r_{b, i-1, k}^{2}-r_{a, i-1, k}^{2}\right) \tau} G\left(r_{a, i-1, k}, r_{b, i-1, k}\right) \Phi_{i-1, k}-\frac{1}{\tau} \frac{G_{g, i, k, r_{a}}^{r_{b}}}{H_{i, k}^{r_{b}}} \Phi_{i, k}+\frac{1}{\tau} \frac{G_{g, i, k, r_{a}}^{r_{b}}}{H_{i, k}^{r_{b}}} \widetilde{S}_{e f f} \Phi_{i, k} \\
& +\frac{1}{\tau}\left[G_{g, i, k, r_{a}}^{-a_{z}}-\frac{H_{i, k}^{-a_{z}}}{H_{i, k}^{r_{b}}} G_{g, i, k, r_{a}}^{r_{b}}\right] J_{i, k}^{\text {Net, },-a_{z}}+\frac{1}{\tau}\left[G_{g, i, k, r_{a}}^{a_{z}}-\frac{H_{i, k}^{a_{z}}}{H_{i, k}^{r_{b}}} G_{g, i, k, r_{a}}^{r_{b}}\right] J_{i, k}^{\text {Net, }, a_{z}} \\
& +\frac{1}{\tau}\left[G_{g, i, k, r_{a}}^{-a_{z}}-\frac{H_{i, k}^{-a_{z}}}{H_{i, k}^{r_{a}}} G_{g, i, k, \boldsymbol{r}_{a}}^{r_{b}}\right] \widetilde{S}_{\text {eff }}^{J_{i, k}^{\text {out, }, a_{z}}}-\frac{1}{\tau}\left[G_{g, i, k, \boldsymbol{r}_{a}}^{a_{z}}-\frac{H_{i, k}^{a_{z}}}{H_{i, k}^{r_{b}}} G_{g, i, k, \boldsymbol{r}_{a}}^{r_{b}}\right] \widetilde{S}_{\text {eff }}^{J_{i, k}^{\text {out, }, a_{z}}} \\
& +\frac{1}{\tau}\left[G_{g, i, k, r_{a}}^{r_{a}}-\frac{H_{i, k}^{r_{a}}}{H_{i, k}^{r_{b}}} G_{g, i, k, r_{a}}^{r_{b}}\right] \widetilde{S}_{e f f}^{J_{i, k}^{o u, g, r b}}+\frac{1}{\tau} \widetilde{S}_{e f f}^{\phi_{i, k}^{r a}}
\end{aligned}
$$

where

$$
\tau=f_{i-1, k}^{r} r_{b, i-1, k} G\left(r_{b, i-1, k}, r_{b, i-1, k}\right)-f_{i-1, k}^{r} r_{b, i-1, k} G\left(r_{a, i-1, k}, r_{b, i-1, k}\right)-G_{g, i, k, r_{a}}^{r_{a}}+\frac{H_{i, k}^{r_{a}}}{H_{i, k}^{r_{b}}} G_{g, i, k, \boldsymbol{r}_{a}}^{r_{b}}
$$

\subsection{Common Interface Current for a Response Node Adjoining a Radial Boundary}

The net current for a response node at the boundary of a reactor is derived in this section. Only the last radial node is considered for the response function treatment. While the inner most node could be treated with response functions, they do not possess a significant practical interest in the investigation and modeling of control rods for pebble bed reactors.

The notation for boundary condition is established prior to carrying out the derivation. Then, the flux expression given by the boundary condition is matched to a response function expression for the boundary scalar flux. The result is an equation that contains incoming currents, sources, and net currents. The incoming currents are replaced by equations containing net currents and effective sources. The result of the substitution is an equation that involves the incoming current as a source, the net currents, and effective sources. The net current at the node inner edge of the node (the one opposite the outside 
boundary of the reactor) is replaced by an equation that contains average fluxes, transverse net currents, the reactor boundary net current, and effective sources for the node. This elimination of the opposite net current gives an expression that is then solved to obtain the net current along the reactor boundary.

The equation for the radial boundary condition of the reactor is

$$
J^{i n, r_{b}}=\alpha_{r_{b}} \phi^{r_{b}}+\beta_{r_{b}} J_{g}^{N e t, r_{b}}
$$

Equation (114) gives the incoming partial current in terms of the scalar flux and the net current evaluated at the reactor boundary. The coefficients, $\alpha_{\mathrm{rb}}, \beta_{\mathrm{rb}}$ and the incoming currents are part of the specified input conditions. For a boundary condition assuming linear anisotropy, the coefficients $\alpha_{\mathrm{rb}}$ is set equal to 0.25 , and the coefficient $\beta_{\mathrm{rb}}$ is set equal to -0.5 .

The next step in the derivation of the desired expression is the elimination of the surface flux between Equations (114) and (89). This gives

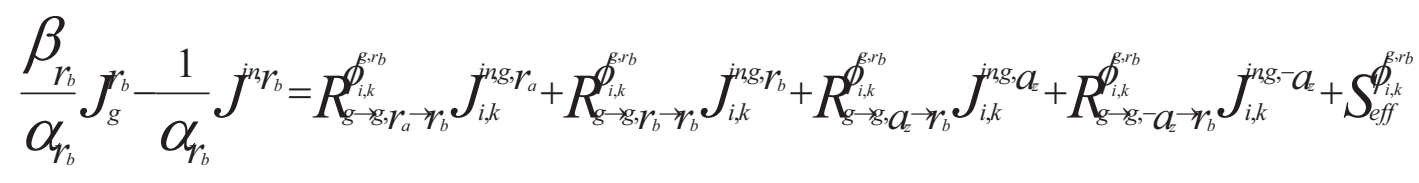

Equations (41), (42), (45), and (46) are introduced into Equation (115), replacing the four partial currents that appear multiplied by scalar flux response functions. Although the incoming partial current for the surface at $r_{b}$ that appears on the right side of Equation (115) may be specified as part of the boundary condition, the incoming current on side $r_{b}$ is replaced by its expression from the relevant equation, as just stated. The resulting equation is

$$
\begin{aligned}
& \frac{\beta_{r_{b}}}{\alpha_{r_{b}}} J_{g}^{N e t, g, r_{b}}-\frac{1}{\alpha_{r_{b}}} J^{i n, r_{b}} \\
& =G_{g, i, k, r_{b}}^{r_{a}} J_{i, k}^{\text {Net, }, r_{a}}+G_{g, i, k, r_{b}}^{r_{b}} J_{i, k}^{\text {Net,g, } r_{b}}+G_{g, i, k, r_{b}}^{a_{z}} J_{i, k}^{\text {Net, }, a_{z}}+G_{g, i, k, r_{b}}^{a_{z}} J_{i, k}^{\text {Net,g,- } a_{z}}
\end{aligned}
$$

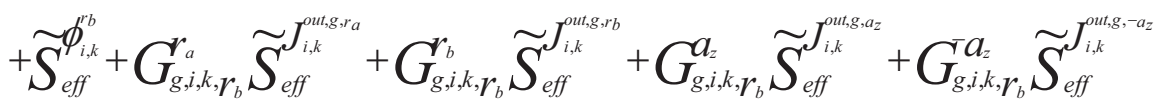


The coefficients in front of the net currents on the right side of Equation (116) are given by Equations (94), (95), (96), and (97). Next, the expression for the net current at the interface $r_{a}$, derived from the balance equation, is substituted into Equation (116). The result is

$$
\begin{aligned}
& \frac{\beta_{r_{b}}}{\alpha_{r_{b}}} J_{g}^{N e t, g, r_{b}}-\frac{1}{\alpha_{r_{b}}} J^{i n, r_{b}}
\end{aligned}
$$

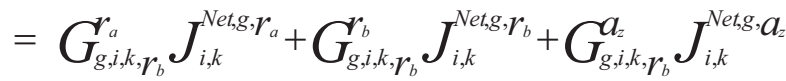

$$
\begin{aligned}
& +G_{g, i, k, r_{b}}^{a_{z}}\left[-\frac{H_{i, k}^{g,-} a_{z}}{H_{i, k}^{g, r_{a}}} J_{i, k}^{N e t, g,-a_{z}}-\frac{H_{i, k}^{g, r_{b}}}{H_{i, k}^{g, r_{a}}} J_{i, k}^{N e t, g, r_{b}}-\frac{H_{i, k}^{g, a_{z}}}{H_{i, k}^{g, r_{a}}} J_{i, k}^{N e t, g, a_{z}}-\frac{1}{H_{i, k}^{g, r_{a}}} \Phi^{g}\right]
\end{aligned}
$$

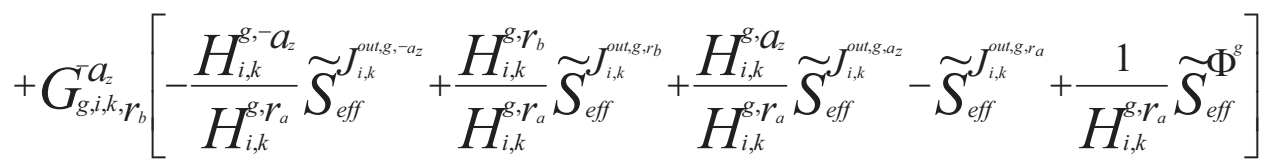

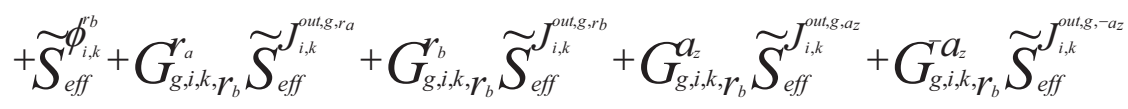

In Equation (117), terms for the net currents and effective sources are collected into separate groups. The net current on the reactor boundary is then obtained and is given by

$$
\begin{aligned}
& J^{N e t}=\frac{1}{\tau} \frac{1}{\alpha_{r_{b}}} J^{i n, a_{z}}-\frac{1}{\tau} \frac{G_{g, i, k, r_{b}}^{r_{a}}}{H_{i, k}^{r_{a}}} \Phi_{i, k}+\frac{1}{\tau} \frac{G_{g, i, k, r_{b}}^{r_{a}}}{H_{i, k}^{r_{a}}} \widetilde{S}_{\text {eff }}^{\Phi_{i, k}} \\
& +\frac{1}{\tau}\left[G_{g, i, k, r_{b}}^{a_{z}}-\frac{H_{i, k}^{a_{z}}}{H_{i, k}^{r_{a}}} G_{g, i, k, r_{b}}^{r_{a}}\right] J_{i, k}^{N e t, g, a_{z}}+\frac{1}{\tau}\left[G_{g, i, k, r_{b}}^{-a_{z}}-\frac{H_{i, k}^{-a_{z}}}{H_{i, k}^{r_{a}}} G_{g, i, k, r_{b}}^{r_{a}}\right] J_{i, k}^{N e t, g,-a_{z}} \\
& +\frac{1}{\tau}\left[G_{g, i, k, r_{b}}^{-a_{z}}-\frac{H_{i, k}^{-a_{z}}}{H_{i, k}^{r_{a}}} G_{g, i, k, r_{b}}^{r_{a}}\right] \widetilde{S}_{\text {eff }}^{J_{i t, k}^{\text {out, }, a_{z}}}-\frac{1}{\tau}\left[G_{g, i, k, r_{b}}^{r_{b}}-\frac{H_{i, k}^{r_{b}}}{H_{i, k}^{r_{a}}} G_{g, i, k, r_{b}}^{r_{a}}\right] \widetilde{S}_{\text {eff }}^{J_{i, k}^{\text {out, },, r b}} \\
& -\frac{1}{\tau}\left[G_{g, i, k, r_{b}}^{a_{z}}-\frac{H_{i, k}^{a_{z}}}{H_{i, k}^{r_{a}}} G_{g, i, k, r_{b}}^{r_{a}}\right] \widetilde{S}_{e f f}^{J_{i, k}^{o u, g, a_{z}}}+\frac{1}{\tau} \widetilde{S}_{e f f}^{\phi_{i, k}^{r_{b}}}
\end{aligned}
$$

where

$$
\tau=\frac{\beta_{r_{b}}}{\alpha_{r_{b}}}-G_{g, i, k, r_{b}}^{r_{a}}+\frac{H_{i, k}^{r_{b}}}{H_{i, k}^{r_{a}}} G_{g, i, k, r_{b}}^{r_{a}}
$$




\section{EMBEDDING THE RESPONSE FUNCTION NODES INTO THE MATRIX FOR THE SCALAR FLUX}

The formulation of the matrix for the scalar flux with the embedded response function methodology is discussed in this section. The solution strategy of the matrix and the calculation of the critical eigenvalue remain the same, and are documented elsewhere. ${ }^{5,6}$ To form the three- or five-striped matrix for the nodeaveraged scalar fluxes, the equations for the net currents at the node edges are derived. The edge net currents for nodes that are treated with response functions as well as through the diffusion method were derived in Sections 4 and 5.

The derivation of the node-edge net currents for nodes that are treated with diffusion theory and have diffusion treated neighbors can be found in well documented sources. ${ }^{5,7}$ The expressions for the nodeedge net currents and the nodal balance equations are manipulated to form the rows of the matrix equation for the nodal scalar fluxes. This is done by substituting the equations for the node-edge net currents into the appropriate balance equation. The result is an equation that contains the node average fluxes of the adjacent nodes, the source terms of the adjacent nodes, and the transverse leakages. The transverse leakages and source terms are moved to the right side of the equation and are treated as an effective source term.

In the implementation of the hybrid method within the CYNOD code, the matrix for the flux equation must be formed. The first step in this process, for each row, is to consult a special (input) array that specifies if the coupling coefficients of the node under consideration are to be obtained from the ordinary diffusion parameters or from response functions. If response functions are to be used, the array also specifies the type of the adjacent nodes. With this information, the matrix row entries are calculated by multiplying the coefficients in front of the net current terms in the appropriate neutron balance equation by the coefficients in front of the node average scalar fluxes given by the net current expressions obtained in Sections 4 and 5 and in References [5] and [6]. The node types information for the node under consideration and its neighbors is also used to construct the right side vector. The coefficients in front of the transverse net currents of the balance equation are multiplied by the transverse leakage and source terms related coefficients of the equations for the net currents derived in Sections 4 and 5. These terms are then moved to the right side. With the all the matrix coefficients and right sides determined, the resulting linear system of equations can be solved using established means. ${ }^{5}$

In the procedure just described for constructing the matrix equation, when a three-stripe system is built, the net currents of the transverse direction are incorporated into the source terms for the node. The equations in the axial direction then take the form

$$
A_{i, k+1}^{g, T} \Phi_{i, k+1}^{g}+\left(A_{i, k+1}^{g, B}+A_{i, k-1}^{g, T}\right) \Phi_{i, k}^{g}+A_{i, k-1}^{g, B} \Phi_{i, k-1}^{g}=Q_{i}^{g}
$$

The coefficients $A_{i, k+1}^{g, T}$ and $A_{i, k+1}^{g, B}$ result from the substitution of the top net current equation, and the collection of terms for the node-averaged scalar flux terms. The coefficients $A_{i, k-1}^{g, T}$ and $A_{i, k-1}^{g, B}$ result from the substitution of the bottom net current equation, and the collection of terms for thee node-averaged scalar flux terms. The form of these equations can differ depending on whether the coupling coefficients of the neighbor are obtained from response functions or diffusion parameters. The source $Q_{i}^{g}$ contains the up-scattering terms, the fission source terms, the down-scattering source terms, the transverse leakages, and any effective source terms. The transverse leakages are composed of the net currents in the radial direction. An equation similar to Equation (119) can be written for the radial direction. The axial net currents in this latter (radial) equation are incorporated into the effective source. 
Given the matrix formulation of Equation (119), the average nodal fluxes are obtained through the use of ordinary linear solvers. However, the solution strategy depends on whether the formulation adopted is three or five stripes. In the case of the five-stripe formulation, the solution is entirely a classical direct inversion or iterative inversion. In the case of the three-stripe formulation, the solution approach requires directional iterations between the $\mathrm{z}$ and $\mathrm{r}$-direction equations, with coupling taking place within the transverse leakage terms. The solution for a given directional can then be either through direct inversion or through iterations for each 1-D block matrix. The current implementation of the hybrid method supports only the three-stripe form with the requirement of directional iterations. 


\section{COMPUTATIONAL TESTS AND VERIFICATION OF METHOD IMPLEMENTATION}

The newly developed hybrid method described in the previous sections has been tested and verified. This section presents the approach used for testing and verification and shows the related results.

The ultimate testing and verification of the new method will require the availability of response functions derived using transport theory solvers. The development of such transport-theory-based response functions is beyond the scope of the NGNP Project this fiscal year. Instead, the project planned to leverage a US DOE NERI project at the Georgia Institute of Technology (GA-Tech) that is developing response functions based on the MCNP Monte Carlo code. The development of the response functions by GA-Tech is expected to be completed next fiscal year. Therefore, alternative response functions had to be devised to test the new hybrid methodology and the code that implements it,. This alternative to transporttheory-based response functions has been developed and coded, thus going beyond the initial plans for this project. The alternative response functions are based on diffusion theory and are formulated using fine mesh Green's function solutions. Diffusion-theory-based Green's function-based response functions have been derived and implemented into auxiliary codes then used to verify the new hybrid code.

To verify the implementation of the embedded response function treatment with discontinuity factors, the $r$ and $z$ directions were initially decoupled and each direction was tested separately. For testing the $\mathrm{z}$ direction implementation, a 1-D version of the PBMR benchmark problem was devised ${ }^{7}$ and used. The decoupled $\mathrm{z}$ direction implementation within the CYNOD code, created in this project, produced the same effective multiplication factor eigenvalue $\left(k_{e f f}\right)$ as that reported in Reference 7 . The decoupled $r$ direction was also tested. Discontinuity factors were obtained from a finite difference fine mesh calculation in the radial direction. The response functions were obtained by reformulating the radial Green's function solution of the CYNOD and PEBBED codes in terms of partial incoming and outgoing currents. The response functions were then coded into a MATLAB script and the resulting code was used to generate the needed data. The coarse mesh finite difference (CMFD) nodal r-direction code with the embedded response functions showed good agreement with the fine mesh finite difference solution.

A number of problems were generated and tested for the coupled r-z code; the test results from three of these problems are shown here. The first sample problem is a fictitious reactor $10 \mathrm{~m}$ high with a $10 \mathrm{~m}$ radius. The model discretization assumes five nodes in each direction. A fine mesh diffusion solution was generated in each case, from which discontinuity factors were computed. Response functions were generated by analytically rearranging the Nodal Green's function solution for the diffusion equation in terms of partial currents. The Green's function solutions were then used to compute numerical values for the response functions using the data shown in Table 1.Table 1 The same data are used for all three sample problems presented here.

Table 1. Diffusion theory data for test problems 1,2, and 3.

\begin{tabular}{|c|c|c|c|c|}
\hline & $\mathbf{D}(\mathbf{c m})$ & $\Sigma_{\mathbf{a}}\left(\mathbf{c m}^{-1}\right)$ & $\Sigma_{\mathbf{f}}\left(\mathbf{c m}^{-1}\right)$ & $v \Sigma_{\mathbf{f}}\left(\mathbf{c m}^{-1}\right)$ \\
\hline Material 1 (Blue) & 0.01 & 0.001 & 0.003 & 0.006 \\
\hline Material 2 (Red) & 0.01 & 0.1 & 0.0 & 0.0 \\
\hline
\end{tabular}

The configuration of the first test problem is shown in Figure 1. In this sample reactor problem a curtain (azimuthally symmetric) control rod is inserted down to two-fifths from the top. The red region represents the partially inserted control rod while the rest of the reactor, shaded in blue, is the fuel region. The coarse mesh node spacing for this reactor is assumed constant. A single energy group is assumed and the cross sections and diffusion coefficient are the ones listed in Table 1. 


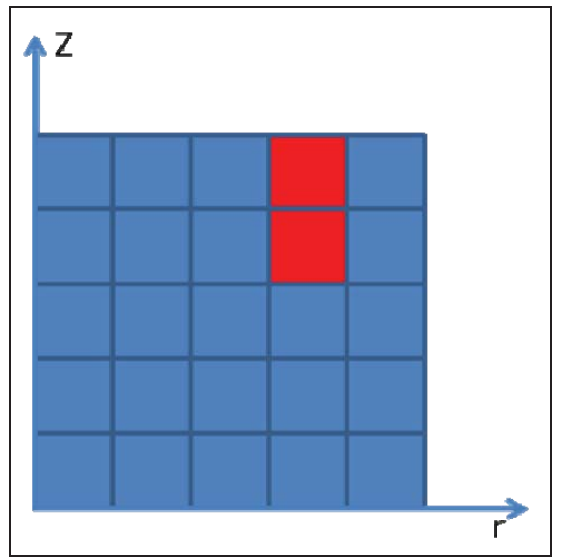

Figure 1. Test model with partially inserted control rods in top portion of reactor.

A reference solution for the sample problem is obtained using the Green's function nodal solution approach and refining the mesh simultaneously in both the $\mathrm{r}$ and $\mathrm{z}$ directions. The spatial convergence of $k_{\text {eff }}$ for the sample problem is shown in Figure 2 where the number of mesh cells shown on the abscissa is the same for each of the two directions. For example, 10 mesh cells means a $10 \times 10$ solution in the figure, which shows that the effective multiplication factor asymptotically approaches 1.0009 as the mesh is refined. From the figure it can also be seen that $40 \times 40$ is sufficiently spatially converged to be nearly indistinguishable from the asymptotic value reached with the $80 \times 80$ mesh. Therefore, the test discontinuity factors are computed from the $40 \times 40$ solution.

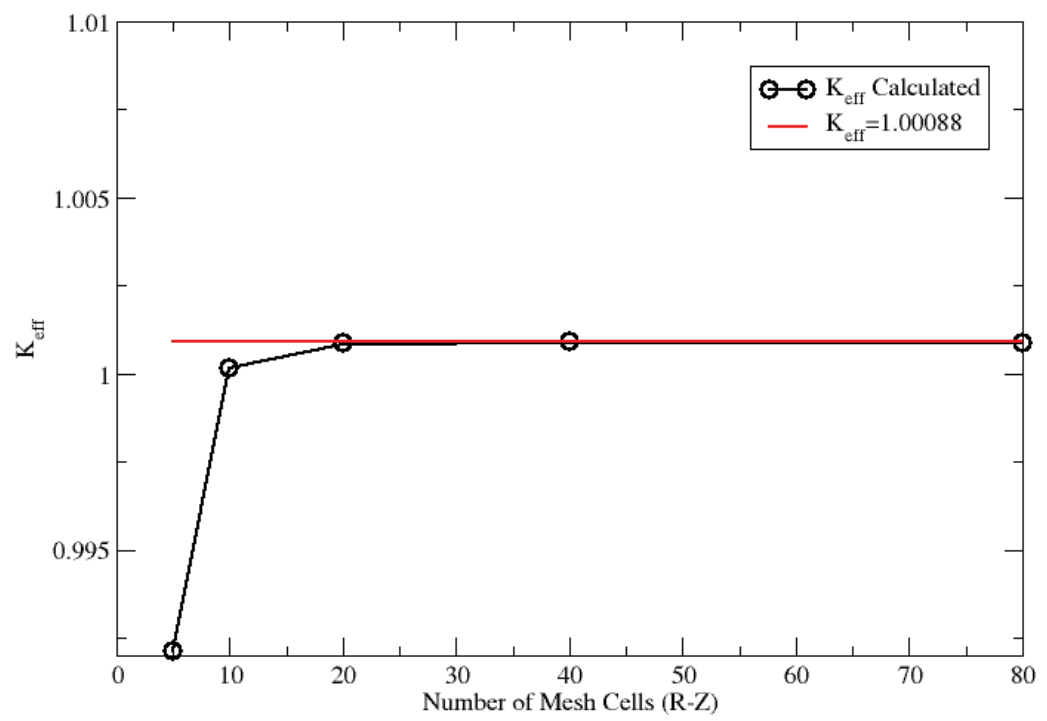

Figure 2. Spatial convergence and reference $\mathrm{k}_{\text {eff }}$ solution.

The testing of the new hybrid method is carried out through the use of the Green's function nodal solution approach in all nodes but the red ones, in which the response formulation is used. The results of the testing are shown in Figure 2. The four cases shown in the figure are the "Fine Mesh" reference solution, the "Pure Diffusion" solution obtained using the Green's function method throughout the domain, the "Diffusion with DF," which is the same as Pure Diffusion, but with discontinuity factors incorporated, and the Hybrid with DF, which uses Green's function method with discontinuity factors in all the fuel nodes and response functions (without discontinuity factors) in the controlled nodes. 
Table 2. Testing matrix for the hybrid method (partially inserted control rods from top).

\begin{tabular}{|l|c|c|}
\hline \multicolumn{3}{|c|}{ Partial Inserted Control Rod from above } \\
\hline \multicolumn{1}{|c|}{ Case } & Mesh spacing & Calculated $\boldsymbol{k}_{\text {eff }}$ \\
\hline Fine mesh & $(40 \times 40)$ & 1.00089 \\
\hline Pure Diffusion & $(5 \times 5)$ & 0.99212 \\
\hline Diffusion with DF & $(5 \times 5)$ & 1.00089 \\
\hline Hybrid with DF & $(5 \times 5)$ & 0.99817 \\
\hline
\end{tabular}

An examination of the results in Table 2 show that the $5 \times 5$ Diffusion with DF solution reproduces the reference solution exactly, which improves over the pure diffusion without discontinuity factors. In contrast, the Hybrid with DF does not reproduce the reference solution. Instead, as expected, its result is intermediate between the Diffusion with DF and the Pure Diffusion because the Hybrid uses DFs in all fuel nodes but not in the response nodes, which, although using response functions, are actually treated with diffusion theory in the test problem.

The configuration of the second test problem is shown in Figure 3. The configuration is symmetric to the previous case with top and bottom interchanged. The data are identical to those of the previous case. As expected, the results for this case are identical to those of the previous case, to the last digit as shown in Table 3. These results verify that the coding of the response function approach for top and bottom nodes, though implemented separately, is correct.

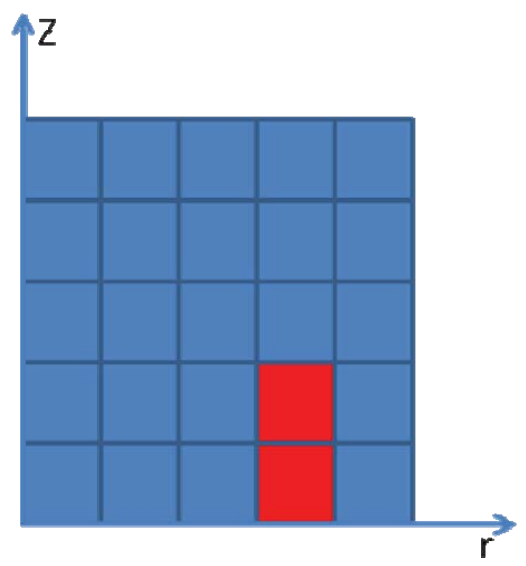

Figure 3 Test model with partially inserted control rods in bottom portion of reactor.

Table 3. Testing matrix for the hybrid method (partially inserted control rods from bottom).

\begin{tabular}{|l|c|c|}
\hline \multicolumn{3}{|c|}{ Partial Inserted Control Rod from below the core } \\
\hline Case & Mesh spacing & Calculated $\boldsymbol{k}_{\text {eff }}$ \\
\hline Fine mesh & $(40 \times 40)$ & 1.00089 \\
\hline Pure Diffusion & $(5 \times 5)$ & 0.99212 \\
\hline Diffusion with DF & $(5 \times 5)$ & 1.00089 \\
\hline Hybrid with DF & $(5 \times 5)$ & 0.99817 \\
\hline
\end{tabular}


The next example is a case where the control rod is fully inserted into the reactor. The dimensions for the reactor are the same as in the partial inserted control cases but the control rod is fully inserted as shown in Figure 4.

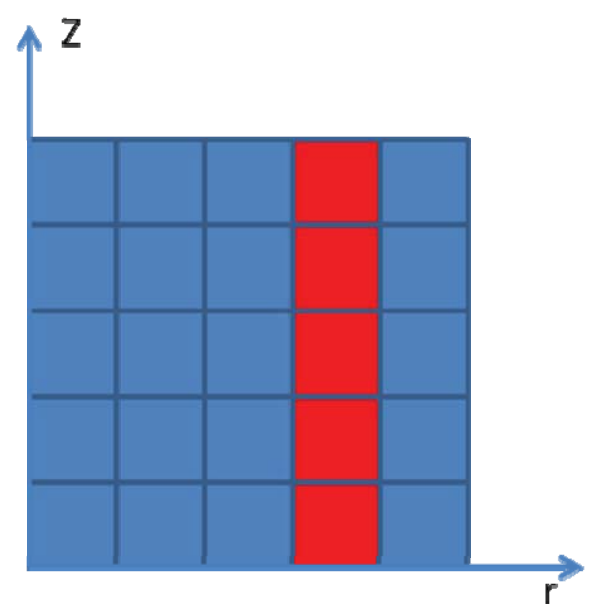

Figure 4. Test model with fully inserted curtain control rods.

This fully shutdown case is used to test the multigroup implementation by using two energy groups. This model problem was used with varying parameters to help test and debug the implementation. For the hybrid method, the red nodes are treated with the embedded response function formulation. The data used are given in Table 4.

Table 4. Diffusion theory data for test problem 4.

\begin{tabular}{|c|c|c|c|c|}
\hline Material 1 (Blue) & $\mathbf{D}(\mathbf{c m})$ & $\Sigma_{\mathbf{a}}\left(\mathbf{c m}^{-\mathbf{1}}\right)$ & $\Sigma_{\mathbf{f}}\left(\mathbf{c m}^{-\mathbf{1}}\right)$ & $\Sigma_{\mathbf{s 1}-\mathbf{2}}\left(\mathbf{c m}^{-\mathbf{1}}\right)$ \\
\hline Group 1 & 0.1 & 1.0 & 0.0 & 1.0 \\
\hline Group 2 & 1.0 & 0.1 & 1.0 & 0.0 \\
\hline Material 2 (Red) & & & & \\
\hline Group 1 & 1.0 & 0.1 & 0.0 & 0.1 \\
\hline Group 2 & 0.1 & 2.0 & 0.0 & 0.0 \\
\hline
\end{tabular}

The results for the fully shutdown reactor case are shown in Table 5.

Table 5. Testing matrix for the hybrid method (fully inserted control rods).

\begin{tabular}{|l|c|c|}
\hline \multicolumn{1}{|c|}{ Case } & Mesh spacing & Calculated $\boldsymbol{k}_{\text {eff }}$ \\
\hline Fine Mesh & $(20 \times 20)$ & 0.52919 \\
\hline Pure Diffusion & $(5 \times 5)$ & 0.50475 \\
\hline Diffusion with DF & $(5 \times 5)$ & 0.52671 \\
\hline Hybrid with DF & $(5 \times 5)$ & 0.52711 \\
\hline
\end{tabular}

The full shutdown case results are consistent, which demonstrates that the implementation of the hybrid method was carried out correctly. Another finding is that the use of discontinuity factors based on fine mesh diffusion calculation improves the results substantially. 
The last test that was performed was the computation of the effective multiplication factor for the OECD PBMR-400 Benchmark. ${ }^{8}$ This benchmark is defined without discontinuity factors, and thus none are used in the testing. Also, at the time of the writing of this report no very-fine mesh reference solution was available. Response functions based on transport theory are not available either. Therefore, the test is merely a comparison of the results obtained using the unmodified version of the CYNOD code with the results obtained using the modified version, which incorporates the response functions approach in the control nodes. In this latter version, the hybrid method, all the nodes that were identified as including control rods were replaced with response nodes. The two versions gave the exact same value of 1.00349 for the $k_{\text {eff }}$. This is agreement to six digits between the two methods.

Since the response function data were generated using the nodal Green's function solution, this level of agreement is expected. Indeed, the response functions should produce exactly the same result as the nodal Green's function solution implemented within the CYNOD code.

The four tests described in this section verify the implementation of the hybrid method. In particular, they demonstrate that the new portion of the code, i.e., the one pertaining to the implementation of the response function approach, has been coded correctly. This verification of the implementation provides confidence that the correct equations and models have been incorporated into the code. However, this verification does not constitute validation of the methodology. Validation must be carried out with comparisons against experiments or results from validated codes. 


\section{STATUS OF RADIAL FLUX SHAPE CORRECTION TASK}

In addition to the developments described in the previous sections and that pertain to the treatment of non-multiplying zones, a method for increasing the fidelity and accuracy of the nodal flux shape in the $r$ direction is under development. In its current state, the CYNOD (or PEBBED) code uses a flat flux approximation for the radial direction in the estimation of the effective sources that are used in the Green's function solution. This approximation is not inherent in the Green's function solution. The purpose of this task is the removal of the flat flux approximation in the source estimation. This is done by taking the analytical solution for the radial flux and expanding it into a (truncated) series of orthogonal polynomials using the expansion in the estimation of the source terms. This development was initiated in the course of a NERI project now terminated. However, the development of the expansions could not be completed in that project because of the shear magnitude of the task. This task is, therefore, resumed in this project and significant progress has been made towards completing the development. In the next several sections the description of the expansion methodology is given, followed by a presentation of the current status of the work.

\subsection{Solution for the Transverse-Integrated Radial Nodal Flux}

The solution of the 1-D radial direction transverse integrated nodal diffusion equation is (with usual notations)

$$
{ }^{z, \theta} \overline{\phi_{l}}(r)=\int_{a_{l}}^{b_{l}} G_{l}\left(r, r_{0}\right)^{z, \theta} \overline{S_{l}}\left(r_{0}\right) r_{0} d r_{0}+a_{l} G_{l}\left(r, a_{l}\right)^{z, \theta} \overline{J_{r, l}}\left(a_{l}\right)-b_{l} G_{l}\left(r, b_{l}\right)^{z, \theta} \overline{J_{r, l}}\left(b_{l}\right) .
$$

In order to be able to implement this solution numerically, the effective source term appearing in the integral must be expressed numerically. The most efficient and convenient way to do so is to approximate the effective source (and hence the underlying flux) through a polynomial expansion, the coefficients of which are repeatedly updated as the iterative process converges. The mathematical derivations needed for this purpose are being developed and the status of the development is presented in this report. The first step in this development is the selection of a suitable polynomial base. Such a base is described in the next section.

\subsection{Orthonormal Polynomial Base}

The expansion of the $r$-dependent transverse-integrated flux over a suitable polynomial basis requires first the definition of the polynomial base then the derivation of the expansion with respect to that base and the pre-computation of scalar products of the Green's function and of the elements of the polynomial base, as shown below.

The polynomial base was derived in a previous project. The desired polynomials must be orthonormal over the radial width of the computational node and must be so with respect to the weight $r$, that is, the polynomials, designated $v_{i}(r)$, must be of order $i$ in $r$ and satisfy the orthonormality condition over the node width in $r$ direction, i.e., over the interval [a,b]:

$$
\left\langle v_{i} \mid v_{j}\right\rangle=\int_{a}^{b} v_{i}(r) v_{j}(r) r d r=\delta_{i j}
$$

The staring point for the construction of the $v_{i}(r)$ polynomials is the natural polynomials base 


$$
u_{n}(r)=r^{n} ; n=0,1,2, \ldots
$$

To this base, the Gram-Schmidt process is applied using the inner product defined in the above equation. Then a recurrence formula for the $v_{i}(r)$ polynomials was constructed:

$$
v_{n}(r)=\alpha_{n}\left\{u_{n}(r)-\sum_{m=0}^{n-1} \beta_{n m} v_{m}(r)\right\}
$$

where $u_{n}(r)$ is defined above, and where

$$
\begin{gathered}
\alpha_{0}=\frac{1}{\sqrt{\gamma_{0}}}, \\
\alpha_{n}^{2}=\frac{1}{\gamma_{2 n}-\sum_{m=0}^{n-1} \beta_{n m}^{2}}, \\
\gamma_{k}=\frac{b^{k+2}-a^{k+2}}{k+2}, \\
\beta_{n 0}=\alpha_{0} \gamma_{n},
\end{gathered}
$$

and

$$
\beta_{n m}=\alpha_{m} \gamma_{m+n}-\alpha_{m} \sum_{l=0}^{m-1} \beta_{m l} \beta_{n l}
$$

\subsection{Flux Expansion}

Using the $v_{n}(r)$ polynomials defined above, the flux in the $l$-th node is expanded according to

$$
z, \theta \overline{\phi_{l}}(r) \cong \sum_{i}^{I} \varphi_{i, l} v_{i}(r)
$$

The expansion coefficients, $\phi_{i l}$, must be determined starting from the flux solution,

$$
{ }^{z, \theta} \overline{\phi_{l}}(r)=\int_{a_{l}}^{b_{l}} G_{l}\left(r, r_{0}\right)^{z, \theta} \overline{S_{l}}\left(r_{0}\right) r_{0} d r_{0}+a_{l} G_{l}\left(r, a_{l}\right)^{z, \theta} \overline{J_{r, l}}\left(a_{l}\right)-b_{l} G_{l}\left(r, b_{l}\right)^{z, \theta} \overline{J_{r, l}}\left(b_{l}\right)
$$

At the end of an iteration of the CMFD method all the elements on the right side of the above equation are known from the solution just computed, except the source terms, which are known from the 
previous iteration. However, together, they provide the necessary information for constructing a representation of the flux at the end of the latest iteration.

Assuming the source terms were expressed as an expansion in the $v_{n}(r)$ polynomials at the end of the previous iteration (in order to be used as sources for the most recent iteration), then the use of the orthonormality conditions of the $v_{n}(r)$ polynomials results in

$$
\begin{aligned}
& \varphi_{j}^{(n)}=\sum_{i=0}^{I}{ }^{z, \theta} \overline{\sigma_{l, i}}{ }^{(n-1)} \int_{a_{l} a_{l}}^{b_{l} b_{l}} G_{l}\left(r, r_{0}\right) v_{i}\left(r_{0}\right) \nu_{i}(r) r_{0} d r_{0} r d r+a_{l}{ }^{z, \theta} \overline{J_{r, l}}\left(a_{l}\right) \int_{a_{l}}^{b_{l}} G_{l}\left(r, a_{l}\right) \nu_{i}(r) r d r \\
& -b_{l}{ }^{z, \theta} \overline{J_{r, l}}\left(b_{l}\right) \int_{a_{l}}^{b_{l}} G_{l}\left(r, b_{l}\right) \nu_{i}(r) r d r
\end{aligned}
$$

In this equation the terms ${ }^{z, \theta}{\overline{\sigma_{l, i}}}^{(n-1)}$ are the expansion coefficients of the source at the end of the (n-1)-th iteration. That source was used in the just completed n-th iteration for which the flux components are computed in the above equation. These components are in turn used to construct the source terms for the $(\mathrm{n}+1)$-th iteration. In the above equation, the interface currents are known from the $\mathrm{n}$-th iteration, except for the source terms that enter into their expression, which are known from the (n1)-th iteration. In the above equation all terms are therefore known and the flux expansion coefficients can be computed. In particular, the integrals involve terms that do not depend on the iterative process and that can, therefore, be pre-computed. The Green's functions that appear in them make analytical precomputing impractical except for the zero order terms, which have already been expressed analytically and coded. In the practical implementation now being carried out, it was first planned to be evaluated all the other integrals to be coded using Gaussian-Legendre quadratures. This planned approach has been revisited. The new approach is discussed below.

\subsection{Green's Matrix and Vector Elements}

In addition to the integrals that appear explicitly in Equation (126) for the flux moments, in the evaluation of the net surface currents two additional such integrals appear. It follows that five integrals are to be evaluated by the best means available, combining analytical expression and, when necessary coded integral evaluations as Gaussian quadratures. This section summarizes the definitions of the needed integral.

\subsubsection{Identification of Required Integrals}

The five integrals that must be evaluated are:

$$
\begin{gathered}
\left\langle j\left|G_{l}\right| i\right\rangle=\int_{a_{l}}^{b_{l}} \int_{a_{l}}^{b_{l}} G_{l}\left(r, r_{0}\right) r r_{0} v_{i}(r) v_{j}\left(r_{0}\right) d r d r_{0}, \\
\left\langle G_{l}^{L}\left(a_{l}\right) \mid i\right\rangle=\int_{a_{l}}^{b_{l}} G_{l}\left(a_{l}, r_{0}\right) r_{0} v_{i}\left(r_{0}\right) d r_{0},
\end{gathered}
$$




$$
\begin{gathered}
\left\langle G_{l}^{L}\left(b_{l}\right) \mid i\right\rangle=\int_{a_{l}}^{b_{l}} G_{l}\left(b_{l}, r_{0}\right) r_{0} v_{i}\left(r_{0}\right) d r_{0}, \\
\left\langle G_{l}^{R}\left(a_{l}\right) \mid i\right\rangle=\int_{a_{l}}^{b_{l}} G_{l}\left(r, a_{l}\right) r v_{i}(r) d r,
\end{gathered}
$$

and

$$
\left\langle G_{l}^{R}\left(b_{l}\right) \mid i\right\rangle=\int_{a_{l}}^{b_{l}} G_{l}\left(r, b_{l}\right) r v_{i}(r) d r \quad .
$$

However, since two pairs of these integrals can be shown to be equal, in practice only three integrals need to be evaluated. This simplification reduces the computational workload accordingly.

The integrals that remain to be evaluated are

$$
\left\langle j\left|G_{l}\right| i\right\rangle=\int_{a_{l}}^{b_{l}} \int_{a_{l}}^{b_{l}} G_{l}\left(r, r_{0}\right) r r_{0} v_{i}(r) v_{j}\left(r_{0}\right) d r d r_{0},
$$

which is termed a "matrix element" or the Green's function and

$$
\left\langle G_{l}^{L}\left(a_{l}\right) \mid i\right\rangle=\int_{a_{l}}^{b_{l}} G_{l}\left(a_{l}, r_{0}\right) r_{0} v_{i}\left(r_{0}\right) d r_{0},
$$

and

$$
\left\langle G_{l}^{L}\left(b_{l}\right) \mid i\right\rangle=\int_{a_{l}}^{b_{l}} G_{l}\left(b_{l}, r_{0}\right) r_{0} v_{i}\left(r_{0}\right) d r_{0} .
$$

The last two integrals are known as vector elements of the Green's function.

Whereas it is easily recognized that all of the above integrals could be evaluated numerically, for example using Gauss-Legendre quadratures, it is also recognized that such an approach would not be the most computationally efficient. Therefore, an attempt was made to evaluate analytically as many of these integrals as possible. It was previously confirmed that the vector elements can be computed exactly for the zero order moments and that they can be computed nearly entirely exactly for the other orders, with only a simple integral remaining that has to be evaluated numerically.

\subsubsection{Evaluation of Vector Elements}

Using the explicit expression for the Green's function, it was shown that the vector elements of the Green's function reduce to: 


$$
\begin{aligned}
& \left\langle G_{l}^{L}\left(a_{l}\right) \mid i\right\rangle=\frac{K_{1}\left(k_{l} b_{l}\right)}{k_{l} a_{l} D_{l}\left[K_{1}\left(k_{l} a_{l}\right) I_{1}\left(k_{l} b_{l}\right)-I_{1}\left(k_{l} a_{l}\right) K_{1}\left(k_{l} b_{l}\right)\right]} \int_{a_{l}}^{b_{l}} I_{0}\left(k_{l} r_{0}\right) v_{i}\left(r_{0}\right) r_{0} d r_{0} \\
& +\frac{I_{1}\left(k_{l} b_{l}\right)}{k_{l} a_{l} D_{l}\left[K_{1}\left(k_{l} a_{l}\right) I_{1}\left(k_{l} b_{l}\right)-I_{1}\left(k_{l} a_{l}\right) K_{1}\left(k_{l} b_{l}\right)\right]} \int_{a_{l}}^{b_{l}} K_{0}\left(k_{l} r_{0}\right) v_{i}\left(r_{0}\right) r_{0} d r_{0}
\end{aligned}
$$

and

$$
\begin{aligned}
\left\langle G_{l}^{L}\left(b_{l}\right) \mid i\right\rangle=\frac{K_{1}\left(k_{l} a_{l}\right)}{k_{l} b_{l} D_{l}[}\left[K_{1}\left(k_{l} a_{l}\right) I_{1}\left(k_{l} b_{l}\right)-I_{1}\left(k_{l} a_{l}\right) K_{1}\left(k_{l} b_{l}\right)\right] & \int_{a_{l}}^{b_{l}} I_{0}\left(k_{l} r_{0}\right) v_{i}\left(r_{0}\right) r_{0} d r_{0} \\
& +\frac{I_{1}\left(k_{l} a_{l}\right)}{k_{l} b_{l} D_{l}\left[K_{1}\left(k_{l} a_{l}\right) I_{1}\left(k_{l} b_{l}\right)-I_{1}\left(k_{l} a_{l}\right) K_{1}\left(k_{l} b_{l}\right)\right]} \int_{a_{l}}^{b_{l}} K_{0}\left(k_{l} r_{0}\right) v_{i}\left(r_{0}\right) r_{0} d r_{0} .
\end{aligned}
$$

It follows, in turn, that the evaluation of all the vector elements of the Green's function reduce to the evaluation of the two simple integrals

$$
\int_{a_{l}}^{b_{l}} I_{0}\left(k_{l} r_{0}\right) v_{i}\left(r_{0}\right) r_{0} d r_{0}
$$

and

$$
\int_{a_{l}}^{b_{l}} K_{0}\left(k_{l} r_{0}\right) v_{i}\left(r_{0}\right) r_{0} d r_{0}
$$

These two integrals have already been evaluated. When the explicit expression for the polynomials $v_{i}\left(r_{0}\right)$ are used, the last two integrals reduce to the integral of the Bessel functions $\mathrm{I}_{0}$ and $\mathrm{K}_{0}$ weighted by powers or $r$. It was shown that all of these integrals can be evaluated nearly entirely in closed analytical forms, except for those corresponding to even power weights, which are also evaluated analytically in closed form save for one additional simple integral,

$$
\int_{k_{l} a_{l}}^{k_{l} b_{l}} I_{0}(x) d x
$$

for the $\mathrm{I}_{0}$ integrals and

$$
\int_{k_{l} a_{l}}^{k_{l} b_{l}} K_{0}(x) d x
$$

for the $\mathrm{K}_{0}$ integrals. Only these two integrals need be evaluated numerically in order to evaluate all of the vector elements of the Green's function for node $l$. 


\subsubsection{Compact form for the Vector Elements}

The vector elements were evaluated per the equations shown above. The last remaining explicit integrals in them are the ones given by the very last two equations above. These last two integrals can be expressed as

$$
\int_{k_{l} a_{l}}^{k_{l} b_{l}} I_{0}(x) d x=k_{l} b_{l} I_{0}\left(k_{l} b_{l}\right)-k_{l} a_{l} I_{0}\left(k_{l} a_{l}\right)+\frac{1}{2} \pi\left[\begin{array}{r}
I_{0}\left(k_{l} b_{l}\right) L_{1}\left(k_{l} b_{l}\right)-I_{1}\left(k_{l} b_{l}\right) L_{0}\left(k_{l} b_{l}\right) \\
-I_{0}\left(k_{l} a_{l}\right) L_{1}\left(k_{l} a_{l}\right)+I_{1}\left(k_{l} a_{l}\right) L_{0}\left(k_{l} a_{l}\right)
\end{array}\right]
$$

where $\mathrm{L}_{\mathrm{i}}(\mathrm{x})$ if the Struve function of order $i$, and

$$
\int_{k_{l} a_{l}}^{k_{l} b_{l}} K_{0}(x) d x=K i_{1}\left(k_{l} a_{l}\right)-K i_{1}\left(k_{l} b_{l}\right)
$$

where $\mathrm{Ki}_{l}(\mathrm{x})$ is the Bickley-Naylor function of order $l$.

By using these expressions, there results an apparently closed form for all the vector elements. However, the reality of the situation is that these seemingly closed forms must actual still be either tabulated on a very fine grid or computed numerically.

\subsubsection{Matrix Elements}

The matrix elements are defined by

$$
\left\langle j\left|G_{l}\right| i\right\rangle=\int_{a_{l}}^{b_{l} b_{l} b_{l}} G_{l}\left(r, r_{0}\right) r r_{0} v_{i}(r) v_{j}\left(r_{0}\right) d r d r_{0}
$$

where the indices $i$ and $j$ must span the values from 0 to $\mathrm{N}$, where $\mathrm{N}$ is the order of the highest polynomial used in the spatial approximation of the source terms.

It was shown that the typical matrix element can be cast in the form

$$
\begin{aligned}
\left\langle j\left|G_{l}\right| i\right\rangle=D^{-1}[ & \left.K_{1}\left(k_{l} a_{l}\right) I_{1}\left(k_{l} b_{l}\right)-K_{1}\left(k_{l} b_{l}\right) I_{1}\left(k_{l} a_{l}\right)\right]^{-1} \\
& \times\left\{K_{1}\left(k_{l} a_{l}\right) K_{1}\left(k_{l} b_{l}\right) S\left(I_{0}, i\right) S\left(I_{0}, j\right)+I_{1}\left(k_{l} a_{l}\right) I_{1}\left(k_{l} b_{l}\right) S\left(K_{0}, i\right) S\left(K_{0}, j\right)\right. \\
& +I_{1}\left(k_{l} a_{l}\right) K_{1}\left(k_{l} b_{l}\right) \int_{a_{l}}^{b_{l}} r v_{i}(r)\left[K_{0}\left(k_{l} r\right) S\left(I_{0}, j ; a_{l}, r\right)+I_{0}\left(k_{l} r\right) S\left(K_{0}, j ; r, b_{l}\right)\right] d r \\
& \left.+K_{1}\left(k_{l} a_{l}\right) I_{1}\left(k_{l} b_{l}\right) \int_{a_{l}}^{b_{l}} r v_{i}(r)\left[I_{0}\left(k_{l} r\right) S\left(K_{0}, j ; a_{l}, r\right)+K_{0}\left(k_{l} r\right) S\left(I_{0}, j ; r, b_{l}\right)\right] d r\right\}
\end{aligned}
$$


where the $\mathrm{S}($ ) functions are similar to the vector elements and are amenable to casting as closed form functions or pseudo-closed form function. The integrals in the above equation can, therefore, be computed numerically using standard techniques.

Although the typical matrix element has been reduced to a form that is amenable to numerical computation, for the sake of computational efficiency work is continuing on expressing these matrix elements in more compact forms and with fewer numerical evaluation, as possible.

\subsection{Status of Generation of Matrix and Vector Elements}

The form show in Equation (134) for the matrix elements has been further manipulated and simplified.

$$
\begin{aligned}
\left\langle j\left|G_{l}\right| i\right\rangle= & \frac{K_{1}\left(k_{l} a_{l}\right) K_{1}\left(k_{l} b_{l}\right)}{D\left[K_{1}\left(k_{l} a_{l}\right) I_{1}\left(k_{l} b_{l}\right)-K_{1}\left(k_{l} b_{l}\right) I_{1}\left(k_{l} a_{l}\right)\right]} S\left(I_{0}, i\right) S\left(I_{0}, j\right) \\
& +\frac{I_{1}\left(k_{l} a_{l}\right) I_{1}\left(k_{l} b_{l}\right)}{D\left[K_{1}\left(k_{l} a_{l}\right) I_{1}\left(k_{l} b_{l}\right)-K_{1}\left(k_{l} b_{l}\right) I_{1}\left(k_{l} a_{l}\right)\right]} S\left(K_{0}, i\right) S\left(K_{0}, j\right) \\
& +\frac{1}{2} \frac{I_{1}\left(k_{l} a_{l}\right) K_{1}\left(k_{l} b_{l}\right)+K_{1}\left(k_{l} a_{l}\right) I_{1}\left(k_{l} b_{l}\right)}{D\left[K_{1}\left(k_{l} a_{l}\right) I_{1}\left(k_{l} b_{l}\right)-K_{1}\left(k_{l} b_{l}\right) I_{1}\left(k_{l} a_{l}\right)\right]}\left[S\left(I_{0}, i\right) S\left(K_{0}, j\right)+S\left(K_{0}, i\right) S\left(I_{0}, j\right)\right] \\
& +\frac{1}{2} \int_{a_{l}}^{b_{l}} r v_{i}(r) K_{0}\left(k_{l} r\right)\left[S\left(I_{0}, j ; r, b_{l}\right)-S\left(I_{0}, j ; a_{l}, r\right)\right] d r \\
& +\frac{1}{2} \int_{a_{l}}^{b_{l}} r v_{i}(r) I_{0}\left(k_{l} r\right)\left[S\left(K_{0}, j ; a_{l}, r\right)-S\left(K_{0}, j ; r, b_{l}\right)\right] d r
\end{aligned}
$$

In the above equation, the complete $S$ functions, $S\left(I_{0}, i\right), S\left(I_{0, j}\right), S\left(K_{0}, i\right)$, and $S\left(K_{0, j}\right)$ are known either in fully closed form or nearly closed form (where a single integral evaluation remains to be carried out explicitly, either numerically or through tabulated interpolation). The only terms that require further manipulation to yield simpler and easier to evaluate terms are the two integrals. The incomplete $\mathrm{S}$ functions that enter into the definition of these equations and the integrals of these incomplete $\mathrm{S}$ functions are currently being evaluated. The evaluation of the $\mathrm{S}$ function has now been completed and about $1 / 3$ of the integrals have also been completed. The effort is to continue into the next fiscal year. 


\section{CONCLUSIONS}

A new hybrid method using response functions for the treatment of control rods and other nonmultiplying zones within reactors has been developed and implemented. Response functions to test the implementation were derived from the highly accurate nodal Green's function solutions and were used to test the implementation within the CYNOD code. Discontinuity factors were also implemented within the hybrid CYNOD code. The results display close agreement between the solutions generated using the hybrid CYNOD code and those obtained using the standard CYNOD code. The test response functions and discontinuity factors were generated from a fine mesh calculation. The coarse mesh CYNOD results are better (i.e., more accurate) when discontinuity factors are used. Overall, the results obtained show that the algorithm has been correctly implemented. The testing provides verification of the model fidelity and implementation, but not validation of the overall code. Testing of the validity of the approach for modeling control rods remains to be carried out using actual response functions derived from neutron transport calculations.

The derivation of expansions for the radial flux and radial source terms has progressed substantially. A rational framework for the approximation of the source has been devised and the resulting matrix elements and vector elements for the Green's function have been fully formulated. These vector and matrix elements are being evaluated explicitly and about $1 / 3$ are fully formulated. 


\section{REFERENCES}

1. Reitsma, F. and Naidoo, D., "Evaluating the control rod modelling approach used in the South African PBMR: comparison of VSOP calculations with ASTRA experiments", Nuclear Engineering and Design, Volume 222, Issues 2-3, June 2003, Pages 147-159. This paper cites the original source: Scherer, W. and Neff, H.J., "Determination of Equivalent Cross Sections for Representation of Control Rod Regions in Diffusion Calculations", Jul 1311, (1976)

2. Fen, V., et al., "Modeling of Neutron Absorbers in High Temperature Reactors by Combined Transport-Diffusion Methods," Jul 2573, (1992)

3. Ougouag, A. M., "A Coarse-Mesh Nodal Method for Multigroup Multidimensional Neutron Diffusion Computations", M.S. Thesis, University of Illinois, 1981.

4. Rajic, H.L., and Ougouag A. M., "ILLICO: A Nodal Neutron Diffusion Method for Modern Computer Architectures," Nuclear Science and Engineering, 103, 4 392-408 (1989).

5. M. Ougouag, "Derivation of Coarse-Mesh Finite Difference Equations for Cylindrical Geometry Nodal Neutron Diffusion for the PEBBED and CYNOD codes," unpublished Idaho National Laboratory notes, 2001-2004.

6. Hiruta, H., et al., 2008, "CYNOD: A Neutronics Code for Pebble Bed Modular Reactor Coupled Transient Analysis "paper HTR2008-58255 in Proceedings of the 4th International Topical Meeting on High Temperature Reactor Technology - HTR2008, September 28-October 1, 2008, Washington, DC USA, on CD-ROM.

7. Ougouag, A. M., et al., 2006, "Development of a Method for Neutronics Treatment of Non-multiplying Zones in Pebble Bed Reactors," Proceedings of the 3rd International Topical Meeting on High Temperature Reactor Technology, HTR2006, Johannesburg, South Africa, 2006.

8. “OECD/NEA/NSC PBMR Coupled Neutronic/Thermal Hydraulics Transient Benchmark: The PBMR-400 Core Design,” Draft 07 (2005) 